\title{
Likelihood analysis of the flavour anomalies and $g-2$ in the general two Higgs doublet model
}

Peter Athron, ${ }^{a, b}$ Csaba Balazs, ${ }^{b}$ Tomás E. Gonzalo, ${ }^{c}$ Douglas Jacob, ${ }^{b}$ Farvah Mahmoudi ${ }^{d, e}$ and Cristian Sierra ${ }^{b}$

${ }^{a}$ Department of Physics and Institute of Theoretical Physics, Nanjing Normal University, Wenyuan Road, Nanjing, Jiangsu, 210023, China

${ }^{b}$ School of Physics and Astronomy, Monash University,

Wellington Road, Clayton, VIC 3800, Australia

${ }^{c}$ Institute for Theoretical Particle Physics and Cosmology (TTK), RWTH Aachen, Sommerfeldstrasse 12, 52074 Aachen, Germany

${ }^{d}$ Université de Lyon, Université Claude Bernard Lyon 1, CNRS/IN2P3,

Institut de Physique des 2 Infinis de Lyon, UMR 5822,

69622 Villeurbanne, France

e Theoretical Physics Department, CERN,

CH-1211 Geneva 23, Switzerland

E-mail: peter.athron@coepp.org.au, csaba.balazs@monash.edu,

tomas.gonzalo@monash.edu, douglas.jacob@monash.edu, mahmoudi@in2p3.fr, cristian.sierra@monash.edu 
ABSTRACT: We present a likelihood analysis of the general two Higgs doublet model, using the most important currently measured flavour observables, in view of the anomalies in charged current tree-level and neutral current one-loop rare decays of $B$ mesons in $b \rightarrow c l \bar{\nu}$ and $b \rightarrow s \mu^{+} \mu^{-}$transitions, respectively. We corroborate that the model explains the latter and it is able to simultaneously fit the experimental values of the $R(D)$ charged current ratio at $1 \sigma$, but it can not accommodate the $D^{*}$ charmed meson observables $R\left(D^{*}\right)$ and $F_{L}\left(D^{*}\right)$. We find that the fitted values for the angular observables in $b \rightarrow s \mu^{+} \mu^{-}$transitions exhibit better agreement with the general two Higgs double model in comparison to the SM. We also make predictions for future collider observables $\operatorname{BR}(t \rightarrow c h), \operatorname{BR}(h \rightarrow b s)$, $\mathrm{BR}(h \rightarrow \tau \mu), \mathrm{BR}\left(B_{s} \rightarrow \tau^{+} \tau^{-}\right), \mathrm{BR}\left(B^{+} \rightarrow K^{+} \tau^{+} \tau^{-}\right)$and the flavour violating decays of the $\tau$ lepton, $\operatorname{BR}(\tau \rightarrow 3 \mu)$ and $\operatorname{BR}(\tau \rightarrow \mu \gamma)$. The model predicts values of $\operatorname{BR}(t \rightarrow c h)$, $\mathrm{BR}\left(B_{s} \rightarrow \tau^{+} \tau^{-}\right)$and $\operatorname{BR}\left(B^{+} \rightarrow K^{+} \tau^{+} \tau^{-}\right)$that are out of reach of future experiments, but its predictions for $\mathrm{BR}(h \rightarrow b s)$ and $\mathrm{BR}(h \rightarrow \tau \mu)$ are within the future sensitivity of the HL-LHC or the ILC. We also find that the predictions for the $\tau \rightarrow 3 \mu$ and $\tau \rightarrow \mu \gamma$ decays are well within the projected limits of the Belle II experiment. Finally, using the latest measurement of the Fermilab Muon $g-2$ Collaboration, we performed a simultaneous fit to $\Delta a_{\mu}$ constrained by the charged anomalies, finding solutions at the $1 \sigma$ level. Once the neutral anomalies are included, however, a simultaneous explanation is unfeasible.

KEYwords: Phenomenological Models

ARXIV EPRINT: 2111.10464 


\section{Contents}

1 Introduction 1

2 GTHDM 3

2.1 Higgs potential 4

2.2 Yukawa Lagrangian 5

3 Effective Hamiltonians for flavour changing transitions $\quad 6$

$3.1 b \rightarrow s \ell^{+} \ell^{-}$transitions

3.1.1 Penguins and boxes computation $\quad 8$

$\begin{array}{lll}3.1 .2 & \text { Summary of contributions } & 10\end{array}$

$3.2 \quad b \rightarrow c \ell \bar{\nu}$ semileptonic transitions $\quad 11$

4 Observables 12

$\begin{array}{lll}4.1 & \text { FCNCs and } B \text { rare decays } & 12\end{array}$

$\begin{array}{lll}4.2 & \text { FCCCs observables } & 14\end{array}$

$\begin{array}{lll}4.3 & \text { Leptonic decays of mesons } & 16\end{array}$

$\begin{array}{ll}4.4 \text { Leptonic observables } & 17\end{array}$

5 Results $\quad 21$

5.1 Parameter space 21

5.2 Neutral and charged anomalies 24

$\begin{array}{ll}5.3 \text { Anomalous }(g-2)_{\mu} & 28\end{array}$

$\begin{array}{lll}5.4 & \text { Projections for future and planned experiments } & 29\end{array}$

6 Conclusions and outlook 31

A Gauge dependent term $\quad 32$

B Loop functions and vertex couplings 33

$\begin{array}{ll}\text { C Auxiliary scanning method } & 37\end{array}$

\section{Introduction}

The Standard Model (SM) of particle physics contains three fermion families which acquire mass by means of the interaction with the Higgs boson. The two Higgs doublet model $(2 \mathrm{HDM})$ is one of the simplest ways to extend the Higgs sector, which is the least constrained sector of the Standard Model. Two Higgs doublets also appear in many more 
elaborate extensions of the SM that are based on fundamental principles, such as supersymmetry (see e.g. [1]), the Peccei-Quinn symmetry [2, 3] or grand unified theories (see [4] for a recent review). Two Higgs doublet models are also motivated from electroweak baryogenesis studies, where it has been shown that contributions coming from the new physical Higgs bosons to the effective Higgs potential can strengthen the phase transition and in addition introduce new sources of charge-parity $(\mathrm{CP})$ violation, from both fermion and scalar sectors [5-13]. As a result, the 2HDM is one of the most popular SM extensions and has been frequently used as a benchmark for phenomenological studies (see e.g. [14] for a review of 2HDM studies). Furthermore, the presence of another doublet can contribute to resolving anomalies in lepton flavour universality observables $[15,16]$ and muon g-2 [17-46], while scenarios where the extra doublet is "inert" can also explain dark matter [47-59].

The new interactions between the SM fermions and the physical states arising from the introduction of a second Higgs doublet imply a richer phenomenology than the SM. This is further enhanced by the new free parameters and couplings in the general two Higgs doublet model (GTHDM), also known as type-III 2HDM [60]. Physical effects such as CP violation, scalar mixing and flavour changing transitions are expected [61], allowing for signatures to be observed in particle colliders. One of the most interesting experimental consequences of the flavour changing currents present in the GTHDM is lepton flavour universality (LFU) violation. Experimental measurements of LFU violation come from flavour changing charged currents (FCCCs), such as those in $B$ meson decays, and flavour changing neutral currents (FCNCs), for instance in kaon decays. The observed deviations from the SM in the measurements of FCCCs (around 3.1 $\sigma$ from the SM [62]) and FCNCs (close to a combined $6 \sigma$ deviation, see for example [63-65]), hint at the existence of new physics (NP) contributions and thus serve as a clear motivation for the study of NP models capable of explaining the anomalies.

It has indeed been shown that the GTHDM is able to explain the charged anomalies at $2 \sigma[15,16,66,67]$. Similar analyses for the neutral anomalies have also been presented previously [15, 68-70], finding solutions at the $2 \sigma$ level and up to the $1 \sigma$ level including right-handed neutrinos [70]. Nevertheless, the majority of these studies have only explored solutions in restricted regions of the parameter space, with a lack of discussion of the role of (marginally) statistically preferred regions, and often considering the $b \rightarrow$ sll observables from model independent global fits $[15,70]$. Statistically rigorous explorations of the parameter space of the model contrasted directly to experimental constraints have rarely been performed, and even those were focused exclusively on interactions in the quark sector [71].

Furthermore, the longstanding discrepancy between the experimentally measured and SM predicted values of the anomalous magnetic moment of the muon $a_{\mu}$ has recently been brought back to the spotlight with the new measurement by the Muon g-2 experiment at Fermilab [72]. The latest experimental value, taking into account the measurements at both Brookhaven National Laboratory and Fermilab, is $a_{\mu}^{\text {Exp }}=116592061 \pm 41 \times 10^{-11}$. Compared to the theoretical prediction in the SM from the recent Muon $g-2$ Theory Initiative White Paper, $a_{\mu}^{\mathrm{SM}}=116591810 \pm 43 \times 10^{-11}$ [73], building on the extensive work examining the various SM contributions in [74-93], the measured value differs from the SM prediction by $\Delta a_{\mu}=2.51 \pm 59 \times 10^{-9}$, corresponding to a discrepancy of $4.2 \sigma$. Models with 
a second Higgs doublet have been studied extensively in the literature as sources to explain this deviation [17-46]. However, no simultaneous global fit of the flavour anomalies and $a_{\mu}$ in the GTHDM has been attempted giving a proper statistical insight into the whole parameter space.

Therefore, in this paper we present a frequentist inspired likelihood analysis for the GTHDM, simultaneously including the FCCC observables, both $b \rightarrow s \mu^{+} \mu^{-}$transitions and the muon anomalous magnetic moment, along with other flavour observables. We perform a global fit of all constraints using the inference package GAMBIT, the Global And Modular Beyond-the-Standard-Model Inference Tool [94, 95]. GAMBIT is a powerful software framework capable of performing statistical inference studies using constraints from collider [96], dark matter [97], flavour [98] and neutrino [99] physics, as well as cosmology [100]. It has already been used for detailed statistical analyses of a variety of beyond the Standard Model (BSM) models, including supersymmetry [101-103], scalar singlet DM [104-108], axion and axion-like particles [109, 110], and neutrinos [99, 111], as well as an initial analysis of the 2HDM [112]. Our work enhances the FlavBit [98] and PrecisionBit [113] modules of GAMBIT to support the GTHDM. We also make use of various external codes: Superlso 4.1 [114-117] for computing flavour observables, the 2HDMC 1.8 package [118] for precision electroweak constraints, the HEPLike package [119] which provides likelihoods for the neutral anomaly related observables, and the differential evolution sampler Diver 1.0.4 [120].

The paper is organised as follows. In section 2 we present the Higgs and Yukawa sectors along the theoretical bounds for their parameters. In section 3 we define the effective Hamiltonian and the Wilson coefficients (WCs) for $b \rightarrow s \mu^{+} \mu^{-}$transitions. Then, in section 4 we list the observables to be used in our scans. Following this, our results from the global fit and predictions for future experiments in colliders are discussed in section 5 . Finally, we summarise our conclusions in section 6 .

\section{GTHDM}

The GTHDM has been actively investigated in both its scalar and Yukawa sectors. These can be written in three different ways, namely in the generic, Higgs and physical bases, all of them related via basis transformations [121]. Particularly, with respect to the Yukawa sector, in the past theorists imposed discrete symmetries to avoid flavour changing transitions, the most popular being the $\mathbb{Z}_{2}$ symmetry in the type-II $2 \mathrm{HDM}[122,123]$. However, it has been shown that there is no fundamental reason for forbidding flavour changing couplings [124]: if the mixing angle is small, the non-observation of several tree level flavour changing transitions can be explained by the alignment phenomenon. This, and a suppression inversely proportional to the mass of the heavy Higgses in the tree level amplitudes, could suppress the effects coming from the off-diagonal Yukawa couplings, without invoking the so called natural flavour conservation (NFC) condition [122].

Here we review the Higgs potential and the Yukawa Lagrangian of the model as well as the relevant theoretical constraints coming from stability, unitarity and perturbativity at leading order (LO). We also make use of the precision electroweak constraints from the 
oblique parameters. For a more comprehensive review of the model the reader is referred to $[14,125-127]$.

\subsection{Higgs potential}

The most general renormalizable scalar potential in the GTHDM is commonly written as $[14,128]$

$$
\begin{aligned}
V\left(\Phi_{1}, \Phi_{2}\right)= & m_{11}^{2}\left(\Phi_{1}^{\dagger} \Phi_{1}\right)+m_{22}^{2}\left(\Phi_{2}^{\dagger} \Phi_{2}\right)-m_{12}^{2}\left(\Phi_{1}^{\dagger} \Phi_{2}+\Phi_{2}^{\dagger} \Phi_{1}\right) \\
& +\frac{1}{2} \lambda_{1}\left(\Phi_{1}^{\dagger} \Phi_{1}\right)^{2}+\frac{1}{2} \lambda_{2}\left(\Phi_{2}^{\dagger} \Phi_{2}\right)^{2}+\lambda_{3}\left(\Phi_{1}^{\dagger} \Phi_{1}\right)\left(\Phi_{2}^{\dagger} \Phi_{2}\right)+\lambda_{4}\left(\Phi_{1}^{\dagger} \Phi_{2}\right)\left(\Phi_{2}^{\dagger} \Phi_{1}\right) \\
& +\left(\frac{1}{2} \lambda_{5}\left(\Phi_{1}^{\dagger} \Phi_{2}\right)^{2}+\left(\lambda_{6}\left(\Phi_{1}^{\dagger} \Phi_{1}\right)+\lambda_{7}\left(\Phi_{2}^{\dagger} \Phi_{2}\right)\right)\left(\Phi_{1}^{\dagger} \Phi_{2}\right)+\text { h.c. }\right)
\end{aligned}
$$

where the two scalar doublets are given by

$$
\Phi_{i}=\left(\begin{array}{c}
\phi_{i}^{+} \\
\frac{1}{\sqrt{2}}\left(v_{i}+\rho_{i}+i \eta_{i}\right)
\end{array}\right), \quad i=1,2,
$$

with $v_{i}$ the vacuum expectation values (VEV) of the fields, while linear combinations of the fields $\rho_{i}, \eta_{i}$ and $\phi_{i}^{ \pm}$form mass eigenstates:

$$
\left(\begin{array}{c}
G_{Z} \\
A
\end{array}\right)=R_{\beta}\left(\begin{array}{c}
\eta_{1} \\
\eta_{2}
\end{array}\right), \quad\left(\begin{array}{c}
G_{W^{ \pm}} \\
H^{ \pm}
\end{array}\right)=R_{\beta}\left(\begin{array}{c}
\phi_{1}^{ \pm} \\
\phi_{2}^{ \pm}
\end{array}\right), \quad\left(\begin{array}{c}
H \\
h
\end{array}\right)=R_{\alpha}\left(\begin{array}{c}
\rho_{1} \\
\rho_{2}
\end{array}\right),
$$

where the fields $\phi_{i}^{+}$are charged complex scalars. From the eight degrees of freedom, three of them $\left(G_{W^{ \pm}}\right.$and $\left.G_{Z}\right)$ get absorbed by the longitudinal components of the vector bosons. The remaining five make up the new particle spectrum of the model, namely, $h$ and $H$ are physical CP-even states, $A$ is a CP-odd state and $H^{ \pm}$are two charged Higgs bosons. The rotation matrices are defined according to

$$
R_{\theta}=\left(\begin{array}{cc}
\cos \theta & \sin \theta \\
-\sin \theta & \cos \theta
\end{array}\right) \text {. }
$$

In this work, we assume a $\mathrm{CP}$ conserving scalar sector, which implies all the parameters in eq. (2.1) to be real [128]. Additionally, for simplicity, we set $\lambda_{6}=\lambda_{7}=0$. In particular, for this choice of the quartic couplings, the necessary and sufficient conditions to ensure positivity of the potential along all directions are given by [14, 128]

$$
\begin{aligned}
\lambda_{1} & \geq 0, & \lambda_{2} & \geq 0, \\
\lambda_{3} & \geq-\sqrt{\lambda_{1} \lambda_{2}}, & \lambda_{3}+\lambda_{4}-\left|\lambda_{5}\right| & \geq-\sqrt{\lambda_{1} \lambda_{2}},
\end{aligned}
$$

whereas the tree level unitarity of the couplings imposes [14]

$$
\left|a_{ \pm}\right|,\left|b_{ \pm}\right|,\left|c_{ \pm}\right|,\left|d_{ \pm}\right|,\left|e_{ \pm}\right|,\left|f_{ \pm}\right|<8 \pi
$$


where

$$
\begin{aligned}
a_{ \pm} & =\frac{3}{2}\left(\lambda_{1}+\lambda_{2}\right) \pm \sqrt{\frac{9}{4}\left(\lambda_{1}-\lambda_{2}\right)^{2}+\left(2 \lambda_{3}+\lambda_{4}\right)^{2}} \\
b_{ \pm} & =\frac{1}{2}\left(\lambda_{1}+\lambda_{2}\right) \pm \frac{1}{2}, \sqrt{\left(\lambda_{1}-\lambda_{2}\right)^{2}+4 \lambda_{4}^{2}}, \\
c_{ \pm} & =\frac{1}{2}\left(\lambda_{1}+\lambda_{2}\right) \pm \frac{1}{2} \sqrt{\left(\lambda_{1}-\lambda_{2}\right)^{2}+4 \lambda_{5}^{2}}, \\
d_{ \pm} & =\lambda_{3}+2 \lambda_{4} \pm 3 \lambda_{5}, \\
e_{ \pm} & =\lambda_{3} \pm \lambda_{5}, \\
f_{ \pm} & =\lambda_{3} \pm \lambda_{4} .
\end{aligned}
$$

Following [71, 125] we also include the oblique parameters $S, T$ and $U$, which parametrise radiative corrections to electroweak gauge boson propagators. In this study we computed these oblique parameters with the 2 HDMC package and these are contrasted with the most probable values inferred from experimental data, as found by the Gfitter group [129]

$$
S=0.05 \pm 0.11, \quad T=0.09 \pm 0.13, \quad U=0.01 \pm 0.11,
$$

with correlations given by

$$
\Sigma=\left(\begin{array}{ccc}
1.0 & 0.9 & -0.59 \\
0.9 & 1.0 & -0.83 \\
-0.59 & -0.83 & 1.0
\end{array}\right)
$$

\subsection{Yukawa Lagrangian}

The most general Yukawa Lagrangian in the generic scalar basis $\left\{\Phi_{1}, \Phi_{2}\right\}$ reads [71]:

$$
-\mathcal{L}_{\text {Yukawa }}=\bar{Q}^{0}\left(Y_{1}^{u} \tilde{\Phi}_{1}+Y_{2}^{u} \tilde{\Phi}_{2}\right) u_{\mathrm{R}}^{0}+\bar{Q}^{0}\left(Y_{1}^{d} \Phi_{1}+Y_{2}^{d} \Phi_{2}\right) d_{\mathrm{R}}^{0}+\bar{L}^{0}\left(Y_{1}^{l} \Phi_{1}+Y_{2}^{l} \Phi_{2}\right) l_{\mathrm{R}}^{0}+\text { h.c. },
$$

where the superscript " 0 " notation refers to the flavour eigenstates, and $\tilde{\Phi}_{j}=i \sigma_{2} \Phi_{j}^{\dagger}$. The fermion mass matrices are given by

$$
M_{f}=\frac{1}{\sqrt{2}}\left(v_{1} Y_{1}^{f}+v_{2} Y_{2}^{f}\right), \quad f=u, d, l .
$$

Notice that this matrices need to be diagonalized. This can be done through a bi-unitary transformation

$$
\bar{M}_{f}=V_{f L}^{\dagger} M_{f} V_{f R},
$$

where the fact that $M_{f}$ is Hermitian implies that $V_{f L}=V_{f R}$, and the mass eigenstates for the fermions are given by

$$
u=V_{u}^{\dagger} u^{0}, \quad d=V_{d}^{\dagger} d^{0}, \quad l=V_{l}^{\dagger} l^{0} .
$$

Then, eq. (2.17) takes the form

$$
\bar{M}_{f}=\frac{1}{\sqrt{2}}\left(v_{1} \tilde{Y}_{1}^{f}+v_{2} \tilde{Y}_{2}^{f}\right),
$$


where $\tilde{Y}_{i}^{f}=V_{f L}^{\dagger} Y_{i}^{f} V_{f R}$, though each Yukawa matrix is not diagonalized by this transformation. For this reason we shall drop the tilde from now on. Solving for $Y_{1}^{f}$ we have

$$
Y_{1, b a}^{f}=\frac{\sqrt{2}}{v \cos \beta} \bar{M}_{f, b a}-\tan \beta Y_{2, b a}^{f} .
$$

Using the expressions above we can write the Yukawa Lagrangian in the mass basis as ${ }^{1}$

$$
\begin{aligned}
-\mathcal{L}_{\text {Yukawa }}= & \bar{u}_{b}\left(V_{b c} \xi_{c a}^{d} P_{R}-V_{c a} \xi_{c b}^{u *} P_{L}\right) d_{a} H^{+}+\bar{\nu}_{b} \xi_{b a}^{l} P_{R} l_{a} H^{+}+\text {h.c. } \\
& +\sum_{f=u, d, e} \sum_{\phi=h, H, A} \bar{f}_{b} \Gamma_{\phi b a}^{f} P_{R} f_{a} \phi+\text { h.c. },
\end{aligned}
$$

where $a, b=1,2,3$ and

$$
\begin{gathered}
\xi_{b a}^{f} \equiv \frac{Y_{2, b a}^{f}}{\cos \beta}-\frac{\sqrt{2} \tan \beta \bar{M}_{f, b a}}{v}, \\
\Gamma_{h b a}^{f} \equiv \frac{\bar{M}_{f, b a}}{v} s_{\beta-\alpha}+\frac{1}{\sqrt{2}} \xi_{b a}^{f} c_{\beta-\alpha}, \\
\Gamma_{H b a}^{f} \equiv \frac{\bar{M}_{f, b a}}{v} c_{\beta-\alpha}-\frac{1}{\sqrt{2}} \xi_{b a}^{f} s_{\beta-\alpha}, \\
\Gamma_{A b a}^{f} \equiv \begin{cases}-\frac{i}{\sqrt{2}} \xi_{b a}^{f} & \text { if } f=u, \\
\frac{i}{\sqrt{2}} \xi_{b a}^{f} & \text { if } f=d, l .\end{cases}
\end{gathered}
$$

At first, the total number of new complex Yukawa couplings to consider is 54. Considering only their real parts and the ansatz

$$
\xi^{u}=\left(\begin{array}{ccc}
0 & 0 & 0 \\
0 & \xi_{c c}^{u} & \xi_{c t}^{u} \\
0 & \xi_{t c}^{u} & \xi_{t t}^{u}
\end{array}\right), \quad \xi^{d}=\left(\begin{array}{ccc}
0 & 0 & 0 \\
0 & \xi_{s s}^{d} & \xi_{s b}^{d} \\
0 & \xi_{b s}^{d} & \xi_{b b}^{d}
\end{array}\right), \quad \xi^{l}=\left(\begin{array}{ccc}
0 & 0 & 0 \\
0 & \xi_{\mu \mu}^{l} & \xi_{\mu \tau}^{l} \\
0 & \xi_{\tau \mu}^{l} & \xi_{\tau \tau}^{l}
\end{array}\right),
$$

we get only 12 Yukawa parameters (i.e., ignoring $3 \rightarrow 1$ and $2 \rightarrow 1$ generation transitions). Here, the $\xi^{u}$ matrix has been previously considered to be asymmetric from $B_{s}-\bar{B}_{s}$ oscillations constraints at one loop level and for heavy Higgs masses of order $\lesssim 700 \mathrm{GeV}[130,131]$. However, since we are approaching the dominant contribution process at LO and we are exploring masses in the range $[0.5,4.0] \mathrm{TeV}$ as in [71], we will consider only the symmetric case, i.e., $\xi_{t c}^{u}=\xi_{c t}^{u}$. Hence, assuming the remaining $\xi^{d}$ and $\xi^{l}$ matrices to be symmetric as well, the total number of parameters to scan over is reduced by 3 .

\section{Effective Hamiltonians for flavour changing transitions}

Most of the relevant flavour observables that we consider in this work arise from processes with either suppressed or negligible contributions from SM particles. Hence, these processes

\footnotetext{
${ }^{1}$ This Yukawa Lagrangian differs from the one defined in eq. (2.3) in [70] by an overall factor of $\sqrt{2}$.
} 
are often dominated by BSM contributions, which can be generated by a large variety of UV complete theories. It is often convenient to study these transitions using the, modelagnostic, effective Hamiltonian approach, where transition operators are decomposed using the Operator Product Expansion (OPE) into a collection of simple, low-energy, operators. Associated with each of these operators comes a WC, which encodes the knowledge of the high-energy theory. Even for complete high-energy theories, as it is our case, it is extremely useful to work with the effective Hamiltonian, since one can easily compute most observables of interest in terms of a small set of WCs. In fact, there are only two independent flavour changing transitions that give rise to the majority of the studied observables, and these are the neutral $b \rightarrow s \ell^{+} \ell^{-}$transition and the charged $b \rightarrow c \ell \bar{\nu}$ transition. In this section we write down the effective Hamiltonian for both of these transitions and provide expressions for the BSM contributions to the WCs that arise in our model. ${ }^{2}$

\section{$3.1 \quad b \rightarrow s \ell^{+} \ell^{-}$transitions}

The effective Hamiltonian responsible for $b \rightarrow s \ell^{+} \ell^{-}$transitions can be written as

$$
\mathcal{H}_{\mathrm{eff}}=-\frac{4 G_{F}}{\sqrt{2}} V_{t b} V_{t s}^{*}\left[\sum_{i=S, P} C_{i}(\mu) \mathcal{O}_{i}+C_{i}^{\prime}(\mu) \mathcal{O}_{i}^{\prime}+\sum_{i=7}^{10} C_{i}(\mu) \mathcal{O}_{i}+C_{i}^{\prime}(\mu) \mathcal{O}_{i}^{\prime}\right],
$$

where $\mu$ is the energy scale at which the WCs are defined, and

$$
\begin{aligned}
\mathcal{O}_{9} & =\frac{e^{2}}{16 \pi^{2}}\left(\bar{s} \gamma_{\mu} P_{L} b\right)\left(\bar{\ell} \gamma^{\mu} \ell\right), & \mathcal{O}_{10} & =\frac{e^{2}}{16 \pi^{2}}\left(\bar{s} \gamma_{\mu} P_{L} b\right)\left(\bar{\ell} \gamma^{\mu} \gamma_{5} \ell\right), \\
\mathcal{O}_{S} & =\frac{e^{2}}{16 \pi^{2}} m_{b}\left(\bar{s} P_{R} b\right)(\bar{\ell} \ell), & \mathcal{O}_{P} & =\frac{e^{2}}{16 \pi^{2}} m_{b}\left(\bar{s} P_{R} b\right)\left(\bar{\ell} \gamma_{5} \ell\right), \\
\mathcal{O}_{7} & =\frac{e}{16 \pi^{2}} m_{b}\left(\bar{s} \sigma^{\mu \nu} P_{R} b\right) F_{\mu \nu}, & \mathcal{O}_{8} & =\frac{g}{16 \pi^{2}} m_{b} \bar{s} \sigma^{\mu \nu} T^{a} P_{R} b G_{\mu \nu}^{a},
\end{aligned}
$$

are the FCNC local operators encoding the low-energy description of the high energy physics that has been integrated out. The prime operators are obtained by the replacement $P_{R(L)} \rightarrow P_{L(R)}$. The WCs can be written as

$$
C_{i}=C_{i}^{\mathrm{SM}}+\Delta C_{i}
$$

where $C_{i}^{\mathrm{SM}}$ is the SM contribution to the $i$ th WC and $\Delta C_{i}$ is the NP contribution, a prediction of the GTHDM model. The SM contribution to the scalar WCs, $C_{S, P}^{(\prime)}$, is negligible, whereas for $C_{7-10}$ we have

$$
\operatorname{Re}\left(C_{7,8,9,10}^{\mathrm{SM}}\right)=-0.297,-0.16,4.22,-4.06,
$$

as computed with Superlso. We evaluate the NP scalar and pseudoscalar coefficients $\Delta C_{S, P}^{(\prime)}$ at tree level, which is the LO contribution from the GTHDM [70]. Henceforth we will use the scalar and pseudoscalar coefficients in the basis defined in Superlso, i.e., $C_{Q_{1}, Q_{2}}^{\left({ }^{\prime}\right)}=$ $m_{b(s)} C_{S, P}^{\left({ }^{\prime}\right)}$. The remaining coefficients, $\Delta C_{7,8,9,10}$ first appear at one loop level and we

\footnotetext{
${ }^{2}$ These BSM new contributions for $b \rightarrow s \ell^{+} \ell^{-}$and $b \rightarrow c \ell \bar{\nu}$ transitions were included in our local version of FlavBit and might appear in a future release.
} 

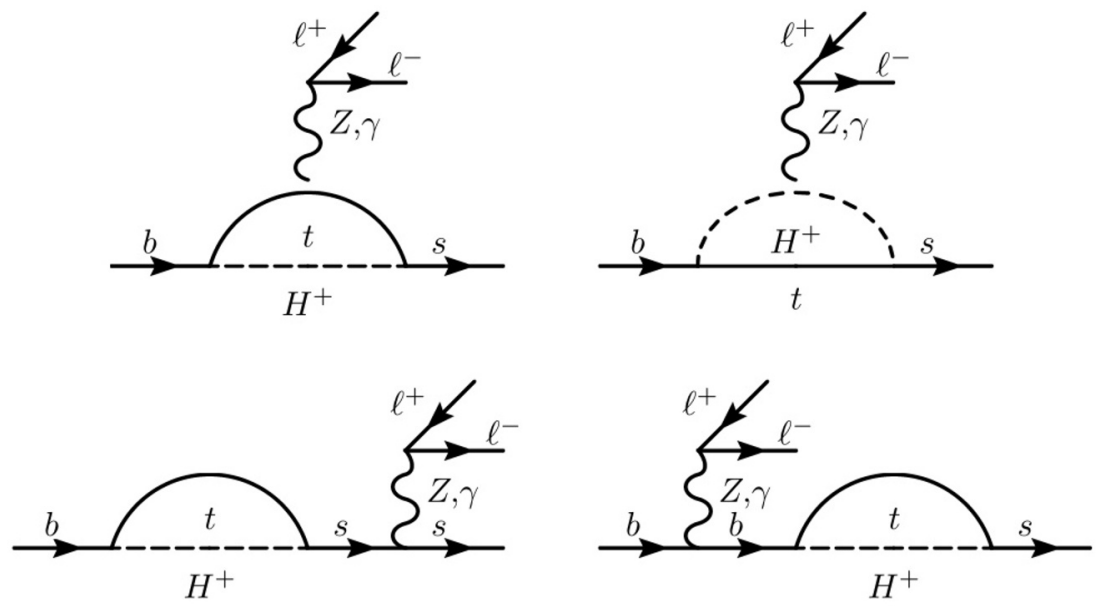

Figure 1. Penguin diagrams for $b \rightarrow s \ell^{+} \ell^{-}$transitions.

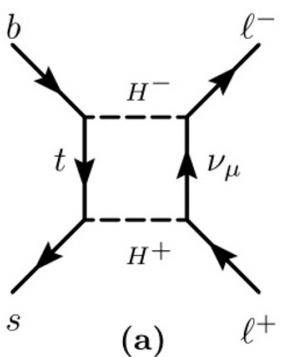

(a)

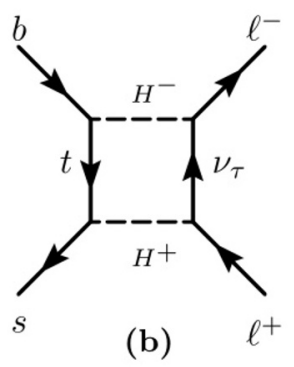

(b)

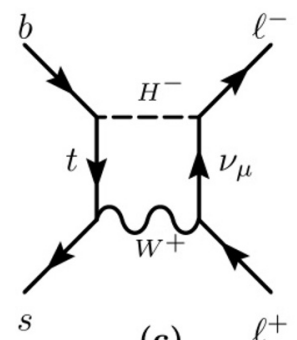

(c)

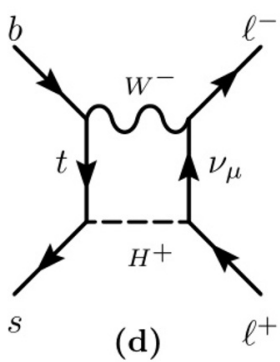

(d)

Figure 2. Box diagrams for $b \rightarrow s \ell^{+} \ell^{-}$transitions.

therefore include the one-loop BSM contributions to these in our analysis. These one-loop corrections can be split by contribution as follows,

$$
\begin{aligned}
\Delta C_{7,8} & =C_{7,8}^{\gamma, g}, \\
\Delta C_{9} & =C_{9}^{\gamma}+C_{9}^{Z}+C_{9}^{\text {box }}, \\
\Delta C_{10} & =C_{10}^{Z}+C_{10}^{\text {box }} .
\end{aligned}
$$

where $C_{9,10}^{Z}$ and $C_{7,9}^{\gamma}$ come from the $Z$ and $\gamma$ penguins, respectively (figure 1 ), and $C_{9,10}^{\text {box }}$ are contributions from box diagrams, (figure 2). At this level, the $\Delta C_{9}^{\prime}$ and $\Delta C_{10}^{\prime}$ coefficients are suppressed as $m_{b} / m_{t}$ with respect to their non-prime counterparts. However, for studying the effects of flavour-changing Yukawa couplings we include these coefficients for completeness. $C_{8}^{g}$ is the WC related to the chromomagnetic operator coming from gluon penguins and the NP contributions $\Delta C_{7,8}^{\prime}$ are computed in [70].

\subsubsection{Penguins and boxes computation}

We review the computation of the WCs in eqs. (3.7)-(3.9) which have been obtained already for both the flavour conserving general THDM in Superlso and for the GTHDM itself in $[15,70,132]$. In these latter works, the Yukawa couplings related to $\xi^{d}$ were 
assumed to be zero or negligibly small from the beginning, avoiding the appearance of possible mixed terms between the down and up couplings that, at first, might not be as suppressed as those involving only down quarks. This computation is performed assuming $\ell=\mu$ in the final state, as inspired by our choice of Yukawa textures in eq. (2.27), but it can be easily generalised for all flavours when required.

Using the model files provided by FeynRules from [133], we generate in FeynArts the one loop level Feynman diagrams for $b \rightarrow s \mu^{+} \mu^{-}$transitions. After this, the amplitudes are tensor decomposed in FeynCalc [134] and then, the resulting Passarino-Veltman functions are Taylor expanded in the external momenta up to second order. Finally, the functions are integrated with Package X [135]. In this way, with $x_{t H^{ \pm}}=m_{t}^{2}\left(\mu_{W}\right) / m_{H^{ \pm}}^{2}$ for $\mu_{W}=$ $\mathcal{O}\left(m_{W}\right)$ we obtain

$$
\begin{aligned}
C_{9}^{\gamma}= & \frac{-\Gamma_{t b}^{L} \Gamma_{t s}^{L}}{\sqrt{2} G_{F} V_{t b} V_{t s}^{*} m_{t}^{2} \lambda_{t t}^{2}} \mathcal{D}^{H(0)}\left(x_{t H^{ \pm}}\right) \\
C_{9}^{Z}= & \frac{\Gamma_{t b}^{L} \Gamma_{t s}^{L}}{\sqrt{2} G_{F} V_{t b} V_{t s}^{*} m_{t}^{2} \lambda_{t t}^{2}} \frac{\left(1-4 s_{W}^{2}\right)}{s_{W}^{2}} \mathcal{C}^{H(0)}\left(x_{t H^{ \pm}}\right)+\frac{m_{b}}{m_{t}} \frac{\Gamma_{t b}^{R} \Gamma_{t s}^{L}}{\sqrt{2} G_{F} V_{t b} V_{t s}^{*}} \mathcal{C}_{\text {mix }}^{H(0)}\left(x_{t H^{ \pm}}\right), \\
C_{9}^{\text {box }}= & C_{10}^{\text {box }}=\frac{\Gamma_{t b}^{L} \Gamma_{t s}^{L}}{32 G_{F}^{2} V_{t b} V_{t s}^{*} m_{t}^{2}}\left|\Gamma_{\nu_{i} \mu}^{R}\right|^{2} \mathcal{B}^{H(0)}\left(x_{t H^{ \pm}}\right) \\
& \quad+\frac{m_{\mu} \xi_{\mu \mu}^{l}}{8 \sqrt{2} G_{F} m_{W}^{3} s_{W}^{2} V_{t b} V_{t s}^{*}} \mathcal{B}_{\text {mix }}^{H(0)}\left(x_{t H^{ \pm}}, H\right), \\
C_{10}^{Z}= & \frac{1}{\left(4 s_{W}^{2}-1\right)} C_{9}^{Z}, \\
C_{7,8}^{\gamma, g}= & \frac{\Gamma_{t b}^{L} \Gamma_{t s}^{L}}{3 \sqrt{2} G_{F} V_{t b} V_{t s}^{*} m_{t}^{2} \lambda_{t t}^{2}} F_{7,8}^{(1)}\left(x_{t H^{ \pm}}\right)-\frac{\Gamma_{t b}^{R} \Gamma_{t s}^{L}}{\sqrt{2} G_{F} V_{t b} V_{t s}^{*} m_{b} m_{t} \lambda_{t t} \lambda_{b b}} F_{7,8}^{(2)}\left(x_{t H^{ \pm}}\right),
\end{aligned}
$$

where

$$
\begin{aligned}
\Gamma_{t s}^{L} & =\frac{1}{\sqrt{2}} \sum_{l=1}^{3} \xi_{l 3}^{u} V_{l 2}^{*}, & \Gamma_{t b}^{L} & =\frac{1}{\sqrt{2}} \sum_{k=1}^{3} V_{k t} \xi_{k 3}^{u *}, \\
\Gamma_{t b}^{R} & =\frac{1}{\sqrt{2}} \sum_{k=1}^{3} V_{k t} \xi_{k 3}^{d *}, & \left|\Gamma_{\nu_{i} \mu}^{R}\right|^{2} & =\frac{1}{2}\left(\left|\xi_{\mu \mu}^{l}\right|^{2}+\left|\xi_{\tau \mu}^{l}\right|^{2}\right),
\end{aligned}
$$

with the Green functions $\mathcal{D}^{H(0)}, \mathcal{C}^{H(0)}, F_{7,8}^{(1)}$ and $F_{7,8}^{(2)}$ defined in appendices $\mathrm{C} 1$ and $\mathrm{C} 2$ in [136]. Here, $\lambda_{i i}$ are the diagonal Yukawa couplings defined in Superlso, $G_{F}$ is the Fermi constant and $s_{W}$ is the sine of the Weinberg angle. The Green function $\mathcal{B}^{H(0)}$ for the box diagram contribution in $C_{9,10}^{\text {box }}$ coming from the new lepton flavour violating (LFV) couplings is given by

$$
\mathcal{B}^{H(0)}(t)=\frac{t(t-t \log t-1)}{m_{W}^{2} s_{W}^{2}(t-1)^{2}} .
$$

Our computation shows two new terms absent in both the Superlso manual and in [70], namely the mixed term in the $C_{9}^{Z}$ expression where

$$
\mathcal{C}_{\text {mix }}^{H(0)}(t)=-\frac{\left(1-4 s_{W}^{2}\right) t\left(t^{2}-2 t \log t-1\right)}{16 m_{W}^{2} s_{W}^{2}(t-1)^{3}},
$$


and a gauge dependent contribution to $C_{9}^{\text {box }}$ coming from the box diagrams in figures $2 \mathrm{c}$ and 2d proportional to $\mathcal{B}_{\text {mix }}^{H(0)}(t, H)$ with $H=m_{H^{ \pm}}^{2} / m_{W}^{2}$ (see appendix A).

For all remaining terms, we obtained full agreement with [70] once the overall $\sqrt{2}$ factor in their Yukawa Lagrangian is taken into account compared to our eq. (2.22). It is important to mention here that once the full quantum field theory matches with the effective theory at a scale $\mu_{W}=\mathcal{O}\left(m_{W}\right)$, the evolution of the WC $C_{7}$ (and $C_{7}^{\prime}$ ) from $\mu=\mu_{W}$ down to $\mu=\mu_{b}$, where $\mu_{b}$ is of the order of $m_{b}$, is given at LO by [137]

$$
C_{7}^{\mathrm{eff}}\left(\mu_{b}\right)=\eta^{\frac{16}{23}} C_{7}+\frac{8}{3}\left(\eta^{\frac{14}{23}}-\eta^{\frac{16}{23}}\right) C_{8}+\sum_{i=1}^{8} h_{i} \eta^{a_{i}} C_{2},
$$

where $\eta=\alpha_{S}\left(\mu_{W}\right) / \alpha_{S}\left(\mu_{b}\right)$ and the renormalisation group evolution of the QCD coupling is

$$
\alpha_{S}\left(\mu_{b}\right)=\frac{\alpha_{S}\left(m_{Z}\right)}{1-\beta_{0} \frac{\alpha_{S}\left(m_{Z}\right)}{2 \pi} \log \left(m_{Z} / \mu_{b}\right)},
$$

with $\beta_{0}=23 / 3$. The $\sum_{i=1}^{8} h_{i} \eta^{a_{i}}$ factor in eq. (3.19) is given in eq. (12.23) of [137] and references therein. The $C_{2}$ coefficient comes from four-quark operators generated by $W$ boson exchange in the SM and contributes importantly when computing the branching ratio $\mathrm{BR}\left(\bar{B} \rightarrow X_{s} \gamma\right)$. In the GTHDM, as shown in [70], an analogous contribution comes from charged Higgs exchange at tree level. In this way, following [138] with $\alpha_{S}\left(m_{Z}\right)=0.117$, we use the following parametric expression at LO:

$$
C_{7}^{\mathrm{eff}}\left(\mu_{b}\right)=0.698 C_{7}+0.086 C_{8}-0.158 C_{2},
$$

where $C_{2}=C_{2}^{\mathrm{SM}}+\Delta C_{2}$ for $C_{2}^{\mathrm{SM}}=1$ and

$$
\Delta C_{2}=-\frac{7}{18} \frac{m_{W}^{2}}{m_{H^{ \pm}}^{2}} \frac{V_{k 2}^{*} \xi_{k 2}^{u} \xi_{n 2}^{u *} V_{n 3}}{g_{2}^{2} V_{t b} V_{t s}^{*}}-\frac{1}{3} \frac{m_{c}}{m_{b}} \frac{m_{W}^{2}}{m_{H^{ \pm}}^{2}} \frac{V_{k 2}^{*} \xi_{k 2}^{u} V_{2 n} \xi_{n 3}^{d}}{g_{2}^{2} V_{t b} V_{t s}^{*}}\left(3+4 \log \left(\frac{\mu_{b}^{2}}{m_{H^{+}}^{2}}\right)\right) .
$$

with $g_{2}$ the weak coupling constant. Similarly, there will be a contribution to the $C_{9}$ (and $C_{9}^{\prime}$ ) WC coming from those four-quark operators given by [70]

$$
C_{9}^{4-\text { quark }}\left(\mu_{b}\right)=\frac{2}{27} \frac{V_{k 2}^{*} \xi_{k 2}^{u} \xi_{n 2}^{u *} V_{n 3}}{g_{2}^{2} V_{t b} V_{t s}^{*}} \frac{m_{W}^{2}}{m_{H^{ \pm}}^{2}}\left(19+12 \log \left(\frac{\mu_{b}^{2}}{m_{H^{ \pm}}^{2}}\right)\right),
$$

which can be added at LO to both the penguins and boxes contributions, obtaining

$$
C_{9}^{\text {eff }}\left(\mu_{b}\right)=C_{9}+C_{9}^{4-\text { quark }}\left(\mu_{b}\right) .
$$

\subsubsection{Summary of contributions}

As already mentioned, in view of the flavour changing couplings in the GTHDM, there are two new contributions compared to the ones present in Superlso. These contributions come from the box diagrams in figures $2 \mathrm{a}-2 \mathrm{~b}$ and from the $Z$ penguin in figure 1 . The $\Gamma_{t b}^{L} \Gamma_{t s}^{L}$ contribution is the largest and dominates the amplitude for most of the parameter space, with a strong dependence on $\tan \beta, m_{H^{ \pm}}, Y_{2, c t / t c}^{u}, Y_{2, t t}^{u}, Y_{2, \mu \mu}^{l}, Y_{2, \mu \tau}^{l}$. There are also 

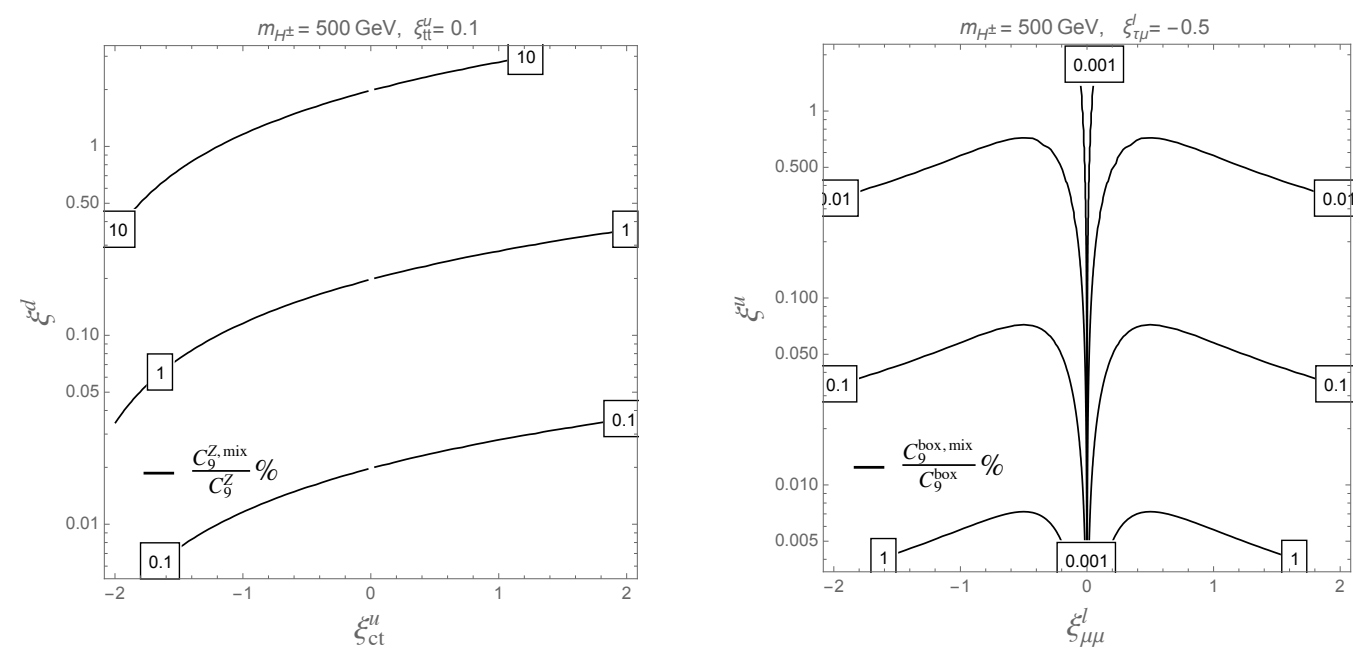

Figure 3. Left: $C_{9}^{Z, \text { mix }} / C_{9}^{Z}$ contour levels for $\xi_{s b}^{d}=\xi_{b b}^{d}=\xi^{d}$. Here $C_{9}^{Z}$ and $C_{9}^{Z, \text { mix }}$ refers to the first and second terms in eq. (3.11) respectively. Right: $C_{9}^{\text {box }, \text { mix }} / C_{9}^{\text {box }}$ contour levels for $\xi_{t t}^{u}=\xi_{c t}^{u}=\xi^{u}$. $C_{9}^{\text {box }}$ and $C_{9}^{\text {box,mix }}$ refers to the first and second terms in eq. (3.12) respectively.

two subdominant contributions, the first one coming from the part proportional to $\Gamma_{t b}^{R} \Gamma_{t s}^{L}$ in the $Z$ penguin diagram in figure 1. When comparing its contribution relative to the $\Gamma_{t b}^{L} \Gamma_{t s}^{L}$ term, we find regions of the parameter space in which it can make up to $10 \%$ of the total contribution (see figure 3 left). The second subdominant contribution is the already mentioned gauge dependent part of the boxes diagrams (figures $2 \mathrm{c}-2 \mathrm{~d}$ ) which is suppressed by the muon mass (see figure 3 right). Additionally we verified that when varying the mass of the charged Higgs from $500 \mathrm{GeV}$ to $4000 \mathrm{GeV}$ these ratios were essentially unaffected. In this way, we keep in our calculations the $\Gamma_{t b}^{R} \Gamma_{t s}^{L}$ term from the $Z$ penguin and neglect the gauge dependent part of the boxes diagrams.

\section{$3.2 \quad b \rightarrow c \ell \bar{\nu}$ semileptonic transitions}

As a consequence of the new interactions between the fermions and the charged Higgs, semileptonic tree level flavour changing transitions appear in the GTHDM (figure 4) which have been extensively studied in the literature [15, 16, 127, 139-141]. Therefore we include tree-level calculations of the Wilson coefficients related to these in our analysis. The effective Hamiltonian responsible for the $b \rightarrow c \ell \bar{\nu}$ transitions for the semileptonic decays of $B$-mesons, including the SM and tree level GTHDM contributions can be written in terms of scalar operators in the form

$$
\mathcal{H}_{\mathrm{eff}}=C_{S M}^{c b} \mathcal{O}_{S M}^{c b}+C_{R}^{c b} \mathcal{O}_{R}^{c b}+C_{L}^{c b} \mathcal{O}_{L}^{c b},
$$

where $C_{S M}^{c b}=4 G_{F} V_{c b} / \sqrt{2}$ and the operators are given by

$$
\begin{aligned}
\mathcal{O}_{S M}^{c b} & =\left(\bar{c} \gamma_{\mu} P_{L} b\right)\left(\bar{\ell} \gamma_{\mu} P_{L} \nu\right) \\
\mathcal{O}_{R}^{c b} & =\left(\bar{c} P_{R} b\right)\left(\bar{\ell} P_{L} \nu\right) \\
\mathcal{O}_{L}^{c b} & =\left(\bar{c} P_{L} b\right)\left(\bar{\ell} P_{L} \nu\right) .
\end{aligned}
$$




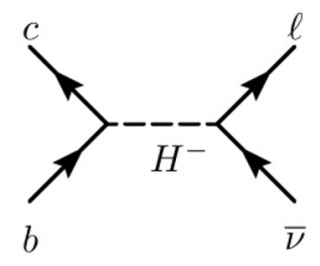

Figure 4. Tree level contribution to $b \rightarrow c \ell \bar{\nu}$.

Given that the flavour of the neutrino in the final state can not be discerned by experiments, one has to add (incoherently) to the SM the NP contributions associated with the LFV couplings $\xi_{i j}^{l}$. As the existing constraints will apply separately to the scalar and the pseudoscalar couplings, it is convenient to define

$$
g_{S}^{\ell \ell^{\prime}} \equiv \frac{C_{R}^{c b}+C_{L}^{c b}}{C_{S M}^{c b}}, g_{P}^{\ell \ell^{\prime}} \equiv \frac{C_{R}^{c b}-C_{L}^{c b}}{C_{S M}^{c b}}
$$

where in our analysis we evaluate the WCs $C_{R}^{c b}$ and $C_{L}^{c b}$ at tree-level, with the expressions,

$$
C_{R}^{c b}=-2 \frac{\left(V_{c b} \xi_{b b}^{d}+V_{c s} \xi_{s b}^{d}\right) \xi_{\ell \ell^{\prime}}^{l *}}{m_{H^{ \pm}}^{2}}, \quad C_{L}^{c b}=2 \frac{V_{t b} \xi_{c c}^{u *} \xi_{\ell \ell^{\prime}}^{l *}}{m_{H^{ \pm}}^{2}} .
$$

\section{Observables}

In this section we present the observables to be included in the fit. We divide them in four sets: the first one for FCNCs in $b \rightarrow s$ transitions and $B$ meson rare decay observables, both of them affected by the new WC contributions. The second set is associated with FCCCs observables that arise from semileptonic $b \rightarrow c \ell \bar{\nu}$ decays and the mass difference $\Delta M_{s}$ from $B_{s}-\bar{B}_{s}$ oscillations. Various leptonic decays of mesons form the third set. Finally, the fourth set contains leptonic observables associated with $\tau$ and $\mu$ decays, among them the anomalous magnetic moment of the muon in particular.

\subsection{FCNCs and $B$ rare decays}

Lepton flavour universality in the SM means that all couplings between leptons and gauge bosons are the same (up to mass differences). This implies that any departure from this identity could be a clear sign of NP. The most interesting tests of LFU violation with FCNC are given by the ratios of $b \rightarrow$ sll transitions

$$
R\left(K^{(*)}\right)=\frac{\Gamma\left(B \rightarrow K^{(*)} \mu^{+} \mu^{-}\right)}{\Gamma\left(B \rightarrow K^{(*)} e^{+} e^{-}\right)}
$$

with $\Gamma$ representing the decay width and $K^{(*)}$ are kaons. As per our choice of Yukawa textures in eq. (2.27), here we only consider NP effects coming from the muon specific WCs, i.e., electronic WCs are SM-like. Aside from this $R\left(K^{(*)}\right)$ ratios, hints for LFU violation are found in many branching fractions and angular observables related to $B \rightarrow$ 
$K^{(*)} \mu^{+} \mu^{-}$decays as a function of the dimuon mass squared $q^{2}$. In this work we use the same observables as in [65], with the predicted values obtained with Superlso and with likelihoods provided via HEPLike. In particular, among the observables included are the optimised angular observables $P_{i}^{(\prime)}$ which have been constructed in order to minimise the hadronic uncertainties emerging from form factor contributions to the $B^{0} \rightarrow K^{* 0} \mu^{+} \mu^{-}$ decay at leading order [142]. In view of that, experimentally these observables are obtained by fitting $q^{2}$-binned angular distributions and they are defined in the theory as CP-averages integrated in the $q^{2}$ bins:

$$
\begin{aligned}
\left\langle P_{1}\right\rangle_{\text {bin }} & =\frac{1}{2} \frac{\int_{\text {bin }} d q^{2}\left[J_{3}+\bar{J}_{3}\right]}{\int_{\text {bin }} d q^{2}\left[J_{2 s}+\bar{J}_{2 s}\right]}, & \left\langle P_{2}\right\rangle_{\text {bin }}=\frac{1}{8} \frac{\int_{\text {bin }} d q^{2}\left[J_{6 s}+\bar{J}_{6 s}\right]}{\int_{\text {bin }} d q^{2}\left[J_{2 s}+\bar{J}_{2 s}\right]}, \\
\left\langle P_{5}^{\prime}\right\rangle_{\text {bin }} & =\frac{1}{2 \mathcal{N}_{\text {bin }}^{\prime}} \int_{\text {bin }} d q^{2}\left[J_{5}+\bar{J}_{5}\right], &
\end{aligned}
$$

where the $J_{i}$ functions and the normalisation constant $\mathcal{N}_{\text {bin }}^{\prime}$ are given in [65]. Additionally, they can be related to the form factor dependent observables $S_{i}[143]$ as

$$
\begin{aligned}
P_{1} & =\frac{2 S_{3}}{\left(1-F_{\mathrm{L}}\right)}, & P_{2}=\frac{2}{3} \frac{A_{\mathrm{FB}}}{\left(1-F_{\mathrm{L}}\right)}, \\
P_{5}^{\prime} & =\frac{S_{5}}{\sqrt{F_{\mathrm{L}}\left(1-F_{\mathrm{L}}\right)}}, &
\end{aligned}
$$

where $A_{\mathrm{FB}}$ is the forward-backward asymmetry of the dimuon system and $F_{L}$ is the fraction of longitudinal polarisation of the $K^{* 0}$ meson.

The most sensitive observable to scalar operators is the branching ratio $\mathrm{BR}\left(B_{s} \rightarrow\right.$ $\mu^{+} \mu^{-}$) which also depends on the muon specific $C_{10}$ and $C_{10}^{\prime}$ WCs [65]:

$$
\begin{aligned}
& \operatorname{BR}( B_{s} \\
&\left.\rightarrow \mu^{+} \mu^{-}\right)=\frac{G_{F}^{2} \alpha^{2}}{64 \pi^{3}} f_{B_{s}}^{2} \tau_{B_{s}} m_{B_{s}}^{3}\left|V_{t b} V_{t s}^{*}\right|^{2} \sqrt{1-\frac{4 m_{\mu}^{2}}{m_{B_{s}}^{2}}} \\
& \times\left[\left(1-\frac{4 m_{\mu}^{2}}{m_{B_{s}}^{2}}\right)\left|\frac{m_{B_{s}}\left(C_{Q_{1}}-C_{Q_{1}}^{\prime}\right)}{\left(m_{b}+m_{s}\right)}\right|^{2}+\left|\frac{m_{B_{s}}\left(C_{Q_{2}}-C_{Q_{2}}^{\prime}\right)}{\left(m_{b}+m_{s}\right)}-2\left(C_{10}-C_{10}^{\prime}\right) \frac{m_{\mu}}{m_{B_{s}}}\right|^{2}\right],
\end{aligned}
$$

where $f_{B_{s}}$ is the decay constant and $\tau_{B_{s}}$ is the mean lifetime.

With respect to the inclusive $\bar{B} \rightarrow X_{s} \gamma$ decay, we use the full expression given in the works of [144-149] and implemented in Superlso. The WCs $C_{7}$ and $C_{7}^{\prime}$ are constrained by this decay, given at the quark level by $b \rightarrow s \gamma$, which at LO is

$$
\Gamma(b \rightarrow s \gamma)=\frac{G_{F}^{2}}{32 \pi^{4}}\left|V_{t b} V_{t s}^{*}\right|^{2} \alpha_{\mathrm{em}} m_{b}^{5}\left(\left|C_{7 \mathrm{eff}}\left(\mu_{b}\right)\right|^{2}+\left|C_{7 \mathrm{eff}}^{\prime}\left(\mu_{b}\right)\right|^{2}\right) .
$$

We also take into account the rare decays $B_{s} \rightarrow \tau^{+} \tau^{-}$and $B^{+} \rightarrow K^{+} \tau^{+} \tau^{-}$as well as the LFV processes $B_{s} \rightarrow \mu^{ \pm} \tau^{\mp}, B^{+} \rightarrow K^{+} \mu^{ \pm} \tau^{\mp}$ and $b \rightarrow s \nu \bar{\nu}$ with theoretical expressions given in [70]. A list of the included FCNC observables ${ }^{3}$ can be found in table 1.

\footnotetext{
${ }^{3}$ New measurements of $\operatorname{BR}\left(B_{s} \rightarrow \mu+\mu^{-}\right)$have been performed recently by LHCb [150, 151], as well as a combination with previous results [64], giving a combined measured value of $2.85_{-0.31}^{+0.34}$. Nevertheless, we do not expect significant deviations from our results with this new measurement.
} 


\begin{tabular}{|c|c|}
\hline Observable & Experiment \\
\hline$R\left(K^{*}\right)[0.045,1.1] \mathrm{GeV}^{2}$ & $0.66 \pm 0.09 \pm 0.03[152]$ \\
\hline$R\left(K^{*}\right)[1.1,6.0] \mathrm{GeV}^{2}$ & $0.69 \pm 0.09 \pm 0.05[152]$ \\
\hline$R(K)[1.1,6.0] \mathrm{GeV}^{2}$ & $0.846 \pm 0.042 \pm 0.013[150]$ \\
\hline $\mathrm{BR}\left(B_{s} \rightarrow \mu^{+} \mu^{-}\right) \times 10^{9}$ & $2.69_{-0.35}^{+0.37}[153]$ \\
\hline $\mathrm{BR}\left(B \rightarrow X_{s} \gamma\right) \times 10^{4}$ & $3.32 \pm 0.15[62]$ \\
\hline $\mathrm{BR}\left(B_{s} \rightarrow \tau^{+} \tau^{-}\right) \times 10^{3}$ & $<6.8$ at $95 \%$ C.L. $[154]$ \\
\hline $\mathrm{BR}\left(B^{+} \rightarrow K^{+} \tau^{+} \tau^{-}\right) \times 10^{3}$ & $<2.25$ at $90 \%$ C.L. $[154]$ \\
\hline $\mathrm{BR}\left(B_{s} \rightarrow \mu^{ \pm} \tau^{\mp}\right) \times 10^{5}$ & $<4.2$ at $95 \%$ C.L. $[154]$ \\
\hline $\mathrm{BR}\left(B^{+} \rightarrow K^{+} \mu^{ \pm} \tau^{\mp}\right) \times 10^{5}$ & $<4.8$ at $90 \%$ C.L. $[154]$ \\
\hline $\mathcal{R}_{K}^{\nu \bar{\nu}}$ & $<3.9$ at $90 \%$ C.L. $[155]$ \\
\hline $\mathcal{R}_{K^{*}}^{\nu \bar{\nu}}$ & $<2.7$ at $90 \%$ C.L. $[155]$ \\
\hline
\end{tabular}

Table 1. Experimental measurements of FCNCs observables and bounds for rare $B$ decays considered in our study. The $\mathcal{R}_{K^{(*)}}^{\nu \bar{\nu}}$ parameters are related to $b \rightarrow s \nu \bar{\nu}$ transitions as introduced in eq. (4.6) in [70]. We also include all the angular distributions and branching fractions of $B^{0} \rightarrow K^{* 0} \mu^{+} \mu^{-}$ decays, the branching fractions of both $B_{s} \rightarrow \phi \mu^{+} \mu^{-}$and $B^{+} \rightarrow K^{+} \mu^{+} \mu^{-}$with measurements provided by the HEPLikeData repository [156].

\subsection{FCCCs observables}

The most relevant FCCC observables are the ratios of semileptonic $B$ meson decays to $\tau$ and light leptons, that is

$$
R\left(D^{(*)}\right)=\frac{\Gamma\left(\bar{B} \rightarrow D^{(*)} \tau \bar{\nu}\right)}{\Gamma\left(\bar{B} \rightarrow D^{(*)} l \bar{\nu}\right)}
$$

where $D^{(*)}$ are charmed mesons and $l$ is either an electron $(e)$ or a muon $(\mu)$. As of the time of writing, the world average for the experimental measurement of the ratios $R\left(D^{(*)}\right)$ sits at a $3.1 \sigma$ deviation from the SM prediction [62].

The GTHDM contributions to $R(D)$ and $R\left(D^{*}\right)$ from the effective Hamiltonian in eq. (3.25) can be written as,

$$
\begin{aligned}
R(D) & =\frac{1+1.5 \operatorname{Re}\left(g_{S}^{\tau \tau}\right)+1.0 \sum\left|g_{S}^{\tau l}\right|^{2}}{3.34+4.8 \sum\left|g_{S}^{\mu l}\right|^{2}}, \\
R\left(D^{*}\right) & =\frac{1+0.12 \operatorname{Re}\left(g_{P}^{\tau \tau}\right)+0.05 \sum\left|g_{P}^{\tau l}\right|^{2}}{3.89+0.25 \sum\left|g_{P}^{\mu l}\right|^{2}} .
\end{aligned}
$$

In addition to $R(D)$ and $R\left(D^{*}\right)$, a third ratio has been measured by the Belle collaboration [157], the ratio $R_{e / \mu}=\operatorname{BR}(\bar{B} \rightarrow D e \bar{\nu}) / \mathrm{BR}(\bar{B} \rightarrow D \mu \bar{\nu})$ which is considered to be 
the stringent test of LFU in $B$ decays. It can be expressed in the GTHDM as

$$
R_{e / \mu}=\frac{1}{0.9964+0.18 \operatorname{Re}\left(g_{S}^{\mu \mu}\right)+1.46 \sum\left|g_{S}^{\mu l}\right|^{2}},
$$

where we have obtained the NP leptonic contributions by integrating the heavy quark effective theory (HQET) amplitudes of the scalar type operators from [158, 159].

The $B_{c}$ meson lifetime has contributions from the SM, given by $\tau_{B_{c}}^{\mathrm{SM}}=0.52_{-0.12}^{+0.18}$ ps [160], and the GTHDM, which can be written as

$$
\begin{aligned}
1 / \tau_{B_{c}}^{\mathrm{GTHDM}}=\Gamma_{B_{c} \rightarrow \tau \bar{\nu}}^{\mathrm{GTHDM}}= & \frac{m_{B_{c}}\left(m_{\tau} f_{B_{c}} G_{F}\right)^{2}\left|V_{c b}\right|^{2}}{8 \pi}\left(1-\frac{m_{\tau}^{2}}{m_{B_{c}}^{2}}\right)^{2} \\
& \times\left[\left|1+\frac{m_{B_{c}}^{2}}{m_{\tau}\left(m_{b}+m_{c}\right)} g_{P}^{\tau \tau}\right|^{2}+\left|\frac{m_{B_{c}}^{2}}{m_{\tau}\left(m_{b}+m_{c}\right)} g_{P}^{\tau l}\right|^{2}-1\right],
\end{aligned}
$$

where the -1 term accounts for the subtraction of the SM contribution. By using the lifetime of the $B_{c}$ meson as the constraining observable, we can compare it to the current experimental measurement of $\tau_{B_{c}}=0.510 \pm 0.009(\mathrm{ps})$ [154], instead of using the theoretical limits on the branching ratio $\operatorname{BR}\left(B_{c} \rightarrow \tau \bar{\nu}\right)$, which are reported to be either $10 \%$ [161] and $30 \%$ [141]. ${ }^{4}$

Another related measurement, $B_{c}^{+} \rightarrow J / \psi \tau^{+} \nu_{\tau}$, has been reported by LHCb [165] and also hints to disagreement with the SM. However the errors are too large at present to reach a definitive conclusion, with $\mathcal{R}(J / \psi)=0.71 \pm 0.17 \pm 0.18$. In addition it has been claimed that the hadronic uncertainties are not at the same level as for the observables related to $\bar{B} \rightarrow D^{*}$ transitions [158], so we do not include it in our fit.

In contrast, a recent measurement of the longitudinal polarization fraction of the $D^{*}$ meson, defined as

$$
F_{L}\left(D^{*}\right)=\frac{\Gamma_{\lambda_{D^{*}}=0}\left(\bar{B} \rightarrow D^{*} \tau \bar{\nu}\right)}{\Gamma\left(\bar{B} \rightarrow D^{*} \tau \bar{\nu}\right)},
$$

has been recently announced by the Belle collaboration [166],

$$
F_{L}\left(D^{*}\right)=0.6 \pm 0.08 \text { (stat) } \pm 0.04 \text { (syst) }
$$

deviating from the SM prediction $F_{L}^{\mathrm{SM}}\left(D^{*}\right)=0.457 \pm 0.010$ [167] by $1.6 \sigma$. The $B \rightarrow D^{*} \tau \bar{\nu}$ differential decay width into longitudinally-polarized $\left(\lambda_{D^{*}}=0\right) D^{*}$ mesons is given (keeping NP from scalar contributions only) by

$$
\begin{array}{r}
\frac{d \Gamma_{\lambda_{D^{*}=0}}^{D^{*}}=\frac{G_{F}^{2}\left|V_{c b}\right|^{2}}{d q^{2}}}{192 \pi^{3} m_{B}^{3}} q^{2} \sqrt{\lambda_{D^{*}}\left(q^{2}\right)}\left(1-\frac{m_{\tau}^{2}}{q^{2}}\right)^{2}\left\{\left[\left(1+\frac{m_{\tau}^{2}}{2 q^{2}}\right) H_{V, 0}^{2}+\frac{3}{2} \frac{m_{\tau}^{2}}{q^{2}} H_{V, t}^{2}\right]\right. \\
\left.+\frac{3}{2}\left|C_{R}^{c b}-C_{L}^{c b}\right|^{2} H_{S}^{2}+3 \operatorname{Re}\left(C_{R}^{c b *}-C_{L}^{c b *}\right) \frac{m_{\tau}}{\sqrt{q^{2}}} H_{S} H_{V, t}\right\},
\end{array}
$$

\footnotetext{
${ }^{4}$ In $[162]$ it was found that values even as large as $60 \%$ could not be excluded, in agreement with a recent calculation of the SM prediction [163, 164].
} 


\begin{tabular}{|c|c|}
\hline Observable & Experiment \\
\hline$R(D)$ & $0.340 \pm 0.027 \pm 0.013[62]$ \\
\hline$R\left(D^{*}\right)$ & $0.295 \pm 0.011 \pm 0.008[62]$ \\
\hline$R_{e / \mu}$ & $1.01 \pm 0.01 \pm 0.03[157]$ \\
\hline$\tau_{B_{c}}(\mathrm{ps})$ & $0.510 \pm 0.009[154]$ \\
\hline$F_{L}\left(D^{*}\right)$ & $0.6 \pm 0.08 \pm 0.04[166]$ \\
\hline$\Delta M_{s}\left(\mathrm{ps}^{-1}\right)$ & $17.741 \pm 0.020[62]$ \\
\hline
\end{tabular}

Table 2. Observables related to the charged anomalies considered in our study. We also include the normalised distributions $d \Gamma(B \rightarrow D \tau \bar{\nu}) /\left(\Gamma d q^{2}\right)$ and $d \Gamma\left(B \rightarrow D^{\star} \tau \bar{\nu}\right) /\left(\Gamma d q^{2}\right)$ as measured by the BaBar collaboration [168].

where the helicity amplitudes are defined in appendix B of [158]. In addition, we also include the normalised distributions $d \Gamma(B \rightarrow D \tau \bar{\nu}) /\left(\Gamma d q^{2}\right)$ and $d \Gamma\left(B \rightarrow D^{\star} \tau \bar{\nu}\right) /\left(\Gamma d q^{2}\right)$, as measured by the BaBar collaboration [168].

Lastly, the mass difference $\Delta M_{s}$ of $B_{s}-\bar{B}_{s}$ oscillations is included in our study and (for $m_{A}=m_{H}$ ) is given by [71]

$$
\begin{aligned}
\Delta M_{s}^{\mathrm{GTHDM}}= & -\frac{f_{B_{s}}^{2} M_{B_{s}}^{3}}{4\left(m_{b}+m_{s}\right)^{2}}\left[c_{\beta \alpha}^{2}\left(\frac{1}{m_{h}^{2}}-\frac{1}{m_{H}^{2}}\right)+\frac{2}{m_{H}^{2}}\right] \\
& \times\left\{\left(U_{22} \tilde{\mathcal{B}}_{B_{s}}^{(2)} b_{2}+U_{32} \tilde{\mathcal{B}}_{B_{s}}^{(3)} b_{3}\right)\left[\left(\xi_{b s}^{d *}\right)^{2}+\left(\xi_{s b}^{d}\right)^{2}\right]+2\left(U_{44} \tilde{\mathcal{B}}_{B_{s}}^{(4)} b_{4}\right) \xi_{b s}^{d *} \xi_{s b}^{d}\right\},
\end{aligned}
$$

with $\vec{b}=\{8 / 3,-5 / 3,1 / 3,2,2 / 3\}$, bag factors $\tilde{\mathcal{B}}_{B_{s}}^{(2)}=0.806, \tilde{\mathcal{B}}_{B_{s}}^{(3)}=1.1$ and $\tilde{\mathcal{B}}_{B_{s}}^{(4)}=$ $1.022[169,170]$, and the $U$ running matrix being defined in [71]. A summary of all FCCC observables included in this study is provided in table 2 .

\subsection{Leptonic decays of mesons}

Beyond those described in sections 4.1 and 4.2, there are additional leptonic decays included in this study. The total decay width at LO for the process $M \rightarrow l \nu$ in the GTHDM is computed as $[126,132,171]$

$$
\operatorname{BR}\left(M_{i j} \rightarrow l \nu\right)=G_{F}^{2} m_{l}^{2} f_{M}^{2} \tau_{M}\left|V_{i j}\right|^{2} \frac{m_{M}}{8 \pi}\left(1-\frac{m_{l}^{2}}{m_{M}^{2}}\right)^{2}\left[\left|1-\Delta_{i j}^{l l}\right|^{2}+\left|\Delta_{i j}^{l l^{\prime}}\right|^{2}\right],
$$

where $i, j$ are the valence quarks of the meson $M, f_{M}$ is its decay constant and $\Delta_{i j}^{l l^{\prime}}$ is the NP correction given by

$$
\Delta_{i j}^{l l^{\prime}}=\left(\frac{m_{M}}{m_{H^{ \pm}}}\right)^{2} Z_{l l^{\prime}}\left(\frac{Y_{i j} m_{u_{i}}+X_{i j} m_{d_{j}}}{V_{i j}\left(m_{u_{i}}+m_{d_{j}}\right)}\right), \quad l, l^{\prime}=2,3 .
$$

where the relations

$$
X_{i j}=\frac{v}{\sqrt{2} m_{d_{j}}} V_{i k} \xi_{k j}^{d}, \quad Y_{i j}=-\frac{v}{\sqrt{2} m_{u_{i}}} \xi_{k i}^{u *} V_{k j}, \quad Z_{i j}=\frac{v}{\sqrt{2} m_{j}} \xi_{i j}^{l},
$$

depend on the Yukawa textures. The list of fully leptonic decays of mesons included in this analysis, for various mesons $M$, can be seen in table 3 . 


\begin{tabular}{|c|c|}
\hline Observable & Experiment \\
\hline $\mathrm{BR}\left(B_{u} \rightarrow \tau \nu\right) \times 10^{4}$ & $1.09 \pm 0.24[172]$ \\
\hline$\frac{\mathrm{BR}(K \rightarrow \mu \nu)}{\mathrm{BR}(\pi \rightarrow \mu \nu)}$ & $0.6358 \pm 0.0011[115]$ \\
\hline $\mathrm{BR}\left(D_{s} \rightarrow \tau \nu\right) \times 10^{2}$ & $5.48 \pm 0.23[173]$ \\
\hline $\mathrm{BR}\left(D_{s} \rightarrow \mu \nu\right) \times 10^{3}$ & $5.49 \pm 0.16[173]$ \\
\hline $\mathrm{BR}(D \rightarrow \mu \nu) \times 10^{4}$ & $3.74 \pm 0.17[154]$ \\
\hline $\mathrm{BR}(D \rightarrow \tau \nu) \times 10^{3}$ & $1.20 \pm 0.27[174]$ \\
\hline
\end{tabular}

Table 3. Additional leptonic decays of mesons considered in this work.

\subsection{Leptonic observables}

There are a number of leptonic processes that are forbidden or suppressed in the SM but can occur in the GTHDM. These include modifications to the form factors for $\ell \ell^{\prime} \gamma, \ell \ell^{\prime} Z$ and other interactions, which lead to contributions to the anomalous magnetic moment of the muon, $(g-2)_{\mu}$, and LFV decays such as $\tau \rightarrow \mu \gamma, \tau \rightarrow 3 \mu$ and $h \rightarrow \tau \mu$. In the $\mathrm{SM}$, the contributions to these LFV observables are suppressed by the GIM mechanism, giving a very low experimental background, but in the GTHDM LFV is allowed at oneand two-loop level through the couplings $\xi_{i j}^{l}$ in eqs. (2.24)-(2.26), (B.3). ${ }^{5}$

A second Higgs doublet has been examined as a way to explain the muon $g-2$ anomaly. In the Type-X [18-31] and Flavour-Aligned [32-37] versions of the THDM the contributions from two-loop diagrams are dominant in most of the parameter space thanks to mechanisms also available in the GTHDM. Additionally, with LFV, the one-loop diagrams can receive a chirality flip enhancement from including the tau lepton in the diagram loop, as was investigated by [38-41, 43-46], however they only examined muon $g-2$ contributions at the one-loop level.

Due to the similarity of the diagrams between $\ell \rightarrow \ell^{\prime} \gamma$ and muon $g-2$ (which is effectively $\mu \rightarrow \mu \gamma$, see figure 5), these two observables share nomenclature and contributions. For both muon $g-2$ and $\tau \rightarrow \mu \gamma$ we can break the contributions into the same three groups: one-loop, $A_{i j L, R}^{(1)}$; two-loop fermionic, $A_{i j L, R}^{(2, f)}$; and two-loop bosonic, $A_{i j L, R}^{(2, b)}$, contributions, so that the observables can be written as

$$
\begin{aligned}
\Delta a_{\mu}^{\mathrm{GTHDM}} & =m_{\mu}^{2}\left(A_{\mu \mu L}^{(1)}+A_{\mu \mu R}^{(1)}+A_{\mu \mu}^{(2, f)}+A_{\mu \mu}^{(2, b)}\right), \\
\frac{\operatorname{BR}(\tau \rightarrow \mu \gamma)}{\operatorname{BR}\left(\tau \rightarrow \mu \bar{\nu}_{\mu} \nu_{\tau}\right)} & =\frac{48 \pi^{3} \alpha_{\mathrm{EM}}\left(\left|A_{\tau \mu L}\right|^{2}+\left|A_{\tau \mu R}\right|^{2}\right)}{G_{F}^{2}},
\end{aligned}
$$

with $A_{\tau \mu L, R}=A_{\tau \mu L, R}^{(1)}+A_{\tau \mu L, R}^{(2, f)}+A_{\tau \mu L, R}^{(2, b)}$ and $\alpha_{E M}$ is the fine structure constant. All form factors $A_{i j L, R}^{(l)}$ have been appropriately renormalised by combining with the relevant counterterms, and are all calculated using masses and couplings that have been extracted from data at tree-level. Additionally, for the contributions to muon $g-2$ we must subtract

\footnotetext{
${ }^{5}$ Note that in this study we will focus solely on the decays involving $\tau$ and $\mu$ leptons due to our choice of including only second and third generations in the $\xi_{i j}^{l}$ matrix from eq. (2.27).
} 

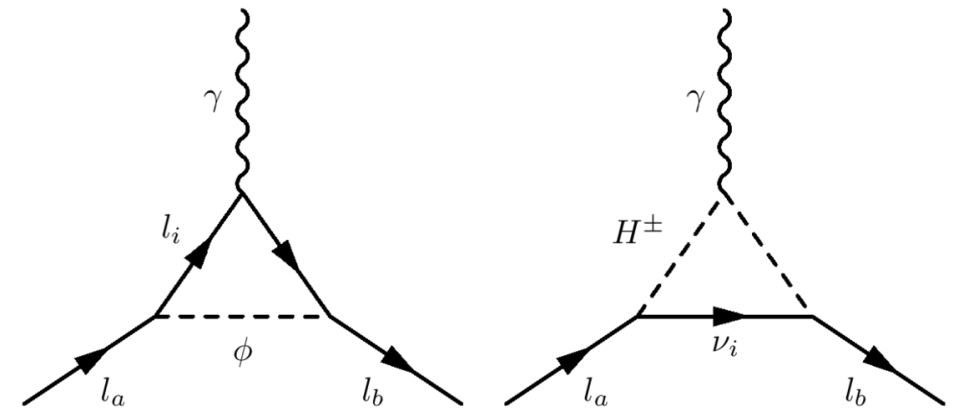

Figure 5. One-loop diagrams contributing to $\ell \rightarrow \ell^{\prime} \gamma$ with a neutral scalar diagram on the left and a charged scalar diagram on the right. The indices $a, b, i$ correspond to any of the lepton flavours $e, \mu, \tau$, and we have $\phi=h, H, A$.
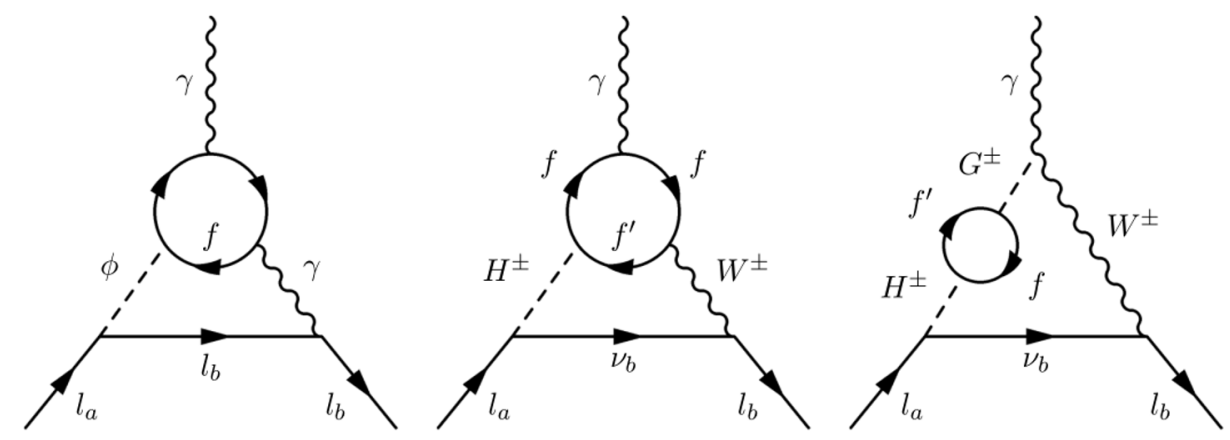

Figure 6. Two-loop fermionic Barr-Zee diagrams contributing to muon $g-2$ and $l \rightarrow l^{\prime} \gamma$. The indices $a, b$ correspond to any of the lepton flavours $e, \mu, \tau$, and $\phi=h, H, A$. The internal photon $\gamma$ may be replaced by a $Z$ boson.

off the SM contributions from the SM Higgs boson to obtain a purely BSM contribution to muon $g-2$.

The entire one loop contribution for muon $g-2$ and $\ell \rightarrow \ell^{\prime} \gamma$ can be found by summing over the neutral scalars $\phi$ and lepton generations:

$$
A_{a b L, R}^{(1)}=\sum_{i=e, \mu, \tau}^{3} \sum_{\phi=h, H, A}\left(A_{a b L, R}^{(F F S)}(\phi, i)-A_{a b L, R}^{(S S F)}\left(H^{ \pm}, i\right)\right),
$$

where the functions $A_{a b L, R}^{(F F S)}(\phi, i)$ and $A_{a b L, R}^{(S S F)}(\phi, i)$ involve neutral scalars $(h, H, A)$ and the charged scalar $H^{ \pm}$respectively. They are defined in eqs. (B.1)-(B.2) in appendix B, and shown in figure 5. To obtain the BSM contributions to muon $g-2$, we must also subtract off the contribution from the SM Higgs boson to obtain a truly-BSM one-loop contribution. 

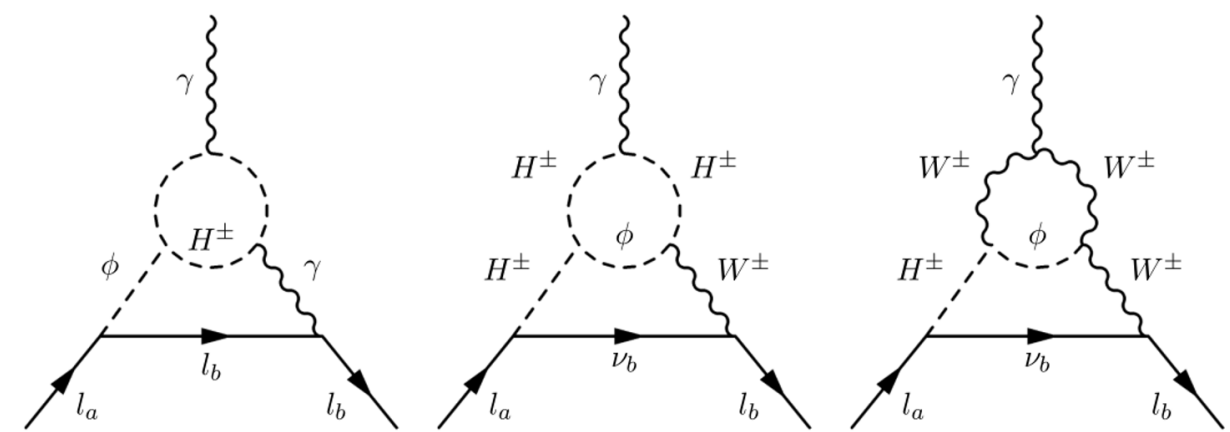

Figure 7. Two-loop bosonic Barr-Zee diagrams contributing to muon $g-2$ and $l \rightarrow l^{\prime} \gamma$. The indices $a, b$ correspond to any of the lepton flavours $e, \mu, \tau$, and we have $\phi=h, H, A$. In the left panel, the internal photon $\gamma$ may be replaced by a $Z$ boson, and the internal $H^{ \pm}$with a $W^{ \pm}$boson.

At the two-loop level we consider the Barr-Zee diagrams, shown in figures 6 and 7 . Just as for the one-loop contributions before, we can subdivide each of these contributions into diagrams involving charged leptons $\left(l_{i}^{-}\right)$paired with neutral bosons $(h, H, A, Z, \gamma)$ and neutral leptons $\left(\nu_{i}\right)$ paired with charged bosons $\left(H^{ \pm}, W^{ \pm}\right) .{ }^{6}$ The two-loop bosonic and fermionic diagrams involve an internal loop made of either bosons or fermions respectively. The total fermionic two-loop contribution to muon $g-2$ is given by [34]

$$
A_{\mu \mu}^{(2, f)}=\sum_{f=u, d, l}\left(A_{\mu \mu}^{(F C)}\left(H^{ \pm}, f\right)+\sum_{\phi=h, H, A} A_{\mu \mu}^{(F N)}(\phi, f)-A_{\mu \mu}^{(F N)}\left(h_{S M}, f\right)\right),
$$

where the form factors are given in eqs. (B.4)-(B.5) in appendix B. Note that only contributions from the heaviest generations of the fermions are considered, via $\Gamma_{\phi 33}^{f}(f=u, d, e)$. Similarly the total bosonic two-loop contributions to muon $g-2$ are

$$
A_{\mu \mu}^{(2, b)}=\sum_{\phi=h, H}\left(A_{\mu \mu}^{(B H N)}(\phi)+A_{\mu \mu}^{(B W N)}(\phi)+A_{\mu \mu}^{(B H C)}(\phi)+A_{\mu \mu}^{(B W C)}(\phi)\right)-A_{\mu \mu}^{(B W N)}\left(h_{S M}\right),
$$

where again the bosonic two-loop functions are in eqs. (B.17)-(B.20) in the same appendix. Note that these contributions do not include 2-loop diagrams with an internal $Z$ boson leg, as in [32].

In the case of the $\tau \rightarrow \mu \gamma$ decay, the contributions from the fermionic and bosonic Barr-Zee two loop diagrams, $A_{a b L, R}^{(2, f)}$ and $A_{a b L, R}^{(2, b)}$ respectively, have the same form for each

\footnotetext{
${ }^{6}$ We do not consider two-loop bosonic diagrams that are not Barr-Zee diagrams, since their maximum contributions to muon $g-2$ are relatively small [35], whereas Barr-Zee contributions have been proved to be dominant for some regions of the parameter space [175]. Additionally, two-loop diagrams involving neutral bosons where both legs are Higgs bosons are suppressed by a factor $m_{\mu}^{4}$, while diagrams with both legs being either $\gamma$ or $Z$ are SM contributions, so we do not consider either, only those with both a $\phi$ and a $\gamma$ or $Z$ boson leg. Similarly for diagrams involving charged legs of $H^{ \pm}, W^{ \pm}$, we only consider a $H^{ \pm}$and $W^{ \pm}$boson paired together, as a pair of $H^{ \pm}$legs lead to diagrams with suppressed contributions [32].
} 
Higgs bosons and fermion or boson in the loop, and can be found in eqs. (B.27), (B.28) in appendix B.

The contributions to $\tau \rightarrow 3 \mu$ decay can be divided up into 3 separate groups, the tree-level, dipole, and the contact contributions. The contributions from tree-level decay are computed in [127]. We have found that the dipole contributions, which involve the penguin-photon diagrams of the form of $\tau \rightarrow \mu \gamma$ decays, are quite sizable compared to those at tree-level and cannot be ignored. Namely, they are given by [176]:

$$
\mathrm{BR}(\tau \rightarrow 3 \mu)^{(\text {dipole })}=\frac{\alpha_{\mathrm{EM}}}{3 \pi}\left(\log \left(\frac{m_{\tau}^{2}}{m_{\mu}^{2}}\right)-\frac{11}{4}\right) \frac{\mathrm{BR}(\tau \rightarrow \mu \gamma)}{\operatorname{BR}\left(\tau \rightarrow \mu \bar{\nu}_{\mu} \nu_{\tau}\right)} .
$$

Similarly, the contact terms involving effective four-fermion interactions [177] could be at first comparable to the dipole contributions. The contact contributions are given by

$$
\operatorname{BR}(\tau \rightarrow 3 \mu)^{(\text {contact })}=\frac{\left|g_{2}\right|^{2}}{8}+2\left|g_{4}\right|^{2}+\frac{16 \pi \alpha_{e m}}{\sqrt{2} G_{F}} \operatorname{Re}\left(g_{4}^{*}\left(A_{\tau \mu L, R}^{(1)}+A_{\tau \mu L, R}^{(2, f)}+A_{\tau \mu L, R}^{(2, b)}\right)\right),
$$

where the coefficients $g_{2}$ and $g_{4}$ are given in appendix B.

Another observable that we include is the lepton violating $h \rightarrow \tau \mu$ decay. This is given at tree level by ${ }^{7}$

$$
\mathrm{BR}(h \rightarrow \tau \mu)=\frac{3 c_{\beta \alpha}^{2} m_{h}}{8 \pi \Gamma_{h}}\left(\left|\xi_{\mu \tau}^{l}\right|^{2}+\left|\xi_{\tau \mu}^{l}\right|^{2}\right)\left(1-\frac{m_{\tau}^{2}}{m_{h}^{2}}\right)^{2}
$$

with the total decay width of $h$ given by $\Gamma_{h}=3.2 \mathrm{MeV}[174]$.

Lastly, besides $g-2$ and LFV observables, experiments have also provided constraints for the LFU ratio in $\tau$ decays. This ratio is commonly known as $\left(g_{\mu} / g_{e}\right)^{2}$ and is given as $[126,171]$

$$
\left(\frac{g_{\mu}}{g_{e}}\right)^{2}=\frac{\operatorname{BR}(\tau \rightarrow \mu \bar{\nu} \nu)}{\operatorname{BR}(\tau \rightarrow e \bar{\nu} \nu)} \frac{f\left(m_{e}^{2} / m_{\tau}^{2}\right)}{f\left(m_{\mu}^{2} / m_{\tau}^{2}\right)} \simeq 1+\sum_{i, j=\mu, \tau}\left(0.25 R_{i j}^{2}-0.11 R_{i i}\right)
$$

where $f(x)=1-8 x+8 x^{3}-x^{4}-12 x^{2} \log x$ and $R_{i j}$ is the BSM scalar contribution, given in the GTHDM as

$$
R_{i j}=\frac{v^{2}}{2 m_{H^{ \pm}}^{2}}\left(\xi_{\tau i}^{l} \xi_{j \mu}^{l}\right)
$$

All of the experimental measurements and upper bounds for leptonic observables are shown in table 4.

\footnotetext{
${ }^{7}$ We computed the contributions coming from one-loop diagrams with two charged Higgses in the loop and found them to be 7 orders of magnitude suppressed compared to the tree level. Diagrams involving a pair of heavy neutral Higgses are possible as well but even more suppressed. The GTHDM only takes into account the tree level, which relies on being close to the alignment limit but not exactly, otherwise this tree level contribution would be zero.
} 


\begin{tabular}{|c|c|}
\hline Observable & Experiment \\
\hline$\Delta a_{\mu}$ & $2.51 \pm 59 \times 10^{-9}[72]$ \\
\hline $\mathrm{BR}(\tau \rightarrow \mu \gamma)$ & $<4.4 \times 10^{-8}$ at $90 \%$ C.L. $[154]$ \\
\hline $\mathrm{BR}(\tau \rightarrow 3 \mu)$ & $<2.1 \times 10^{-8}$ at $95 \%$ C.L. $[154]$ \\
\hline $\mathrm{BR}(h \rightarrow \tau \mu)$ & $<1.5 \times 10^{-3}$ at $95 \%$ C.L. $[178]$ \\
\hline$\left(g_{\mu} / g_{e}\right)$ & $1.0018 \pm 0.0014[179]$ \\
\hline
\end{tabular}

Table 4. World average measurement of $\Delta a_{\mu}$ and experimental bounds for the LFV decay and LFU observables considered in our analysis.

\section{Results}

Our main goal is to study the impact of these observables on the GTHDM parameter space and, in particular, infer the goodness-of-fit of the model in light of these anomalies. Given the plethora of observables defined in the previous section and the large multidimensional parameter space, it is very important to combine them in a statistically rigorous manner in a global fit. This avoids serious shortcomings from more naive approaches like simply overlaying constraints from confidence intervals [180].

To visualize the results we will project the high dimensional parameter space onto twodimensional planes. To this end, the central quantity of interest is the profile likelihood,

$$
\log \mathcal{L}_{\text {prof }}\left(\theta_{1}, \theta_{2}\right)=\max _{\boldsymbol{\eta}} \log \mathcal{L}\left(\theta_{1}, \theta_{2}, \boldsymbol{\eta}\right)
$$

which is, for fixed parameters of interest $\theta_{1}$ and $\theta_{2}$, the maximum value of the log-likelihood function that can be obtained when maximizing over the remaining parameters $\boldsymbol{\eta}$. All profile likelihood figures in this study are created with pippi [181].

As mentioned earlier, we use here the GAMBIT framework for our study. The theoretical predictions of the model and the experimental likelihoods are either implemented natively in GAMBIT or from external tools interfaced with GAMBIT. In particular, the likelihoods related to $b \rightarrow s \mu^{+} \mu^{-}$transitions are obtained from HEPLike, which retrieves experimental results and their correlated uncertainties from the HEPLikeData repository. To efficiently explore the parameter space, we employ the differential evolution sampler Diver, which is a self-adaptive sampler. We choose a population size of NP $=20000$ and a convergence threshold of convthresh $=10^{-6}$. The data we present in this work comes from scans that took between 6 and 8 hours of running time on the Australian supercomputer GADI with cores varying between 1400 and 2000 .

\subsection{Parameter space}

We perform the parameter scans in the physical basis, i.e., where the tree-level masses of the heavy Higgses, $m_{H}, m_{A}$ and $m_{H^{ \pm}}$are taken as input. The remaining model parameters are $\tan \beta, m_{12}$ and the Yukawa couplings $Y_{2, i j}^{f}$ as in eq. (2.23). In order to avoid collider constraints, we work in the alignment limit choosing $s_{\beta-\alpha}$ close to 1 , and we select a 

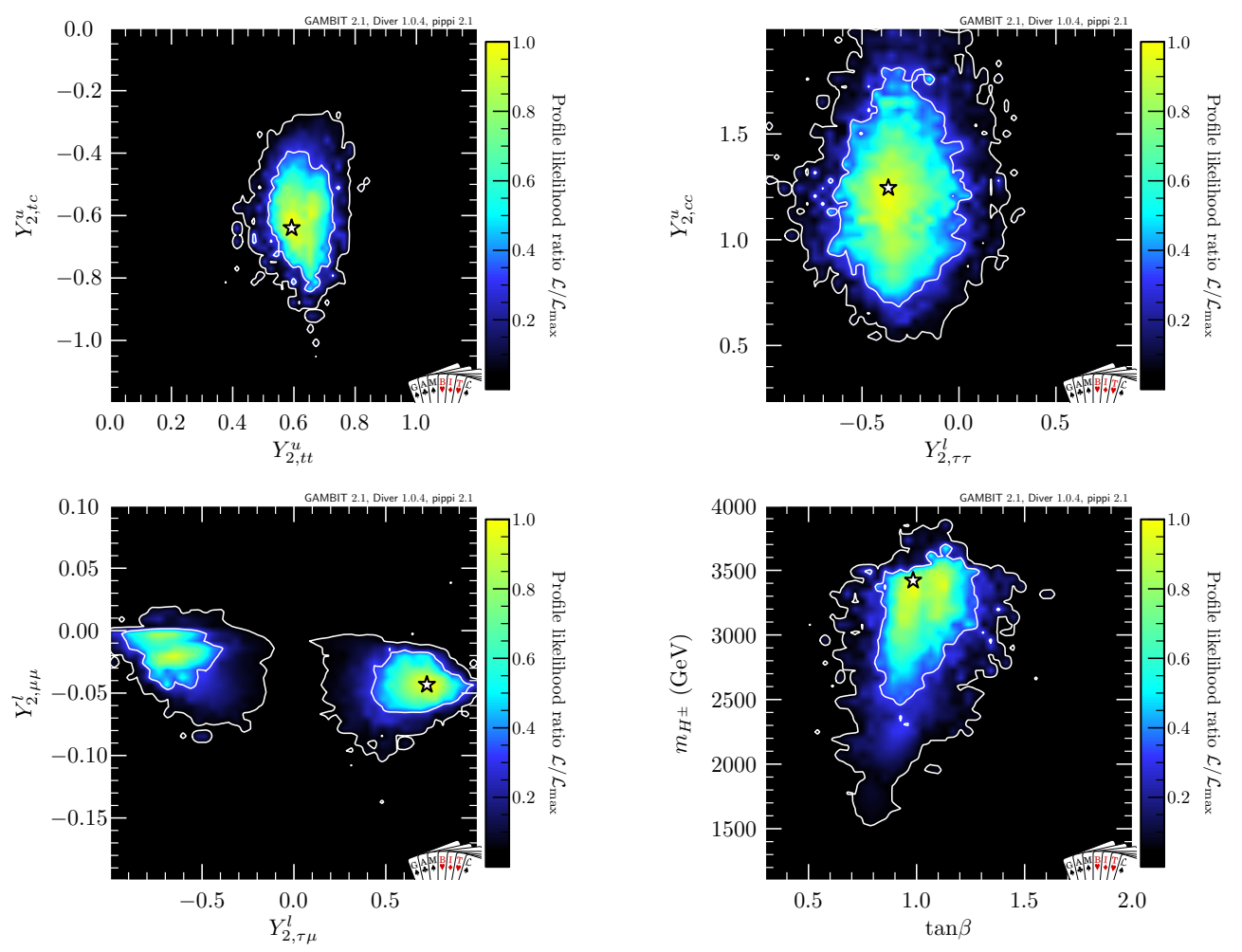

Figure 8. Profile likelihood ratios $\mathcal{L} / \mathcal{L}_{\max }$ for different $2 \mathrm{D}$ plots of the parameter space for $Y_{2, t c}^{u} \in$ $[-2,0]$.

conservative lower limit on the masses of the heavy Higgses $m_{H, A, H^{ \pm}} \geq 500 \mathrm{GeV} .{ }^{8}$ We also fix $m_{A}=m_{H}$ in our study, motivated by the requirement to satisfy the oblique parameter constraints which favour small mass splittings and in order to simplify the sampling of the parameter space. So as to choose reasonable priors for the Yukawa couplings, we take into account various constraints on them (or equivalently on $\xi_{i j}^{f}$ ) from previous studies. The tighter theoretical constraints come from perturbativity which requires $\left|\xi_{i j}^{f}\right| \leq \sqrt{4 \pi} \sim 3.5$. On the phenomenological side, the studies in $[43,131]$ have found values as large as $\xi_{t t}^{u} \sim 0.1$ and $\xi_{t c}^{u} \sim 0.32$ for masses of the heavy Higgses of order $500 \mathrm{GeV}$. With respect to the $\xi_{c c}^{u}$ coupling, it has been shown in [132] that $\mathcal{O}(1)$ values are possible within the charged anomalies, and similar values were considered in [70] in the context of the neutral anomalies, not only for $\xi_{c c}^{u}$ but for all the Yukawa matrix elements. As for the new leptonic couplings, the results in $[15,70,175]$ indicate they should be $\mathcal{O}(1)$ or less in order to fit the charged anomalies. Lastly, the extra down Yukawa couplings $\xi_{i j}^{d}$ are in general expected to be $\mathcal{O}(0.1)[40,183]$ and in particular $\xi_{s b}^{d}$ is expected to be strongly constrained by $B_{s}-\bar{B}_{s}$

\footnotetext{
${ }^{8}$ From preliminary results we found that low Higgs masses are disfavoured by the contribution of various constraints and thus we do not attempt to include precise constraints on the masses from BSM Higgs searches (see e.g. [182] for a discussion of the limits on the charged Higgs mass). We leave a detailed collider study to future work.
} 
mixing. With all these considerations, the chosen priors on our scan parameters are

$$
\begin{aligned}
& \tan \beta \in[0.2,50], \quad m_{12} \in[-1000,2700] \mathrm{GeV}, \quad m_{H^{ \pm}}, m_{A}=m_{H} \in[500,4000] \mathrm{GeV}, \\
& Y_{2, t t}^{u} \in[0.0,2.0], \quad Y_{2, c c}^{u}, Y_{2, t c}^{u} \in[-2.0,2.0], \\
& Y_{2, b b}^{d} \in[-0.10 .1], \quad \quad Y_{2, s s}^{d} \in[-0.2,0.2], \\
& Y_{2, \mu \mu}^{l} \in[-0.5,0.5], \quad Y_{2, \tau \tau}^{l}, Y_{2, \mu \tau}^{l}=Y_{2, \tau \mu}^{l} \in[-1.0,1.0] \text {, } \\
& Y_{2, s b}^{d}=Y_{2, b s}^{d} \in[-0.01,0.01]
\end{aligned}
$$

The results of our scans show two degenerate regions of solutions according to the sign of $Y_{2, t c}^{u}$. We indeed verified that these regions are degenerate and the final results are unaffected by this choice, hence we select $Y_{2, t c}^{u} \in[-2,0]$ for the phenomenological analysis from now on. Namely, this degeneracy is a result of the dependency of various observables on products like $Y_{2, t c}^{u} Y_{2, i j}^{f}$ where $Y_{2, i j}^{f}$ also flips its sign. ${ }^{9}$

We show in figure 8 different 2D planes with the most relevant parameters obtained by the scan. The values for $Y_{2, t t}^{u}$ and $Y_{2, t c}^{u}$ are displayed in the top left panel where we can observe that for the best fit point $\left|Y_{2, t t}^{u}\right| \approx\left|Y_{2, t c}^{u}\right| \approx 0.6$. Then, in the top right panel we see a preferred value for $Y_{2, c c}^{u} \approx 1.1$ (-1.1 for the positive sign solution of $Y_{2, t c}^{u}$ from the degeneracy of solutions). This, along with the lepton Yukawa couplings $Y_{2, \mu \mu}^{l}$ and $Y_{2, \tau \mu}^{l}$ (bottom right panel), helps to enhance the contributions from the box diagrams in figures $2 \mathrm{a}-2 \mathrm{~b}$. Additionally, the LFV coupling $Y_{2, \tau \mu}^{l}$ also contributes to the $B^{+} \rightarrow K^{+} \mu^{ \pm} \tau^{\mp}$ decay, requiring $\left|Y_{2, \tau \mu}^{l}\right| \gtrsim 0.4$ in order to get $\operatorname{BR}\left(B^{+} \rightarrow K^{+} \mu^{ \pm} \tau^{\mp}\right) \times 10^{5}<4$.8. As for the $Y_{2, i j}^{d}$ couplings, we find $Y_{2, s s}^{d}=0.1 \pm 0.1, Y_{2, s b}^{d}=0.004 \pm 0.005$ and $Y_{2, b b}^{d}=0.017 \pm 0.005$ assuming Gaussian distributions. In particular, both $Y_{2, s s}^{d}$ and $Y_{2, s b}^{d}$ flip their signs for the positive solutions of $Y_{2, t c}^{u}$ whereas $Y_{2, b b}^{d}$ remains unaffected.

Finally, in the bottom right panel of figure 8 we observe that the preferred values for the charged Higgs mass are of order $3 \mathrm{TeV}$ with $\tan \beta \approx 1$. We find that the combined contribution of FCNC likelihoods fits better the data for this particular mass range. Similarly, although values of $\tan \beta$ up to 50 are possible in the GTHDM when using theoretical constraints alone, we identified that once we take into account all flavour constraints, there is a clear preference for low values, close to $\tan \beta \approx 1$, in agreement with $[14,184,185]$. This preference can be understood as follows. The box contributions in figures $2 \mathrm{a}-2 \mathrm{~b}$ depend on the Green function $\mathcal{B}^{H(0)}$ in eq. (3.17), which for values of the charged Higgs mass $m_{H^{ \pm}}<2 \mathrm{TeV}$ or $m_{H^{ \pm}}>4 \mathrm{TeV}$ significantly over- or undershoot, respectively, the observed value of $\Delta C_{9} \approx-1$ (see below). Furthermore, the measurement of the $B_{c}$ lifetime and the BaBar collaboration $B \rightarrow D^{(\star)} \tau \bar{\nu}$ distributions, both of which depend strongly on $\tan \beta$ and $m_{H^{ \pm}}$through the $C_{R, L}^{c b}$ in eq. (3.28), push both $\tan \beta$ and $m_{H^{ \pm}}$to values lower than 2 and greater than $2 \mathrm{TeV}$ respectively. In addition to this, we have also noticed a strong penalty for large $\tan \beta$ values coming from the $B_{s} \rightarrow \mu^{+} \mu^{-}$decays, which is due to the strong $\tan \beta$ dependence on the $C_{10}$ and (pseudo) scalar WCs. Lastly the preferred masses of the other heavy Higgses, $m_{H}$ and $m_{A}$, are of the same order as $m_{H^{ \pm}}$as was expected because of the oblique parameter constraints. The best fit values for some relevant scan parameters can be found in table 5 .

\footnotetext{
${ }^{9}$ We first found those two regions of solutions via an auxiliary scanning method based on the quadratic approximation to $\chi^{2}$ as a function of the WCs (see appendix C).
} 


\begin{tabular}{|c|c|}
\hline Parameter & Best fit \\
\hline$m_{H, A}$ & $3485 \mathrm{GeV}$ \\
\hline$m_{H^{ \pm}}$ & $3429 \mathrm{GeV}$ \\
\hline$m_{12}$ & $2426 \mathrm{GeV}$ \\
\hline $\tan \beta$ & 0.98 \\
\hline$Y_{2, t t}^{u}$ & 0.60 \\
\hline$Y_{2, c c}^{u}$ & 1.15 \\
\hline$Y_{2, t c}^{u}$ & -0.64 \\
\hline$Y_{2, b b}^{d}$ & 0.017 \\
\hline$Y_{2, s s}^{d}$ & 0.10 \\
\hline$Y_{2, s b}^{d}$ & 0.004 \\
\hline$Y_{2, \mu \mu}^{l}$ & -0.04 \\
\hline$Y_{2, \tau \tau}^{l}$ & -0.36 \\
\hline$Y_{2, \mu \tau}^{l}$ & 0.75 \\
\hline
\end{tabular}

\begin{tabular}{|c|c|}
\hline Wilson coefficient & Best fit \\
\hline $\operatorname{Re}\left(\Delta C_{Q_{1}}\right)$ & $0.14 \pm 0.01$ \\
\hline $\operatorname{Re}\left(\Delta C_{2}\right)$ & $-0.018 \pm 0.005$ \\
\hline $\operatorname{Re}\left(\Delta C_{7}\right)$ & $0.002 \pm 0.01$ \\
\hline $\operatorname{Re}\left(\Delta C_{7}^{\prime}\right)$ & $0.01 \pm 0.01$ \\
\hline $\operatorname{Re}\left(\Delta C_{8}\right)$ & $0.002 \pm 0.015$ \\
\hline $\operatorname{Re}\left(\Delta C_{8}^{\prime}\right)$ & $0.01 \pm 0.01$ \\
\hline $\operatorname{Re}\left(\Delta C_{9}\right)$ & $-0.89 \pm 0.15$ \\
\hline $\operatorname{Re}\left(\Delta C_{10}\right)$ & $-0.19 \pm 0.14$ \\
\hline
\end{tabular}

Table 5. Best fit values for the scan parameters (left) and WCs for $b \rightarrow s \mu^{+} \mu^{-}$transitions (right). We show only $\operatorname{Re}\left(\Delta C_{Q_{1}}\right)$ given that at tree level and in the alignment limit $\operatorname{Re}\left(\Delta C_{Q_{1}}\right)=\operatorname{Re}\left(\Delta C_{Q_{2}}\right)$ and $m_{s} / m_{b} \operatorname{Re}\left(\Delta C_{Q_{1}}\right)=\operatorname{Re}\left(\Delta C_{Q_{1}}^{\prime}\right)=-\operatorname{Re}\left(\Delta C_{Q_{2}}^{\prime}\right)$. The uncertainties on the WCs were computed with GAMBIT assuming a symmetric Gaussian distribution from the resulting one-dimensional profile likelihoods. We do not display the $\operatorname{Re}\left(\Delta C_{9,10}^{\prime}\right)$ WCs either which we find to be suppressed by a factor of $m_{b} / m_{t}$ compared to their non prime counterparts.

\subsection{Neutral and charged anomalies}

In table 5 we show the best fit values for the parameters from the scans (left) and the muon specific WCs evaluated at the best fit point (right), where in particular, $\Delta C_{9}$ is consistent with the value obtained by model independent fits at the $1 \sigma$ level. In this sense, the neutral anomalies can indeed be explained in the GTHDM as shown in figure 9. Furthermore, coming from the quadratic dependence in the branching ratio $\operatorname{BR}\left(B_{s} \rightarrow \mu^{+} \mu^{-}\right)$, we can see two regions of solutions for the scalar WC $\Delta C_{Q_{1}}$, one of them containing the SM prediction within $2 \sigma$. In addition, we ran a complementary scan invalidating points for $\left|\Delta C_{Q_{1}}\right|>0.1$ and found that the corresponding region of solutions gives an equally good fit to the data, i.e., the preference over the second region of solutions is completely arbitrary.

In order to better understand the contribution of the GTHDM to the various rates and angular observables, we display various plots comparing both the SM and the GTHDM predictions along the experimental data. For the angular observables $\left\langle P_{1}\right\rangle$ and $\left\langle P_{5}^{\prime}\right\rangle$ defined in eqs. (4.4) and (4.5), we show in figure 10 their predictions compared to the CMS 2017 [186], ATLAS 2018 [187] and LHCb 2020 [188] data. For $\left\langle P_{1}\right\rangle$ (figure 10 left) the GTHDM distribution is rather indistinguishable from the $\mathrm{SM}$ one, except in the $[1,2] \mathrm{GeV}^{2}$ bin close to the photon pole and sensitive to $C_{7}^{(\prime)}$. The situation is different for $\left\langle P_{5}^{\prime}\right\rangle$ (figure 10 right) in which the GTHDM prediction fits the LHCb 2020 data better, particularly in the $C_{7}^{(\prime)}$ - 

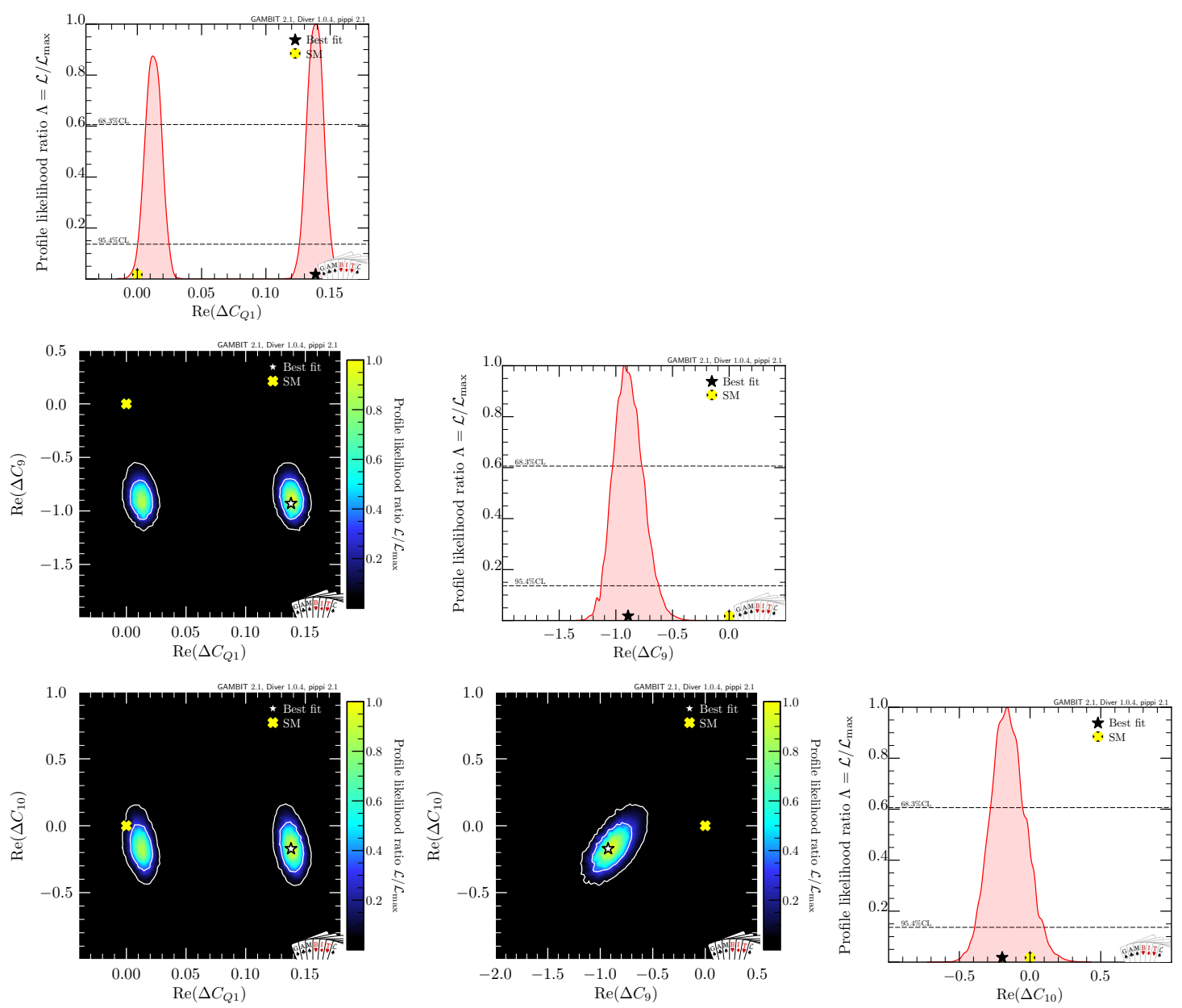

Figure 9. One- and two-dimensional profile likelihoods for three of the Wilson coefficients computed from the fit.

$C_{9}^{(\prime)}$ interference region $\left(1<q^{2}<6 \mathrm{GeV}^{2}\right)$. We also provide in figure 11 predictions for the angular observables in the $S_{i}$ basis using the same LHCb 2020 measurements and also the ATLAS 2018 [187] data. We can see that the GTHDM fits better the LHCb data [188] in the large recoil region than the SM by $2 \sigma$. We also note that neither the SM or the GTHDM can explain the central values (with larger uncertainties) from the ATLAS 2018 data.

As for the measured branching ratios of $B^{0} \rightarrow K^{0 *} \mu^{+} \mu^{-}$and $B^{+} \rightarrow K^{+} \mu^{+} \mu^{-}$, in figure 12 we show the SM and GTHDM predictions using the LHCb results [189-191], where we can see again how the GTHDM fits better the data compared to the SM, specially in the region above the open charm threshold, sensitive to both $C_{9}^{(\prime)}$ and $C_{10}^{(\prime)}$. In contrast, the performance of the model is worse than the SM (figure 13 left) in the low recoil region of the differential branching ratio $\frac{d \mathrm{BR}}{d q^{2}}\left(\Lambda_{b} \rightarrow \Lambda \mu^{+} \mu^{-}\right)$when comparing to the LHCb 2015 [192] data. As pointed out in [65], the decays of the $\Lambda_{b}$ baryon, such as $\Lambda_{b} \rightarrow \Lambda \mu^{+} \mu^{-}$have much larger uncertainties than those of the corresponding meson decays. However, once more experimental data is available, recent [193] and future developments of lattice calculations would eventually make this decay providing similar constraints as other $b \rightarrow s \mu^{+} \mu^{-}$ 

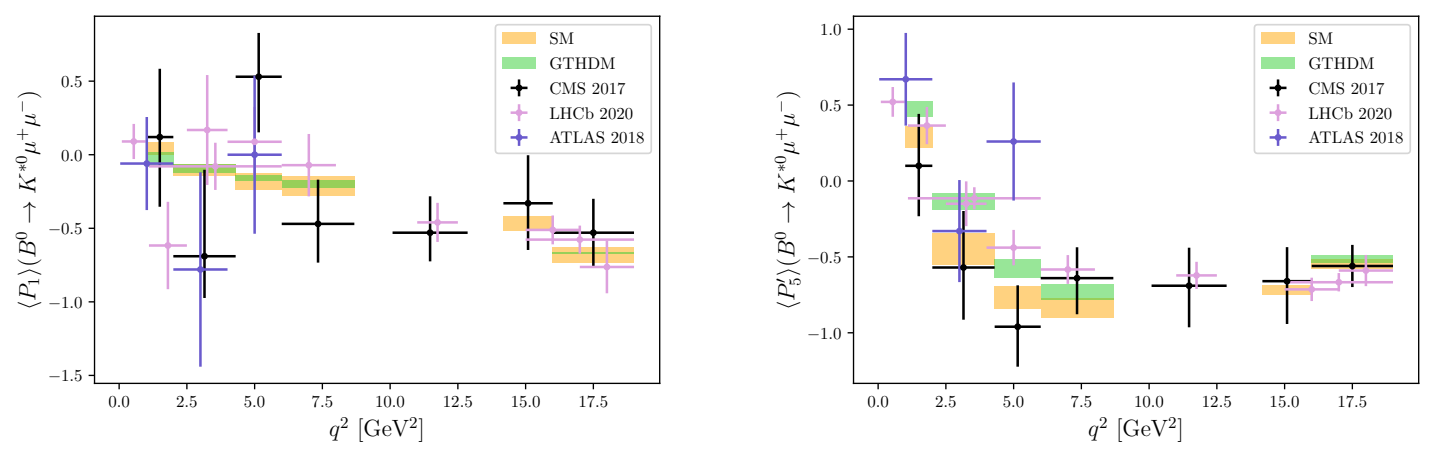

Figure 10. Predicted distributions for Left: $\left\langle P_{1}\right\rangle$ and Right: $\left\langle P_{5}^{\prime}\right\rangle$ compared to the CMS 2017 [186], ATLAS 2018 [187] and LHCb 2020 [188] data. The theoretical uncertainties using GAMBIT have been computed assuming a symmetric Gaussian distribution for the resulting one-dimensional profile likelihoods for each one of the bins. The theory predictions close to the $J / \psi(1 S)$ and $\psi(2 S)$ narrow charmonium resonances are vetoed from all our plots.
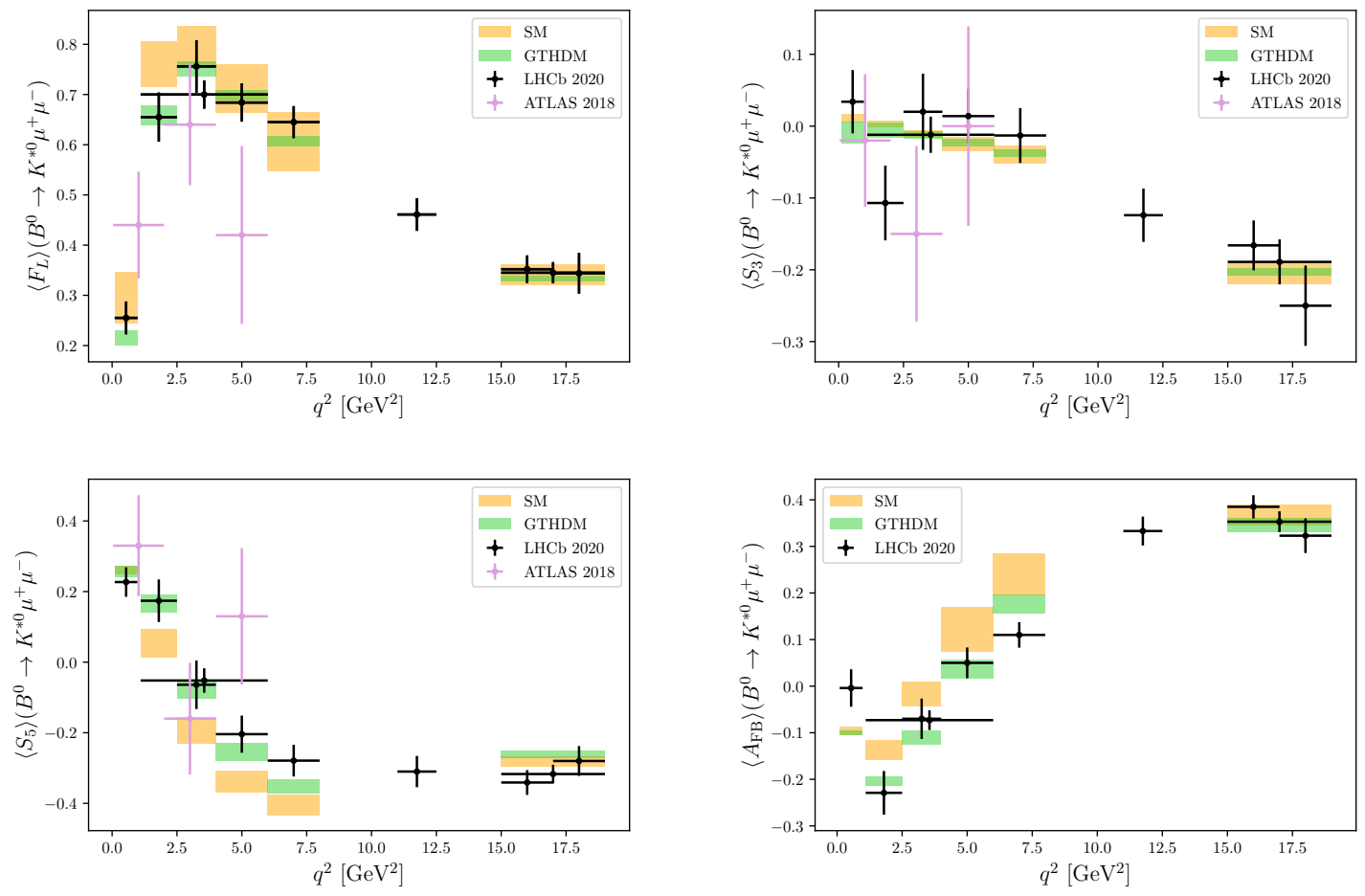

Figure 11. Predicted distributions for the form factor dependent observables in the $S_{i}$ basis using both the ATLAS 2018 [187] and the LHCb 2020 [188] data. 

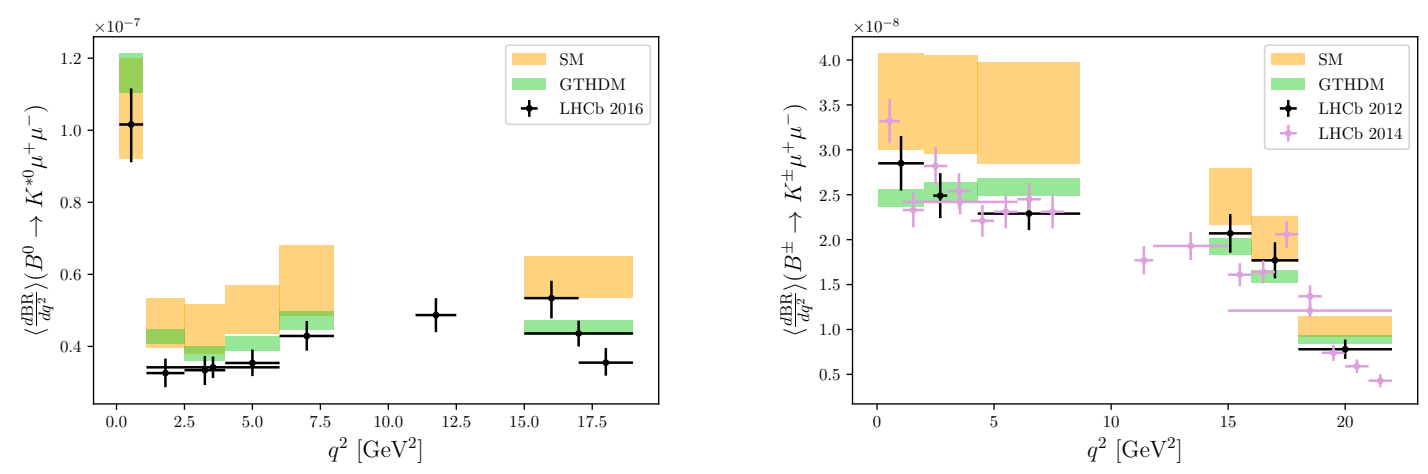

Figure 12. Left: differential branching ratio for $\frac{d \mathrm{BR}}{d q^{2}}\left(B^{0} \rightarrow K^{* 0} \mu^{+} \mu^{-}\right)$with the LHCb 2016 data [189]. Right: $\frac{d \mathrm{BR}}{d q^{2}}\left(B^{+} \rightarrow K^{+} \mu^{+} \mu^{-}\right)$compared to the LHCb 2012 and 2014 measurements [190, 191].
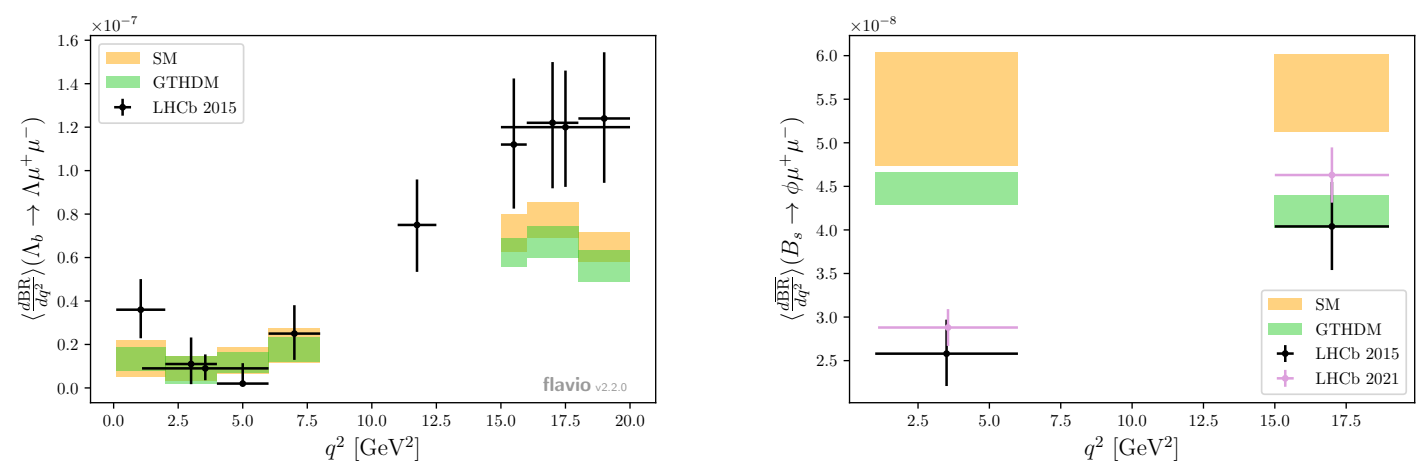

Figure 13. Left: differential branching ratio $\frac{d \mathrm{BR}}{d q^{2}}\left(\Lambda_{b} \rightarrow \Lambda \mu^{+} \mu^{-}\right)$obtained with flavio [170] compared to the LHCb 2015 [192] data. Right: $\frac{d \mathrm{BR}}{d q^{2}}\left(B_{s} \rightarrow \phi \mu^{+} \mu^{-}\right)$compared to the LHCb 2015 and 2021 data $[194,195]$.

transitions. Finally, the results for the $\frac{d \mathrm{BR}}{d q^{2}}\left(B_{s} \rightarrow \phi \mu^{+} \mu^{-}\right)$distribution are shown in figure 13 right. The large recoil region of the experimental data deviates from both the SM and GTHDM predictions by approximately $3 \sigma$, and for the low recoil bin the GTHDM performs slightly better than the SM by approximately $1 \sigma$.

Last but not least important observables related to the $b \rightarrow s \mu^{+} \mu^{-}$transitions are the ratios $R\left(K^{(*)}\right)$. Despite being only three bins in total [150, 152, 196], these measurements have been intensively studied as they provide evidence for LFU violation. We include in our fit the latest LHCb collaboration data for the $R\left(K^{*}\right)$ and $R(K)$ ratios from 2021 [150] and 2017 [152] respectively and obtain the plots in figure 14, where we compare also to the Belle 2019 experiment data $[197,198]$. The effect from the fit on the $R\left(K^{(*)}\right)$ ratios is significant, explaining the LHCb 2021 measurement of $R(K)$ at the $1 \sigma$ level.

The next interesting results are related with the charged anomalies, in particular we find that the $R\left(D^{(*)}\right)$ ratio can (can not) be explained at the $1 \sigma$ level with the GTHDM, a result in agreement with the phenomenological analysis of [132]. We furthermore corrobo- 

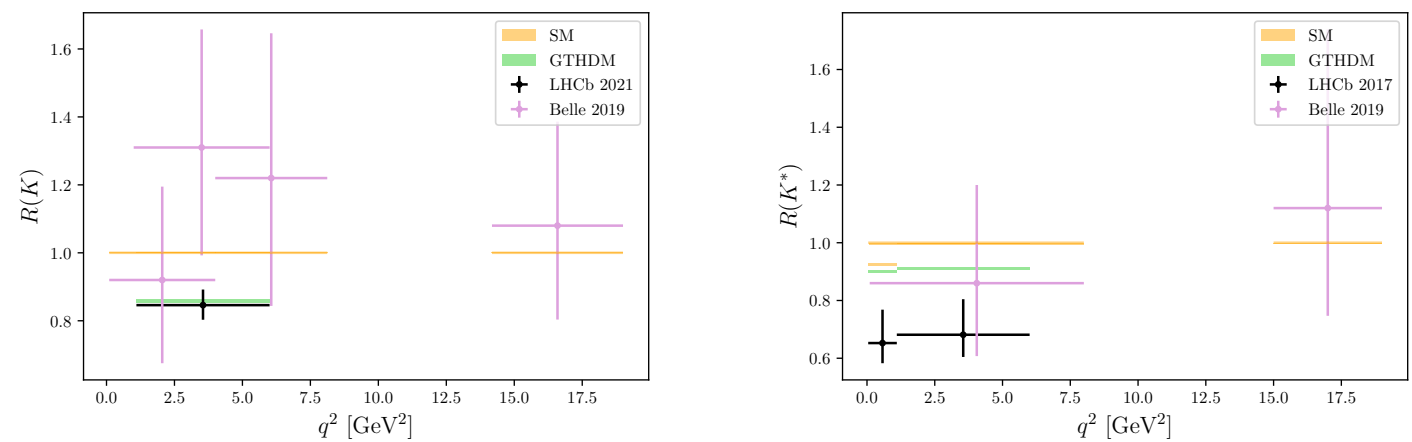

Figure 14. $R\left(K^{(*)}\right)$ theoretical ratios compared to both the LHCb $[150,152]$ and Belle data [197, 198].

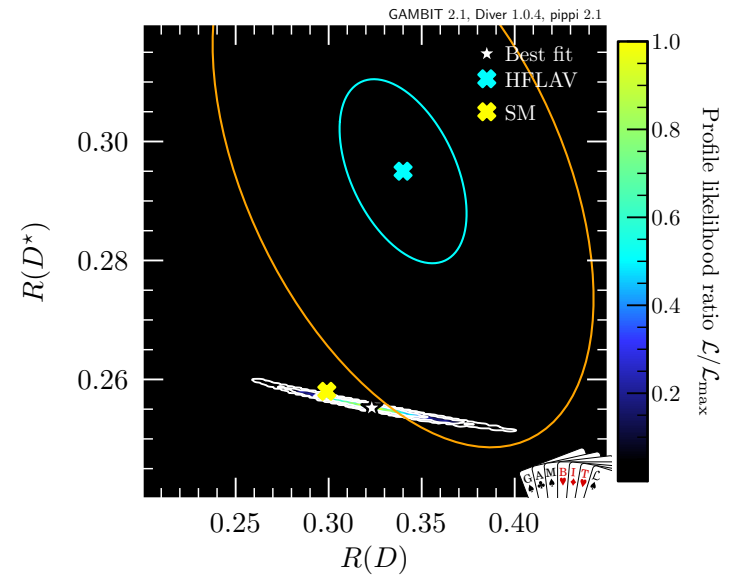

Figure 15. $R\left(D^{*}\right)$ versus $R(D)$ correlated ratios. The cyan and orange lines are the $1 \sigma$ and $3 \sigma$ deviations from the HFLAV average respectively.

rate that the constraint coming from the $B_{c}$ lifetime makes it very difficult to fit $R\left(D^{*}\right)$ and $R(D)$ simultaneously. In figure 15 we show the preferred values by the profile likelihood. We see a slightly better performance of the GTHDM compared to the SM with respect to the HFLAV average. Regarding the $d \Gamma\left(B \rightarrow D^{(\star)} \tau \bar{\nu}\right) /\left(\Gamma d q^{2}\right)$ distributions measured by BaBar [168], we find that the GTHDM prediction is indistinguishable from the SM, in agreement with [16]. We find furthermore that the longitudinal polarisation $F_{L}\left(D^{*}\right)$ is strongly correlated with $R\left(D^{*}\right)$ and the model is not able to explain the Belle measurement, giving a best fit value of $0.458 \pm 0.006$.

\subsection{Anomalous $(g-2)_{\mu}$}

With regards to the anomalous magnetic moment of the muon, $(g-2)_{\mu}$, we find that a simultaneous explanation using all the likelihoods defined before is not possible (solid red line in figure 16). However, when doing a fit to all other observables except the neutral anomalies, i.e., without using the HEPLike likelihoods, the model is able to explain the 


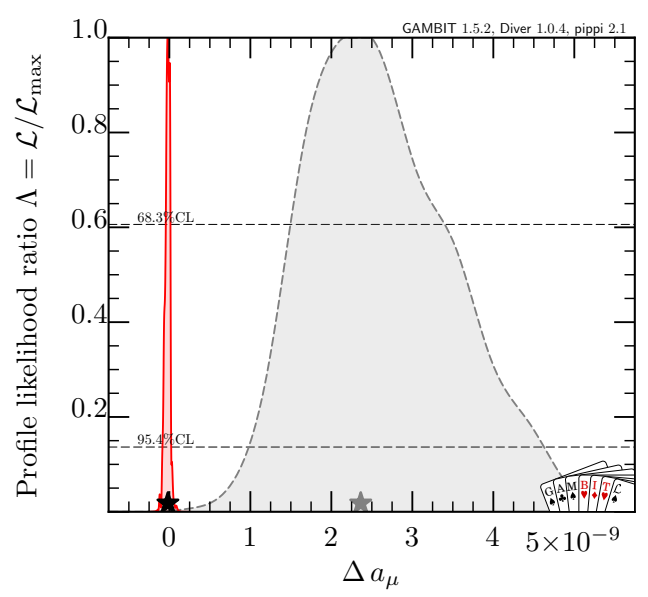

Figure 16. One-dimensional profile likelihood for $\Delta a_{\mu}$. The solid red line shows the result from the fit using all likelihoods and observables defined in this study. The dashed gray line is obtained using all but the HEPLike likelihoods instead.

measured $\Delta a_{\mu}$ by Fermilab at the $1 \sigma$ level (dashed gray line in figure 16). Furthermore, when evaluating the performance of the HEPLike likelihoods for the best fit value, we find a SM-like behavior with all NP WCs close to zero, except for those scalar WCs that enter in $\operatorname{BR}\left(B_{s} \rightarrow \mu^{+} \mu^{-}\right)$.

\subsection{Projections for future and planned experiments}

Although a detailed collider analysis is beyond the scope of the present work, we have included as pure observables the branching ratio for $t \rightarrow c h$ and $h \rightarrow b s^{10}$ at tree level. These tree level branching ratios in the GTHDM are suppressed as $c_{\beta \alpha}^{2}\left|\xi_{t c(b s)}^{u(d)}\right|^{2}$, respectively, so that in the alignment limit they will be exactly zero. In order to study the effects of this fined tuned suppression, we have ran a second scan with $s_{\beta \alpha} \in[0.9999,1]$ and we found the branching ratio of $t \rightarrow c h$ decays are of order $10^{-11}-10^{-7}$, which although are outside future searches sensitivities, they are larger than the SM loop prediction $\left(\sim 10^{-15}\right)$ and well below the current experimental upper bound obtained by the ATLAS collaboration [199]

$$
\mathrm{BR}(t \rightarrow c h)<1.1 \cdot 10^{-3} .
$$

Concerning the $\mathrm{BR}(h \rightarrow b s)$ observable, it was shown in [71] that it is related to tree level $B_{s}-\bar{B}_{s}$ oscillations which are not only proportional to $c_{\beta \alpha}^{2}$ but also to pseudoscalar contributions independent of the scalar CP-even mixing. Hence, in figure 17 we see that $h \rightarrow b s$ is not as constrained as $t \rightarrow c h$ with values ranging from $10^{-7}$ up to $10^{-3}$ at the $1 \sigma$ level, which may be within range of the ILC [200].

Regarding LFV searches, we show in figure 18 the profile likelihood for the $\tau \rightarrow 3 \mu$ and $\tau \rightarrow \mu \gamma$ branching ratios. We see that the best fit value for the $\tau \rightarrow 3 \mu$ decay is well within the projected sensitivity in the Belle II experiment [201] with a discovery

\footnotetext{
${ }^{10}$ We are not aware of current bounds for the $h \rightarrow b s$ branching ratio so we did not define an associated likelihood function for it.
} 


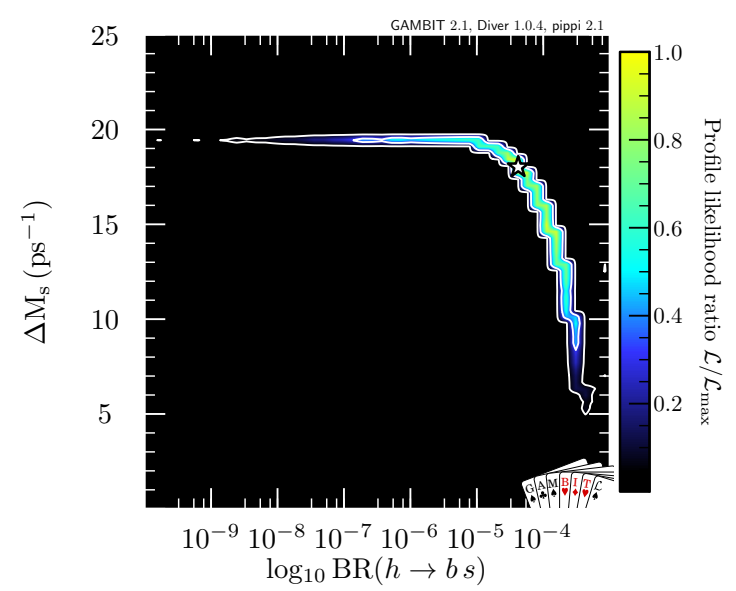

Figure 17. Profile likelihood contours in the $\Delta M_{s}-\mathrm{BR}(h \rightarrow b s)$ plane obtained with a scan using $s_{\beta \alpha} \in[0.9999,1]$. The observed correlation is expected from eq. (4.18) in [71].

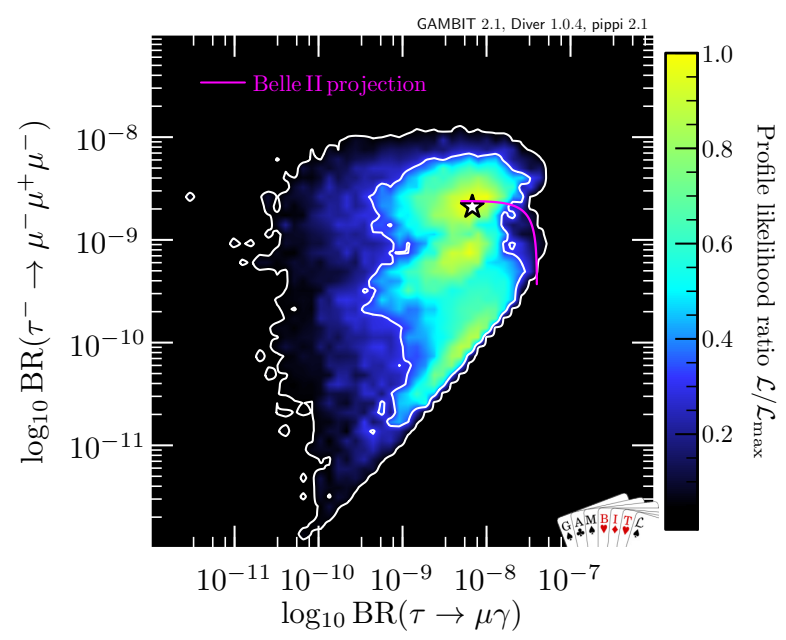

Figure 18. $\operatorname{BR}(\tau \rightarrow 3 \mu)$ versus $\operatorname{BR}(\tau \rightarrow \mu \gamma)$. The magenta solid line is the combined Belle II experiment future sensitivity obtained for both observables using a one-sided Gaussian upper limit likelihood function at 90\%C.L.

potential for $\operatorname{BR}(\tau \rightarrow 3 \mu) \sim 10^{-9}$. Regarding the $\tau \rightarrow \mu \gamma$ decay, we find that with the projected future sensitivity, the GTHDM prediction could be confirmed with values for the branching ratio varying from $10^{-9}$ up to $10^{-8}$. As mentioned earlier, the $\tau \rightarrow 3 \mu$ decay receives contributions in the GTHDM from all tree, dipole and contact terms, in such a way that a possible detection in the $\tau \rightarrow \mu \gamma$ channel will not necessarily imply a strong constraint for $\operatorname{BR}(\tau \rightarrow 3 \mu)$.

With respect to $h \rightarrow \tau \mu$, with the model best fit point values, we computed the branching ratio $\mathrm{BR}(h \rightarrow \tau \mu)$ obtaining values from $10^{-2}$ down to $10^{-6}$ which are within the future sensitivity at the HL-LHC, reaching the $0.05 \%$ limit [202]. 
Finally, as for the $B_{s} \rightarrow \tau^{+} \tau^{-}$decay, we find values of at most $\operatorname{BR}\left(B_{s} \rightarrow \tau^{+} \tau^{-}\right) \sim$ $\mathcal{O}\left(10^{-6}\right)$ with our best fit point, which is one order of magnitude higher than the SM prediction, but out of reach of the future sensitivity in both the HL-LHC and the Belle-II experiments with limits at $\mathcal{O}\left(10^{-4}\right)[201,203]$. Regarding the branching ratio $\mathrm{BR}\left(B^{+} \rightarrow\right.$ $\left.K^{+} \tau^{+} \tau^{-}\right)$, as in the $B_{s} \rightarrow \tau^{+} \tau^{-}$decay, the predicted branching ratio $\operatorname{BR}\left(B^{+} \rightarrow K^{+} \tau^{+} \tau^{-}\right)$ is of order $10^{-7}-10^{-6}$, out of reach for Belle-II projections at $2 \times 10^{-5}$.

\section{Conclusions and outlook}

We presented a frequentist inspired likelihood analysis for the GTHDM including the charged anomalies, $b \rightarrow s \mu^{+} \mu^{-}$transitions and the anomalous magnetic moment of the muon along with other flavour observables. The analysis was carried out using the open source global fitting framework GAMBIT. We computed the GTHDM WCs and validated them obtaining full agreement with the one loop calculations reported in the literature after the different notation factors were taken into account. As expected, we found that the GTHDM can explain the neutral anomalies at the $1 \sigma$ level. Additionally, we also confirmed that the model is able to fit the current experimental values of the $R(D)$ ratio at the $1 \sigma$ level, but it can not accommodate the $D^{*}$ charmed meson observables $R\left(D^{*}\right)$ and $F_{L}\left(D^{*}\right)$. Furthermore, we inspected the fitted values for the angular observables in $b \rightarrow s \mu^{+} \mu^{-}$transitions, obtaining in general a better performance with the GTHDM in comparison to the SM.

Then, based on the obtained best fit values of the model parameters and their $1 \sigma$ and $2 \sigma$ C.L. regions, we made predictions impacting directly in the future collider observables $\mathrm{BR}(t \rightarrow c h), \mathrm{BR}(h \rightarrow b s), \mathrm{BR}(h \rightarrow \tau \mu), \mathrm{BR}\left(B_{s} \rightarrow \tau^{+} \tau^{-}\right), \mathrm{BR}\left(B^{+} \rightarrow K^{+} \tau^{+} \tau^{-}\right)$and the flavour violating decays of the $\tau$ lepton, $\operatorname{BR}(\tau \rightarrow 3 \mu)$ and $\operatorname{BR}(\tau \rightarrow \mu \gamma)$. We find that the model predicts values of $\mathrm{BR}(t \rightarrow c h), \mathrm{BR}\left(B_{s} \rightarrow \tau^{+} \tau^{-}\right)$and $\mathrm{BR}\left(B^{+} \rightarrow K^{+} \tau^{+} \tau^{-}\right)$that are out of reach of future experiments, but its predictions for $\mathrm{BR}(h \rightarrow b s)$ and $\mathrm{BR}(h \rightarrow \tau \mu)$ are within the future sensitivity of the HL-LHC or the ILC. We also find that the predictions for the $\tau \rightarrow 3 \mu$ and $\tau \rightarrow \mu \gamma$ decays are well within the projected limits of the Belle II experiment. In summary, the next generation of particle colliders will have the sensitivity to probe, discover or exclude large parts of the parameter space of the GTHDM, and thus it serves as a further motivation for the development of higher energy and higher intensity particle colliders.

We can envision many avenues of future investigation using the tools and techniques developed for this work. The complete parameter space of the GTHDM is enormous, and thus for this study we have only focused on a subset of CP-conserving interactions between second and third generation fermions. The inclusion of the first generation in the Yukawa textures would introduce additional interactions and decay channels, possibly improving the fit to various of the flavour anomalies, while at the same time introducing new relevant constraints, such as rare kaon decays, e.g. from the NA62 experiment, and LFV muon decays, e.g. from the Mu2e experiment. CP-violation in kaon and $B$-meson decays would also become important constraints in case of complex Yukawa textures. Modifications of the GTHDM may also lead to improved fits to some flavour observables, for instance it has been shown that with the addition of right-handed neutrinos the model can better accom- 
modate the neutral anomalies. Lastly, in this study we have not included detailed collider constraints from, e.g., searches for heavy Higgs bosons at colliders. Such a detailed study is a clear follow up from this work and it will showcase the complementarity of flavour and collider searches to constrain models of new physics that tools such as GAMBIT can explore.

Finally, in view of the latest experimental measurement made by the Fermilab Muon $g-2$ Collaboration, we performed a simultaneous fit to $\Delta a_{\mu}$ constrained by the charged anomalies finding solutions at the $1 \sigma$ level. Once the neutral anomalies are included, however, a simultaneous explanation is unfeasible. A detailed study looking for a simultaneous explanation of both $g-2$ and the neutral anomalies in the GTHDM will be presented in a follow-up work.

\section{Acknowledgments}

We thank Martin White, Filip Rajec and the rest of the GAMBIT community for their suggestions and advice. We would also like to thank Dominik Stöckinger and Hyejung Stöckinger-Kim for their help and guidance on the dominant contributions to muon $g-2$. C.S. thanks Ulrik Egede for useful comments on the future sensitivity of the HL-LHC, and Peter Stangl for discussions about correlations in FCNC observables. The work of C.S. was supported by the Monash Graduate Scholarship (MGS) and the Monash International Tuition Scholarship (MITS). T.E.G. is supported by DFG Emmy Noether Grant No. KA 4662/1-1. The research placement of D.J. for this work was supported by the Australian Government Research Training Program (RTP) Scholarship and the Deutscher Akademischer Austauschdienst (DAAD) One-Year Research Grant. The work of P.A. was supported by the Australian Research Council Future Fellowship grant FT160100274. The work of P.A., C.B. and T.E.G. was also supported with the Australian Research Council Discovery Project grant DP180102209. The work of C.B. was supported by the Australian Research Council through the ARC Centre of Excellence for Particle Physics at the Terascale CE110001104. This project was also undertaken with the assistance of resources and services from the National Computational Infrastructure, which is supported by the Australian Government. We thank Astronomy Australia Limited for financial support of computing resources, and the Astronomy Supercomputer Time Allocation Committee for its generous grant of computing time.

\section{A Gauge dependent term}

The box diagrams in figures $2 \mathrm{c}-2 \mathrm{~d}$ will be gauge dependent, in the Feynman gauge and with $H=m_{H^{ \pm}}^{2} / m_{W}^{2}$ we get,

$$
\mathcal{B}_{\text {mix }}^{H(0)}(t, H)=\frac{\left[G_{1}(t, H)+V_{t s}^{*} G_{2}(t, H)\right]}{(H-1)^{2} \sqrt{H}(t-1)^{2} \sqrt{t}(H t-1)^{2}},
$$


where

$$
\begin{aligned}
G_{1}(t, H)= & V_{t b} V_{c s}^{*} H \xi_{c t}^{u}(t-1) t B_{1}(t, H), \\
G_{2}(t, H)= & V_{t b}\left[H \xi_{t t}^{u}(t-1) t B_{1}(t, H)-\xi_{t t}^{u *}(H t-1) B_{2}(t, H)\right] \\
& -V_{c b} \xi_{c t}^{u *}(H t-1) B_{2}(t, H),
\end{aligned}
$$

with

$$
\begin{aligned}
B_{1}(t, H)= & (H t-1)^{2} \log H-(H-1)[(t-1)(H t-1)+(H-1) t \log (H t)], \\
B_{2}(t, H)= & (t-1)^{2}\left[H((H-1) H-1) t-(H-1)^{2}\right] \log H-(H-1) B_{3}(t, H), \\
B_{3}(t, H)= & -\left[H^{2}(t-1)^{2} t+H((t-2)(t-1) t-1)+(t-1) t+1\right] \log t \\
& +(t-1)\left[-H t^{2}+(t-1)(H(H t+t-1)+1) \log (H t)+t\right] .
\end{aligned}
$$

\section{B Loop functions and vertex couplings}

The one-loop contributions can be separated into fermion-fermion-scalar (FFS) and scalarscalar-fermion (SSF) diagrams shown in the diagrams shown in figure 5. As seen in these diagrams, we can have any one of the SM charged leptons or neutrinos paired with any neutral Higgs boson $\phi=h, H, A$ or the charged Higgs boson $H^{ \pm}$respectively. The contributions from each of these diagrams with a scalar-fermion pair from a lepton of generation $a$ to a lepton of generation $b$ are shown below:

$$
\begin{aligned}
A_{a b L}^{(F F S)}(\phi, i)= & \frac{1}{16 \pi^{2} m_{\phi}^{2}}\left(\frac{\Gamma_{\phi i b}^{l *} \Gamma_{\phi i a}^{l}}{24} E\left(\frac{m_{l_{i}}^{2}}{m_{\phi}^{2}}\right)+\frac{m_{l_{b}}}{m_{l_{a}}} \frac{\Gamma_{\phi a i}^{l *} \Gamma_{\phi b i}^{l}}{24} E\left(\frac{m_{l_{i}}^{2}}{m_{\phi}^{2}}\right)\right. \\
& \left.+\frac{m_{l_{i}}}{m_{l_{a}}} \frac{\Gamma_{\phi i b}^{l} \Gamma_{\phi a i}^{l *}}{3} F\left(\frac{m_{l_{i}}^{2}}{m_{\phi}^{2}}\right)\right), \\
A_{a b L}^{(S S F)}\left(H^{ \pm}, i\right)= & \frac{1}{16 \pi^{2} m_{H^{ \pm}}^{2}} \frac{\Gamma_{H^{ \pm} i \Gamma_{H^{ \pm} i a}}^{l *}}{24} B\left(\frac{m_{\nu_{i}}^{2}}{m_{H^{ \pm}}^{2}}\right), \\
\Gamma_{H^{ \pm} b a}^{f} \equiv & \begin{cases}-V_{c a}^{*} \xi_{c b}^{f} & \text { if } f=u, \\
V_{b c} \xi_{c a}^{f} & \text { if } f=d, \\
\xi_{b a}^{f} & \text { if } f=l,\end{cases}
\end{aligned}
$$

where $A_{a b R}=A_{a b L}\left(\Gamma_{i j}^{\phi} \rightarrow \Gamma_{j i}^{\phi *}\right)$.

Additionally, to get the BSM contributions to muon $g-2$, one must subtract of the SM contribution. This contribution is obtained from eq. (B.1), $A_{\mu \mu L, R}^{(F F S)}\left(h_{S M}, \mu\right)$, by using a mass of $m_{h_{S M}}=125.09 \mathrm{GeV}$, and a $\Gamma_{h_{S M}}^{f}$ coupling in eq. (2.24) with $c_{\beta \alpha}=0$ to obtain a SM-like coupling. For muon $g-2$, the dominant BSM contributions at the one-loop level come from the chirality flipping term involves an internal $\tau$ lepton, $m_{\mu} m_{\tau} /\left(48 \pi^{2} m_{\phi}^{2}\right) \Gamma_{\phi \tau \mu}^{\ell} \Gamma_{\phi \mu \tau}^{l *} F\left(m_{\tau}^{2} / m_{\phi}^{2}\right)$, with an enhancement of $m_{\tau}^{2} / m_{\mu}^{2}$. The coupling $\xi_{\tau \mu}^{\ell}$ should be nonzero to get the chirality flip enhancement from the internal $\tau$ lepton in figure 5 . 
The GTHDM contributions to muon $g-2$ from Barr-Zee diagrams with a fermionic loop are given by [34]

$$
\begin{aligned}
A_{\mu \mu}^{(F N)}(\phi, f)= & \frac{\alpha_{E M}^{2} Q_{f} N_{c}^{f}}{4 \pi^{2} m_{W}^{2} s_{W}^{2}} \frac{v^{2}}{m_{\mu}^{2}} \Gamma_{\phi 33}^{f} \Gamma_{\phi 22}^{l}\left(Q_{f} \frac{m_{f}^{2}}{m_{\phi}^{2}} \mathcal{F}_{\phi}\left(m_{\phi}, m_{f}\right)\right. \\
& \left.-\frac{g_{v}^{l} g_{v}^{f}}{4 s_{W}^{2} c_{W}^{2}} \frac{m_{f}^{2}}{m_{\phi}^{2}-m_{Z}^{2}}\left(\mathcal{F}_{\phi}\left(m_{\phi}, m_{f}\right)-\mathcal{F}_{\phi}\left(m_{Z}, m_{f}\right)\right)\right), \\
A_{\mu \mu}^{(F C)}\left(H^{ \pm}, f\right)= & -\frac{\alpha_{E M}^{2} N_{c}^{f} v^{2}}{32 \pi^{2} m_{W}^{2} s_{W}^{4}} \frac{v^{2}}{m_{\mu}^{2}} \frac{m_{f}^{2}}{m_{H^{ \pm}}^{2}-m_{W}^{2}} \Gamma_{A 33}^{f} \Gamma_{A 22}^{l} \\
& \times\left(\mathcal{F}_{H^{ \pm}}^{f}\left(m_{H^{ \pm}}\right)-\mathcal{F}_{W^{ \pm}}^{f}\left(m_{W}\right)\right),
\end{aligned}
$$

where $c_{W}^{2}=\cos ^{2} \theta_{W}, g_{v}^{f}=T_{3 f}-2 Q_{f} s_{W}^{2}$, and $T_{3 f}$ denotes the isospin of the loop fermion $\left(T_{3 f}=(1 / 2,-1 / 2,-1 / 2)\right.$ for $\left.(u, d, l)\right)$. The contributions $A_{\mu \mu}^{(F N)}(\phi, f)$ corresponds to the left diagram of figure 6 , while $A_{\mu \mu}^{(F C)}\left(H^{ \pm}, f\right)$ corresponds to the other two.

The loop function $\mathcal{F}_{\phi}$ used to calculate the two-loop neutral fermionic Barr-Zee contributions to muon g-2 are defined as [34]:

$$
\mathcal{F}(M, m)= \begin{cases}-2+\log \left(\frac{M^{2}}{m^{2}}\right)-\frac{M^{2}-2 m^{2}}{M^{2}} \frac{\Phi(M, m, m)}{M^{2}-4 m^{2}}, & \phi=h, H, \\ -\frac{\Phi(M, m, m)}{M^{2}-4 m^{2}}, & \phi=A,\end{cases}
$$

and the loop functions $\mathcal{F}_{H^{ \pm}}^{f}, \mathcal{F}_{W^{ \pm}}^{f}$ for the charged fermionic Barr-Zee contributions are defined as

$$
\begin{aligned}
\mathcal{F}_{H^{ \pm}}^{l}(M)= & x_{l}+x_{l}\left(x_{l}-1\right)\left(\operatorname{Li}_{2}\left(1-1 / x_{l}\right)-\frac{\pi^{2}}{6}\right)+\left(x_{l}-\frac{1}{2}\right) \log \left(x_{l}\right) \\
\mathcal{F}_{H^{ \pm}}^{d}(M)= & -\left(x_{u}-x_{d}\right)+\left(\frac{\bar{c}}{y}-\frac{c\left(x_{u}-x_{d}\right)}{y}\right) \Phi\left(\sqrt{x_{d}}, \sqrt{x_{u}}, 1\right) \\
& +c\left(\operatorname{Li}_{2}\left(1-x_{d} / x_{u}\right)-\frac{1}{2} \log \left(x_{u}\right) \log \left(x_{d} / x_{u}\right)\right) \\
& +\left(s+x_{d}\right) \log \left(x_{d}\right)+\left(s-x_{u}\right) \log \left(x_{u}\right) \\
\mathcal{F}_{H^{ \pm}}^{d}(M)= & -\left(x_{u}-x_{d}\right)+\left(\frac{\bar{c}}{y}-\frac{c\left(x_{u}-x_{d}\right)}{y}\right) \Phi\left(\sqrt{x_{d}}, \sqrt{x_{u}}, 1\right) \\
& +c\left(\operatorname{Li}_{2}\left(1-x_{d} / x_{u}\right)-\frac{1}{2} \log \left(x_{u}\right) \log \left(x_{d} / x_{u}\right)\right) \\
& +\left(s+x_{d}\right) \log \left(x_{d}\right)+\left(s-x_{u}\right) \log \left(x_{u}\right)-\frac{4}{3} \frac{x_{u}-x_{d}-1}{y} \Phi\left(\sqrt{x_{d}}, \sqrt{x_{u}}, 1\right) \\
& -\frac{\log \left(x_{d}\right)^{2}-\log \left(x_{u}\right)^{2}}{3} \\
c= & \left(x_{u}-x_{d}\right)^{2}-Q_{u} x_{u}+Q_{d} x_{d} \\
\bar{c}= & \left(x_{u}-Q_{u}\right) x_{u}-\left(x_{d}+Q_{d}\right) x_{d} \\
y= & \left(x_{u}-x_{d}\right)^{2}-2\left(x_{u}+x_{d}\right)+1 \\
s= & \left(Q_{u}+Q_{d}\right) / 4
\end{aligned}
$$


where $x_{f}=m_{f}^{2} / M^{2}$, and $\mathcal{F}_{W^{ \pm}}^{f}=\mathcal{F}_{H^{ \pm}}^{f}\left(m_{H^{ \pm}} \rightarrow m_{W}\right)$. The function $\Phi$ needed for the above loop functions is from [204]:

$$
\begin{aligned}
\Phi\left(m_{1}, m_{2}, m_{3}\right)= & \frac{\lambda}{2}\left(2 \log \left(a_{+}\right) \log \left(a_{-}\right)-\log \left(\frac{m_{1}^{2}}{m_{3}^{2}}\right) \log \left(\frac{m_{2}^{2}}{m_{3}^{2}}\right)\right. \\
& \left.-2 \operatorname{Li}_{2}\left(a_{+}\right)-2 \operatorname{Li}_{2}\left(a_{-}\right)+\frac{\pi^{2}}{3}\right) \\
\lambda= & \sqrt{m_{1}^{4}+m_{2}^{4}+m_{3}^{4}-2 m_{1}^{2} m_{2}^{2}-2 m_{2}^{2} m_{3}^{2}-2 m_{3}^{2} m_{1}^{2}} \\
a_{ \pm}= & \frac{m_{3}^{2} \pm m_{1}^{2} \mp m_{2}^{2}-\lambda}{2 m_{3}^{2}}
\end{aligned}
$$

where the masses have been ordered so that $m_{1}<m_{2}<m_{3}$.

The contributions to muon $g-2$ from Barr-Zee diagrams with a bosonic loop are given by [32]

$$
\begin{aligned}
A_{\mu \mu}^{(B H N)}(\phi)= & \frac{\alpha_{E M}}{8 \pi^{3} m_{\phi}^{2}} \frac{v}{m_{\mu}} \operatorname{Re}\left(\Gamma_{\phi \mu \mu}^{l}\right) \lambda_{\phi H^{ \pm} H^{\mp}} \mathcal{A}\left(\frac{m_{H^{ \pm}}^{2}}{m_{\phi}^{2}}\right), \\
A_{\mu \mu}^{(B W N)}(\phi)= & \frac{\alpha_{E M}}{8 \pi^{3} v m_{\mu}} \operatorname{Re}\left(\Gamma_{\phi \mu \mu}^{l}\right) g_{\phi W^{ \pm} W^{\mp}} \mathcal{B}\left(\frac{m_{W}^{2}}{m_{\phi}^{2}}\right), \\
A_{\mu \mu}^{(B H C)}(\phi)= & \frac{\alpha_{E M} \operatorname{Re}\left(\Gamma_{H^{ \pm} \mu \mu}^{l *} \lambda_{\phi H^{ \pm} W^{\mp}}\right)}{64 \pi^{3} s_{w}^{2}\left(m_{H^{ \pm}}^{2}-m_{W}^{2}\right)} \frac{v}{m_{\mu}} \lambda_{\phi H^{ \pm} H^{\mp}} \int_{0}^{1} d x \quad x^{2}(x-1) \\
& \times\left(\mathcal{G}\left(1, \frac{m_{\phi}^{2}}{m_{H^{ \pm}}^{2}}, x\right)-\mathcal{G}\left(\frac{m_{H^{ \pm}}^{2}}{m_{W}^{2}}, \frac{m_{\phi}^{2}}{m_{W}^{2}}, x\right)\right), \\
A_{\mu \mu}^{(B W C)}(\phi)= & \frac{\alpha_{E M} \operatorname{Re}\left(\Gamma_{H^{ \pm}}^{l *} \mu \lambda_{\left.\phi H^{ \pm} W^{\mp}\right)}\right.}{64 \pi^{3} s_{w}^{2} v m_{\mu}\left(m_{H^{ \pm}}^{2}-m_{W}^{2}\right)} g_{\phi W^{ \pm}}{ }^{\mp} \int_{0}^{1} d x \\
& \times\left(\mathcal{G}\left(1, \frac{m_{\phi}^{2}}{m_{H^{ \pm}}^{2}}, x\right)-\mathcal{G}\left(\frac{m_{H^{ \pm}}^{2}}{m_{W}^{2}}, \frac{m_{\phi}^{2}}{m_{W}^{2}}, x\right)\right),
\end{aligned}
$$

where $A_{\mu \mu}^{(B H N)}(\phi), A_{\mu \mu}^{(B H C)}(\phi)$, and $A_{\mu \mu}^{(B W C)}(\phi)$ correspond to figure 7 , and $A_{\mu \mu}^{(B H C)}(\phi)$ to the left panel of figure 7 with $H^{ \pm}$replaced by $W^{ \pm}$.

The couplings $g_{\phi W^{ \pm} W^{\mp}}, \lambda_{\phi H^{ \pm} W^{\mp}}$, and $\lambda_{\phi H^{ \pm} H^{\mp}}$ (between $\phi=h, H, A$ and $H^{ \pm} H^{\mp}$, $\left.H^{ \pm} W^{\mp}, W^{ \pm} W^{\mp}\right)$ can be found by mixing the gauge states in the Lagrangian in eq. (2.16) according to eqs. (2.3), (2.4). Reading off the coefficient of the $\phi-W^{ \pm}-W^{\mp}$ term, where $\phi=h, H, A$, we obtain

$$
g_{\phi W^{ \pm} W^{\mp}}= \begin{cases}\frac{\left(2 m_{H^{ \pm}}^{2}-m_{h}^{2}\right) * \cos (\alpha-3 \beta) * \sin (2 \beta)+\cos (\alpha+\beta) *\left(\left(3 m_{H}^{2}+2 m_{H^{ \pm}}^{2}\right) * \sin (2 \beta)-8 m_{12}^{2}\right)}{8 v^{2} \cos (\beta)^{2} \sin (\beta)^{2}}, & \phi=h, \\ \frac{\left(2 m_{H^{ \pm}}^{2}-m_{H}^{2}\right) * \sin (\alpha-3 \beta)+\left(3 m_{H}^{2}+2 m_{H^{ \pm}}^{2}-4 m_{12}^{2} /(\sin (\beta) \cos (\beta))\right) * \sin (\alpha+\beta)}{2 v^{2} \sin (2 \beta)}, & \phi=H, \\ 0, & \phi=A .\end{cases}
$$

Similarly we can read off the coefficients of the terms involving $\phi-H^{ \pm}-H^{\mp}$ and $\phi-H^{ \pm}-$ 
$W^{\mp}$ terms:

$$
\begin{gathered}
\lambda_{\phi H^{ \pm} H^{\mp}}= \begin{cases}\sin (\alpha-\beta), & \phi=h, \\
\cos (\alpha-\beta), & \phi=H \\
0, & \phi=A,\end{cases} \\
\lambda_{\phi H^{ \pm} W^{\mp}}= \begin{cases}\cos (\alpha-\beta), & \phi=h, \\
-\sin (\alpha-\beta), & \phi=H, \\
-i, & \phi=A .\end{cases}
\end{gathered}
$$

The loop functions used for the two-loop muon $g-2$ bosonic Barr-Zee contributions come from [32] and are defined as one dimensional integrals:

$$
\begin{aligned}
\mathcal{A}(z) & =\frac{1}{2} \int_{0}^{1} d x \frac{x(x-1)}{z-x(1-x)} \log \left(\frac{z}{x(1-x)}\right), \\
\mathcal{B}(z) & =\frac{1}{2} \int_{0}^{1} d x \frac{x * z *(3 x(4 x-1)+10)-x(1-x)}{z-x(1-x)} \log \left(\frac{z}{x(1-x)}\right), \\
\mathcal{G}\left(z_{a}, z_{b}, x\right) & =\frac{1}{x(1-x)-z_{a} * x-z_{b} *(1-x)} \log \left(\frac{z_{a} * x+z_{b} *(1-x)}{x(1-x)}\right) .
\end{aligned}
$$

Similarly, the Barr-Zee fermionic and bosonic contributions to $l \rightarrow l^{\prime} \gamma$ are given by

$$
\begin{aligned}
A_{a b L}^{(2, \mathrm{f})}= & -\sum_{\phi=h, H, A} \sum_{f=t, b, \tau} \frac{N_{c}^{f} Q_{f} \alpha_{E M}}{8 \pi^{3}} \frac{\Gamma_{\phi a b}^{l *}}{m_{l_{a}} m_{f}} \\
& \times\left[\frac{\left(1-4 s_{W}^{2}\right) g_{v}^{f}}{8 s_{W}^{2} c_{W}^{2}}\left\{\operatorname{Re}\left(\Gamma_{\phi 33}^{f}\right) \tilde{F}_{H}\left(\frac{m_{f}^{2}}{m_{\phi}^{2}}, \frac{m_{\phi}^{2}}{m_{Z}^{2}}\right)-i \operatorname{Im}\left(\Gamma_{\phi 33}^{f}\right) \tilde{F}_{A}\left(\frac{m_{f}^{2}}{m_{\phi}^{2}}, \frac{m_{\phi}^{2}}{m_{Z}^{2}}\right)\right\}\right. \\
& \left.+Q_{f}\left\{\operatorname{Re}\left(\Gamma_{\phi 33}^{f}\right) F_{H}\left(\frac{m_{f}^{2}}{m_{\phi}^{2}}\right)-i \operatorname{Im}\left(\Gamma_{\phi 33}^{f}\right) F_{A}\left(\frac{m_{f}^{2}}{m_{\phi}^{2}}\right)\right\}\right], \\
A_{a b L}^{(2, \mathrm{~b})}= & \sum_{\phi=h, H} \frac{\alpha_{E M}}{16 \pi^{3}} \frac{g_{\phi W} W^{\mp} \Gamma_{\phi}^{l *} a b}{m_{l_{a}} v}\left[3 F_{H}\left(\frac{m_{W}^{2}}{m_{\phi}^{2}}\right)+\frac{23}{4} F_{A}\left(\frac{m_{W}^{2}}{m_{\phi}^{2}}\right)+\frac{3}{4} G\left(\frac{m_{W}^{2}}{m_{\phi}^{2}}\right)\right. \\
& +\frac{m_{\phi}^{2}}{2 m_{W}^{2}}\left\{F_{H}\left(\frac{m_{W}^{2}}{m_{\phi}^{2}}\right)-F_{A}\left(\frac{m_{W}^{2}}{m_{\phi}^{2}}\right)\right\}+\frac{1-4 s_{W}^{2}}{8 s_{W}^{2}}\left\{\frac{3}{2}\left\{F_{A}\left(\frac{m_{W}^{2}}{m_{\phi}^{2}}\right)+G\left(\frac{m_{W}^{2}}{m_{\phi}^{2}}\right)\right\}\right. \\
& +\left(5-t_{W}^{2}+\left(1-t_{W}^{2}\right) \frac{m_{\phi}^{2}}{2 m_{W}^{2}}\right) \tilde{F}_{H}\left(\frac{m_{W}^{2}}{m_{\phi}^{2}}, \frac{m_{W}^{2}}{m_{Z}^{2}}\right) \\
& \left.\left.+\left(7-3 t_{W}^{2}-\left(1-t_{W}^{2}\right) \frac{m_{\phi}^{2}}{2 m_{W}^{2}}\right) \tilde{F}_{A}\left(\frac{m_{W}^{2}}{m_{\phi}^{2}}, \frac{m_{W}^{2}}{m_{Z}^{2}}\right)\right\}\right], \\
A_{R}^{(2, \mathrm{f}, \mathrm{b})}= & A_{L}^{(2, \mathrm{f}, \mathrm{b})}\left(\Gamma_{\phi \tau \mu}^{l *} \rightarrow \Gamma_{\phi \mu \tau}^{l}, i \rightarrow-i\right) .
\end{aligned}
$$

These do include the $Z$ boson contributions as per [175]. The coupling $g_{\phi W^{ \pm} W^{\mp}}$ is defined in eq. (B.21), and $t_{W}^{2}=\tan ^{2} \theta_{W}$. 
Finally, the loop functions $F_{H, A}, G$ and $\tilde{F}_{H, A}$ used for $l \rightarrow l^{\prime} \gamma$ flavour-violating processes are defined as

$$
\begin{aligned}
F_{H}(z) & =\frac{z}{2} \int_{0}^{1} d x \frac{1-2 x(1-x)}{x(1-x)-z} \log \frac{x(1-x)}{z} \\
F_{A}(z) & =\frac{z}{2} \int_{0}^{1} d x \frac{1}{x(1-x)-z} \log \frac{x(1-x)}{z}, \\
G(z) & =-\frac{z}{2} \int_{0}^{1} d x \frac{1}{x(1-x)-z}\left[1-\frac{z}{x(1-x)-z} \log \frac{x(1-x)}{z}\right], \\
\tilde{F}_{H}(x, y) & =\frac{x F_{H}(y)-y F_{H}(x)}{x-y} \\
\tilde{F}_{A}(x, y) & =\frac{x F_{A}(y)-y F_{A}(x)}{x-y} .
\end{aligned}
$$

The one-loop contributions to $\tau \rightarrow 3 \mu$ depend on the coefficients $A_{\tau \mu L, R}$ and $g_{i}$ given below [177]:

$$
\begin{aligned}
\mathcal{L}_{\tau \rightarrow 3 \mu}= & -\frac{e m_{\mu}}{2} A_{\tau \mu R}\left(\bar{\tau}_{R} \sigma^{\mu \nu} \mu_{L}\right) F_{\mu \nu}-\frac{e m_{\mu}}{2} A_{\tau \mu L}\left(\bar{\tau}_{L} \sigma^{\mu \nu} \mu_{R}\right) F_{\mu \nu} \\
& -\frac{4 G_{F}}{\sqrt{2}}\left[g_{1}\left(\bar{\mu}_{R} \mu_{L}\right)^{\dagger}\left(\bar{\tau}_{R} \mu_{L}\right)^{\dagger}+g_{2}\left(\bar{\mu}_{L} \mu_{R}\right)^{\dagger}\left(\bar{\tau}_{L} \mu_{R}\right)^{\dagger}\right. \\
& +g_{3}\left(\bar{\mu}_{R} \gamma_{\mu} \mu_{R}\right)^{\dagger}\left(\bar{\tau}_{R} \gamma^{\mu} \mu_{R}\right)^{\dagger}+g_{4}\left(\bar{\mu}_{L} \gamma_{\mu} \mu_{L}\right)^{\dagger}\left(\bar{\tau}_{L} \gamma^{\mu} \mu_{L}\right)^{\dagger} \\
& \left.+g_{5}\left(\bar{\mu}_{L} \gamma_{\mu} \mu_{L}\right)^{\dagger}\left(\bar{\tau}_{R} \gamma^{\mu} \mu_{R}\right)^{\dagger}+g_{6}\left(\bar{\mu}_{R} \gamma_{\mu} \mu_{R}\right)^{\dagger}\left(\bar{\tau}_{L} \gamma^{\mu} \mu_{L}\right)^{\dagger}+\text { h.c. }\right] .
\end{aligned}
$$

In the GTHDM, only $g_{2}$ and $g_{4}$ receive contributions:

$$
\begin{aligned}
& g_{2}=\frac{i m_{\mu}^{2}}{192 \sqrt{2} \pi^{2} G_{F} m_{H^{ \pm}}^{4}} \xi_{\tau \mu}^{l}\left(\left|\xi_{\mu \mu}^{l}\right|^{2}+\left|\xi_{\tau \mu}^{l}\right|^{2}\right)\left(\xi_{\mu \mu}^{l}+\xi_{\tau \tau}^{l}\right), \\
& g_{4}=\frac{-i}{128 \sqrt{2} \pi^{2} G_{F} m_{H^{ \pm}}^{2}} \xi_{\tau \mu}^{l}\left(\left|\xi_{\mu \mu}^{l}\right|^{2}+\left|\xi_{\tau \mu}^{l}\right|^{2}\right)\left(\xi_{\mu \mu}^{l}+\xi_{\tau \tau}^{l}\right) .
\end{aligned}
$$

\section{Auxiliary scanning method}

The two regions of solutions for $Y_{2, t c}^{u}$ were expected already when applying the quadratic approximation to the $\chi^{2}$ function defined in [205] for a fit to the $b \rightarrow s \mu^{+} \mu^{-}$observables solely. Explicitly, the likelihood function is approximated by

$$
\log \mathcal{L}=-\frac{\chi^{2}}{2}, \quad \chi^{2}(\mathbf{C}) \approx \chi_{\min }^{2}+\frac{1}{2}\left(\mathbf{C}-\mathbf{C}_{\mathrm{bf}}\right)^{T} \operatorname{Cov}^{-1}\left(\mathbf{C}-\mathbf{C}_{\mathrm{bf}}\right)
$$

where $\mathbf{C}=\left\{\Delta C_{7}, \Delta C_{9}, \Delta C_{10}, \Delta C_{7}^{\prime}, \Delta C_{9}^{\prime}, \Delta C_{10}^{\prime}\right\}$ are the WCs used as parameters to be fitted, and $\mathrm{Cov}^{-1}$ is the covariance matrix or Hessian obtained using the minuit and 
flavio packages,

$$
\mathrm{Cov}^{-1}=\left(\begin{array}{ccccccc}
5594.96 & -128.83 & 0.1604 & -1156.88 & -0.0139 & -0.0146 \\
-128.83 & 44.89 & -10.11 & -102.95 & -7.153 & -14.66 \\
0.1604 & -10.11 & 34.81 & -90.76 & -6.29 & -12.91 \\
-1156.88 & -102.95 & -90.76 & 3613.3 & -64.07 & -131.44 \\
-0.0139 & -7.153 & -6.29 & -64.07 & 17.34 & -0.037 \\
-0.0146 & -14.66 & -12.91 & -131.44 & -0.037 & 72.17
\end{array}\right),
$$

which encodes a fit using the likelihoods from [65] (excepting the associated likelihoods for the Belle experiment measurements not available in flavio). After obtaining the Hessian, a random generator in Mathematica is requested to find points inside the ellipsoid defined by $\Delta \chi^{2} \leq \sigma_{2 d}(1)$ and $\Delta \chi^{2} \leq \sigma_{2 d}(2)$ for 2 degrees of freedom and boundaries defined by the values of the parameter space in eq. (5.2). With this auxiliary method, we were able to help the Diver sampler to scan over different regions of the parameter space.

Open Access. This article is distributed under the terms of the Creative Commons Attribution License (CC-BY 4.0), which permits any use, distribution and reproduction in any medium, provided the original author(s) and source are credited.

\section{References}

[1] S.P. Martin, A supersymmetry primer, Adv. Ser. Direct. High Energy Phys. 18 (1998) 1 [Adv. Ser. Direct. High Energy Phys. 21 (2010) 1] [hep-ph/9709356] [InSPIRE].

[2] R.D. Peccei and H.R. Quinn, CP conservation in the presence of instantons, Phys. Rev. Lett. 38 (1977) 1440 [INSPIRE].

[3] R.D. Peccei and H.R. Quinn, Constraints imposed by CP conservation in the presence of instantons, Phys. Rev. D 16 (1977) 1791 [INSPIRE].

[4] D. Croon, T.E. Gonzalo, L. Graf, N. Košnik and G. White, GUT physics in the era of the LHC, Front. Phys. 7 (2019) 76 [arXiv:1903.04977] [InSPIRE].

[5] M. Carena, M. Quirós, A. Riotto, I. Vilja and C.E.M. Wagner, Electroweak baryogenesis and low-energy supersymmetry, Nucl. Phys. B 503 (1997) 387 [hep-ph/9702409] [inSPIRE].

[6] J.M. Cline, M. Joyce and K. Kainulainen, Supersymmetric electroweak baryogenesis in the WKB approximation, Phys. Lett. B 417 (1998) 79 [Erratum ibid. 448 (1999) 321] [hep-ph/9708393] [INSPIRE].

[7] T. Konstandin, T. Prokopec, M.G. Schmidt and M. Seco, MSSM electroweak baryogenesis and flavor mixing in transport equations, Nucl. Phys. B 738 (2006) 1 [hep-ph/0505103] [INSPIRE].

[8] V. Cirigliano, M.J. Ramsey-Musolf, S. Tulin and C. Lee, Yukawa and tri-scalar processes in electroweak baryogenesis, Phys. Rev. D 73 (2006) 115009 [hep-ph/0603058] [INSPIRE].

[9] W. Buchmüller, Baryogenesis, dark matter and the maximal temperature of the early universe, Acta Phys. Polon. B 43 (2012) [arXiv:1212.3554] [INSPIRE]. 
[10] D.E. Morrissey and M.J. Ramsey-Musolf, Electroweak baryogenesis, New J. Phys. 14 (2012) 125003 [arXiv: 1206. 2942] [INSPIRE].

[11] T. Konstandin, Quantum transport and electroweak baryogenesis, Phys. Usp. 56 (2013) 747 [Usp. Fiz. Nauk 183 (2013) 785] [arXiv:1302.6713] [INSPIRE].

[12] P. Basler, M. Krause, M. Muhlleitner, J. Wittbrodt and A. Wlotzka, Strong first order electroweak phase transition in the CP-conserving 2HDM revisited, JHEP 02 (2017) 121 [arXiv: 1612.04086] [INSPIRE].

[13] K. Fuyuto, W.-S. Hou and E. Senaha, Electroweak baryogenesis driven by extra top Yukawa couplings, Phys. Lett. B 776 (2018) 402 [arXiv:1705.05034] [INSPIRE].

[14] G.C. Branco, P.M. Ferreira, L. Lavoura, M.N. Rebelo, M. Sher and J.P. Silva, Theory and phenomenology of two-Higgs-doublet models, Phys. Rept. 516 (2012) 1 [arXiv:1106.0034] [INSPIRE].

[15] S. Iguro and Y. Omura, Status of the semileptonic B decays and muon $g-2$ in general $2 H D M s$ with right-handed neutrinos, JHEP 05 (2018) 173 [arXiv: 1802.01732] [INSPIRE].

[16] R. Martinez, C.F. Sierra and G. Valencia, Beyond $\mathcal{R}\left(D^{(*)}\right)$ with the general type-III 2 HDM for $b \rightarrow c \tau \nu$, Phys. Rev. D 98 (2018) 115012 [arXiv: 1805.04098] [InSPIRE].

[17] A. Broggio, E.J. Chun, M. Passera, K.M. Patel and S.K. Vempati, Limiting two-Higgs-doublet models, JHEP 11 (2014) 058 [arXiv:1409.3199] [INSPIRE].

[18] L. Wang and X.-F. Han, A light pseudoscalar of $2 H D M$ confronted with muon $g-2$ and experimental constraints, JHEP 05 (2015) 039 [arXiv:1412.4874] [INSPIRE].

[19] T. Abe, R. Sato and K. Yagyu, Lepton-specific two Higgs doublet model as a solution of muon g- 2 anomaly, JHEP 07 (2015) 064 [arXiv: 1504.07059] [INSPIRE].

[20] E.J. Chun, Z. Kang, M. Takeuchi and Y.-L.S. Tsai, LHC $\tau$-rich tests of lepton-specific $2 H D M$ for $(g-2)_{\mu}$, JHEP 11 (2015) 099 [arXiv: 1507.08067] [INSPIRE].

[21] E.J. Chun, The muon $g-2$ in two-Higgs-doublet models, EPJ Web Conf. 118 (2016) 01006 [Pramana 87 (2016) 41] [arXiv: 1511.05225] [INSPIRE].

[22] E.J. Chun and J. Kim, Leptonic precision test of leptophilic two-Higgs-doublet model, JHEP 07 (2016) 110 [arXiv: 1605.06298] [INSPIRE].

[23] L. Wang, J.M. Yang, M. Zhang and Y. Zhang, Revisiting lepton-specific 2HDM in light of muon g- 2 anomaly, Phys. Lett. B 788 (2019) 519 [arXiv: 1809.05857] [INSPIRE].

[24] E.J. Chun, J. Kim and T. Mondal, Electron EDM and muon anomalous magnetic moment in two-Higgs-doublet models, JHEP 12 (2019) 068 [arXiv:1906.00612] [INSPIRE].

[25] E.J. Chun and T. Mondal, Searching for a light Higgs boson via the Yukawa process at lepton colliders, Phys. Lett. B 802 (2020) 135190 [arXiv: 1909.09515] [INSPIRE].

[26] W.-Y. Keung, D. Marfatia and P.-Y. Tseng, Axion-like particles, two-Higgs-doublet models, leptoquarks, and the electron and muon g-2, LHEP 2021 (2021) 209 [arXiv:2104.03341] [INSPIRE].

[27] P.M. Ferreira, B.L. Gonçalves, F.R. Joaquim and M. Sher, $(g-2)_{\mu}$ in the $2 H D M$ and slightly beyond: an updated view, Phys. Rev. D 104 (2021) 053008 [arXiv:2104.03367] [INSPIRE].

[28] X.-F. Han, T. Li, H.-X. Wang, L. Wang and Y. Zhang, Lepton-specific inert two-Higgs-doublet model confronted with the new results for muon and electron $g-2$ anomalies and multilepton searches at the LHC, Phys. Rev. D 104 (2021) 115001 [arXiv: 2104.03227] [INSPIRE]. 
[29] J.C. Eung and T. Mondal, Leptophilic bosons and muon $g-2$ at lepton colliders, JHEP 07 (2021) 044 [arXiv: 2104.03701] [INSPIRE].

[30] A. Jueid, J. Kim, S. Lee and J. Song, Type-X two-Higgs-doublet model in light of the muon g- 2: confronting Higgs boson and collider data, Phys. Rev. D 104 (2021) 095008 [arXiv:2104.10175] [INSPIRE].

[31] A. Dey, J. Lahiri and B. Mukhopadhyaya, Muon g-2 and a type-X two Higgs doublet scenario: some studies in high-scale validity, arXiv:2106.01449 [INSPIRE].

[32] V. Ilisie, New Barr-Zee contributions to $(g-2)_{\mu}$ in two-Higgs-doublet models, JHEP 04 (2015) 077 [arXiv: 1502. 04199] [INSPIRE].

[33] T. Han, S.K. Kang and J. Sayre, Muon $g-2$ in the aligned two Higgs doublet model, JHEP 02 (2016) 097 [arXiv: 1511.05162] [INSPIRE].

[34] A. Cherchiglia, P. Kneschke, D. Stöckinger and H. Stöckinger-Kim, The muon magnetic moment in the 2HDM: complete two-loop result, JHEP 01 (2017) 007 [Erratum ibid. 10 (2021) 242] [arXiv: 1607.06292] [INSPIRE].

[35] A. Cherchiglia, D. Stöckinger and H. Stöckinger-Kim, Muon g- 2 in the 2HDM: maximum results and detailed phenomenology, Phys. Rev. D 98 (2018) 035001 [arXiv:1711.11567] [INSPIRE].

[36] S.-P. Li, X.-Q. Li, Y.-Y. Li, Y.-D. Yang and X. Zhang, Power-aligned 2HDM: a correlative perspective on $(g-2)_{e, \mu}$, JHEP 01 (2021) 034 [arXiv: 2010.02799] [INSPIRE].

[37] P. Athron, C. Balázs, D.H. Jacob, W. Kotlarski, D. Stöckinger and H. Stöckinger-Kim, New physics explanations of $a_{\mu}$ in light of the FNAL muon $g-2$ measurement, JHEP 09 (2021) 080 [arXiv: 2104.03691] [INSPIRE].

[38] Y. Omura, E. Senaha and K. Tobe, Lepton-flavor-violating Higgs decay $h \rightarrow \mu \tau$ and muon anomalous magnetic moment in a general two Higgs doublet model, JHEP 05 (2015) 028 [arXiv: 1502.07824] [INSPIRE].

[39] A. Crivellin, J. Heeck and P. Stoffer, A perturbed lepton-specific two-Higgs-doublet model facing experimental hints for physics beyond the Standard Model, Phys. Rev. Lett. 116 (2016) 081801 [arXiv:1507.07567] [INSPIRE].

[40] S. Iguro, Y. Omura and M. Takeuchi, Testing the 2HDM explanation of the muon $g-2$ anomaly at the LHC, JHEP 11 (2019) 130 [arXiv:1907.09845] [INSPIRE].

[41] S. Jana, V.P.K. and S. Saad, Resolving electron and muon $g-2$ within the $2 H D M$, Phys. Rev. D 101 (2020) 115037 [arXiv:2003.03386] [INSPIRE].

[42] N. Ghosh and J. Lahiri, Revisiting a generalized two-Higgs-doublet model in light of the muon anomaly and lepton flavor violating decays at the HL-LHC, Phys. Rev. D 103 (2021) 055009 [arXiv: 2010.03590] [INSPIRE].

[43] W.-S. Hou, R. Jain, C. Kao, G. Kumar and T. Modak, Collider prospects for muon $g-2$ in general two Higgs doublet model, Phys. Rev. D 104 (2021) 075036 [arXiv:2105.11315] [INSPIRE].

[44] W.-S. Hou and G. Kumar, Charged lepton flavor violation in light of muon $g-2$, arXiv:2107.14114 [INSPIRE].

[45] O. Atkinson, M. Black, A. Lenz, A. Rusov and J. Wynne, Cornering the two Higgs doublet model type II, arXiv:2107.05650 [INSPIRE]. 
[46] W.-S. Hou, Decadal mission for the new physics Higgs/flavor era, arXiv:2109.02557 [INSPIRE].

[47] L. Lopez Honorez, E. Nezri, J.F. Oliver and M.H.G. Tytgat, The inert doublet model: an archetype for dark matter, JCAP 02 (2007) 028 [hep-ph/0612275] [INSPIRE].

[48] M. Gustafsson, E. Lundstrom, L. Bergstrom and J. Edsjo, Significant gamma lines from inert Higgs dark matter, Phys. Rev. Lett. 99 (2007) 041301 [astro-ph/0703512] [INSPIRE].

[49] E.M. Dolle and S. Su, The inert dark matter, Phys. Rev. D 80 (2009) 055012 [arXiv:0906.1609] [INSPIRE].

[50] L. Lopez Honorez and C.E. Yaguna, The inert doublet model of dark matter revisited, JHEP 09 (2010) 046 [arXiv: 1003.3125] [INSPIRE].

[51] L. Lopez Honorez and C.E. Yaguna, A new viable region of the inert doublet model, JCAP 01 (2011) 002 [arXiv: 1011.1411] [INSPIRE].

[52] W. Chao and M.J. Ramsey-Musolf, Hidden from view: neutrino masses, dark matter, and TeV-scale leptogenesis in a neutrinophilic two-Higgs-doublet model, Phys. Rev. D 89 (2014) 033007 [arXiv: 1212.5709] [INSPIRE].

[53] A. Goudelis, B. Herrmann and O. Stål, Dark matter in the inert doublet model after the discovery of a higgs-like boson at the LHC, JHEP 09 (2013) 106 [arXiv:1303.3010] [INSPIRE].

[54] A. Arhrib, Y.-L.S. Tsai, Q. Yuan and T.-C. Yuan, An updated analysis of inert Higgs doublet model in light of the recent results from LUX, Planck, AMS-02 and LHC, JCAP 06 (2014) 030 [arXiv:1310.0358] [INSPIRE].

[55] C. Bonilla, D. Sokolowska, N. Darvishi, J.L. Diaz-Cruz and M. Krawczyk, IDMS: inert dark matter model with a complex singlet, J. Phys. G 43 (2016) 065001 [arXiv:1412.8730] [INSPIRE].

[56] F.S. Queiroz and C.E. Yaguna, The CTA aims at the Inert Doublet Model, JCAP 02 (2016) 038 [arXiv: 1511.05967] [INSPIRE].

[57] G. Arcadi, 2HDM portal for singlet-doublet dark matter, Eur. Phys. J. C 78 (2018) 864 [arXiv: 1804.04930] [INSPIRE].

[58] Y.-L.S. Tsai, V.Q. Tran and C.-T. Lu, Confronting dark matter co-annihilation of inert two Higgs doublet model with a compressed mass spectrum, JHEP 06 (2020) 033 [arXiv: 1912.08875] [INSPIRE].

[59] D.A. Camargo, M.D. Campos, T.B. de Melo and F.S. Queiroz, A two Higgs doublet model for dark matter and neutrino masses, Phys. Lett. B 795 (2019) 319 [arXiv:1901.05476] [INSPIRE].

[60] W.-S. Hou, Tree level $t \rightarrow c h$ or $h \rightarrow t \bar{c}$ decays, Phys. Lett. B 296 (1992) 179 [inSPIRE].

[61] F. Mahmoudi and O. Stal, Flavor constraints on the two-Higgs-doublet model with general Yukawa couplings, Phys. Rev. D 81 (2010) 035016 [arXiv:0907.1791] [INSPIRE].

[62] HFLAV collaboration, Averages of b-hadron, c-hadron and $\tau$-lepton properties as of 2018, Eur. Phys. J. C 81 (2021) 226 [arXiv: 1909.12524] [InSPIRE].

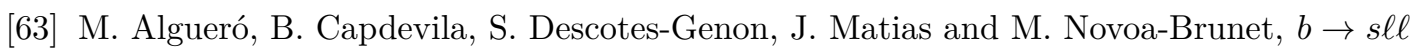
global fits after Moriond 2021 results, in $55^{\text {th }}$ Rencontres de Moriond on QCD and high energy interactions, (2021) [arXiv:2104.08921] [INSPIRE]. 
[64] T. Hurth, F. Mahmoudi, D.M. Santos and S. Neshatpour, More indications for lepton nonuniversality in $b \rightarrow s \ell^{+} \ell^{-}$, Phys. Lett. B 824 (2022) 136838 [arXiv:2104.10058] [INSPIRE].

[65] J. Bhom, M. Chrzaszcz, F. Mahmoudi, M.T. Prim, P. Scott and M. White, A model-independent analysis of $b \rightarrow s \mu^{+} \mu^{-}$transitions with GAMBIT's FlavBit, Eur. Phys. J. C 81 (2021) 1076 [arXiv: 2006. 03489] [INSPIRE].

[66] J.M. Cline, Scalar doublet models confront $\tau$ and $b$ anomalies, Phys. Rev. D 93 (2016) 075017 [arXiv: 1512.02210] [INSPIRE].

[67] J. Cardozo, J.H. Muñoz, N. Quintero and E. Rojas, Analysing the charged scalar boson contribution to the charged-current B meson anomalies, J. Phys. G 48 (2021) 035001 [arXiv: 2006.07751] [INSPIRE].

[68] P. Arnan, D. Bečirević, F. Mescia and O. Sumensari, Two Higgs doublet models and $b \rightarrow s$ exclusive decays, Eur. Phys. J. C 77 (2017) 796 [arXiv:1703.03426] [INSPIRE].

[69] A. Arhrib et al., $R_{K^{(*)}}$ anomaly in type-III $2 H D M$, arXiv:1710.05898 [INSPIRE].

[70] A. Crivellin, D. Müller and C. Wiegand, $b \rightarrow s \ell^{+} \ell^{-}$transitions in two-Higgs-doublet models, JHEP 06 (2019) 119 [arXiv:1903.10440] [INSPIRE].

[71] J. Herrero-Garcia, M. Nebot, F. Rajec, M. White and A.G. Williams, Higgs quark flavor violation: simplified models and status of general two-Higgs-doublet model, JHEP 02 (2020) 147 [arXiv: 1907.05900] [INSPIRE].

[72] Muon G-2 collaboration, Measurement of the positive muon anomalous magnetic moment to 0.46 ppm, Phys. Rev. Lett. 126 (2021) 141801 [arXiv:2104.03281] [INSPIRE].

[73] T. Aoyama et al., The anomalous magnetic moment of the muon in the Standard Model, Phys. Rept. 887 (2020) 1 [arXiv:2006.04822] [INSPIRE].

[74] M. Davier, A. Hoecker, B. Malaescu and Z. Zhang, Reevaluation of the hadronic vacuum polarisation contributions to the Standard Model predictions of the muon $g-2$ and $\alpha\left(m_{Z}^{2}\right)$ using newest hadronic cross-section data, Eur. Phys. J. C 77 (2017) 827 [arXiv: 1706. 09436] [INSPIRE].

[75] A. Keshavarzi, D. Nomura and T. Teubner, Muon $g-2$ and $\alpha\left(M_{Z}^{2}\right)$ : a new data-based analysis, Phys. Rev. D 97 (2018) 114025 [arXiv: 1802.02995] [InSPIRE].

[76] G. Colangelo, M. Hoferichter and P. Stoffer, Two-pion contribution to hadronic vacuum polarization, JHEP 02 (2019) 006 [arXiv:1810.00007] [INSPIRE].

[77] M. Hoferichter, B.-L. Hoid and B. Kubis, Three-pion contribution to hadronic vacuum polarization, JHEP 08 (2019) 137 [arXiv:1907.01556] [INSPIRE].

[78] M. Davier, A. Hoecker, B. Malaescu and Z. Zhang, A new evaluation of the hadronic vacuum polarisation contributions to the muon anomalous magnetic moment and to $\alpha\left(m_{Z}^{2}\right)$, Eur. Phys. J. C 80 (2020) 241 [Erratum ibid. 80 (2020) 410] [arXiv:1908.00921] [INSPIRE].

[79] A. Keshavarzi, D. Nomura and T. Teubner, $g-2$ of charged leptons, $\alpha\left(M_{Z}^{2}\right)$, and the hyperfine splitting of muonium, Phys. Rev. D 101 (2020) 014029 [arXiv:1911.00367] [INSPIRE]. 
[80] A. Kurz, T. Liu, P. Marquard and M. Steinhauser, Hadronic contribution to the muon anomalous magnetic moment to next-to-next-to-leading order, Phys. Lett. B 734 (2014) 144 [arXiv:1403.6400] [INSPIRE].

[81] K. Melnikov and A. Vainshtein, Hadronic light-by-light scattering contribution to the muon anomalous magnetic moment revisited, Phys. Rev. D 70 (2004) 113006 [hep-ph/0312226] [INSPIRE].

[82] P. Masjuan and P. Sánchez-Puertas, Pseudoscalar-pole contribution to the $\left(g_{\mu}-2\right): a$ rational approach, Phys. Rev. D 95 (2017) 054026 [arXiv:1701.05829] [INSPIRE].

[83] G. Colangelo, M. Hoferichter, M. Procura and P. Stoffer, Dispersion relation for hadronic light-by-light scattering: two-pion contributions, JHEP 04 (2017) 161 [arXiv:1702.07347] [INSPIRE].

[84] M. Hoferichter, B.-L. Hoid, B. Kubis, S. Leupold and S.P. Schneider, Dispersion relation for hadronic light-by-light scattering: pion pole, JHEP 10 (2018) 141 [arXiv:1808.04823] [INSPIRE].

[85] A. Gérardin, H.B. Meyer and A. Nyffeler, Lattice calculation of the pion transition form factor with $N_{f}=2+1$ Wilson quarks, Phys. Rev. D 100 (2019) 034520 [arXiv: 1903.09471] [INSPIRE].

[86] J. Bijnens, N. Hermansson-Truedsson and A. Rodríguez-Sánchez, Short-distance constraints for the HLbL contribution to the muon anomalous magnetic moment, Phys. Lett. B 798 (2019) 134994 [arXiv: 1908.03331] [INSPIRE].

[87] G. Colangelo, F. Hagelstein, M. Hoferichter, L. Laub and P. Stoffer, Longitudinal short-distance constraints for the hadronic light-by-light contribution to $(g-2)_{\mu}$ with large- $N_{c}$ Regge models, JHEP 03 (2020) 101 [arXiv:1910.13432] [INSPIRE].

[88] G. Colangelo, M. Hoferichter, A. Nyffeler, M. Passera and P. Stoffer, Remarks on higher-order hadronic corrections to the muon g-2, Phys. Lett. B 735 (2014) 90 [arXiv: 1403.7512] [INSPIRE].

[89] T. Blum et al., Hadronic light-by-light scattering contribution to the muon anomalous magnetic moment from lattice QCD, Phys. Rev. Lett. 124 (2020) 132002 [arXiv: 1911.08123] [INSPIRE].

[90] T. Aoyama, M. Hayakawa, T. Kinoshita and M. Nio, Complete tenth-order QED contribution to the muon $g-2$, Phys. Rev. Lett. 109 (2012) 111808 [arXiv:1205.5370] [INSPIRE].

[91] T. Aoyama, T. Kinoshita and M. Nio, Theory of the anomalous magnetic moment of the electron, Atoms 7 (2019) 28 [INSPIRE].

[92] A. Czarnecki, W.J. Marciano and A. Vainshtein, Refinements in electroweak contributions to the muon anomalous magnetic moment, Phys. Rev. D 67 (2003) 073006 [Erratum ibid. 73 (2006) 119901] [hep-ph/0212229] [INSPIRE].

[93] C. Gnendiger, D. Stöckinger and H. Stöckinger-Kim, The electroweak contributions to $(g-2)_{\mu}$ after the Higgs boson mass measurement, Phys. Rev. D 88 (2013) 053005 [arXiv: 1306.5546] [INSPIRE].

[94] GAMBIT collaboration, GAMBIT: the Global and Modular Beyond-the-standard-model Inference Tool, Eur. Phys. J. C 77 (2017) 784 [Addendum ibid. 78 (2018) 98] [arXiv: 1705. 07908] [INSPIRE]. 
[95] A. Kvellestad, P. Scott and M. White, GAMBIT and its application in the search for physics beyond the standard model, arXiv:1912.04079 [INSPIRE].

[96] GAMBIT collaboration, ColliderBit: a GAMBIT module for the calculation of high-energy collider observables and likelihoods, Eur. Phys. J. C 77 (2017) 795 [arXiv:1705.07919] [INSPIRE].

[97] GAMBIT Dark Matter Workgroup collaboration, DarkBit: a GAMBIT module for computing dark matter observables and likelihoods, Eur. Phys. J. C $7 \mathbf{7}$ (2017) 831 [arXiv: 1705. 07920] [INSPIRE].

[98] GAMBIT Flavour Workgroup collaboration, FlavBit: a GAMBIT module for computing flavour observables and likelihoods, Eur. Phys. J. C 77 (2017) 786 [arXiv: 1705. 07933] [INSPIRE].

[99] M. Chrzaszcz, M. Drewes, T.E. Gonzalo, J. Harz, S. Krishnamurthy and C. Weniger, A frequentist analysis of three right-handed neutrinos with GAMBIT, Eur. Phys. J. C 80 (2020) 569 [arXiv: 1908. 02302] [INSPIRE].

[100] GAMBIT Cosmology Workgroup collaboration, CosmoBit: a GAMBIT module for computing cosmological observables and likelihoods, JCAP 02 (2021) 022 [arXiv:2009.03286] [INSPIRE].

[101] GAMBIT collaboration, Global fits of GUT-scale SUSY models with GAMBIT, Eur. Phys. J. $C 77$ (2017) 824 [arXiv:1705.07935] [InSPIRE].

[102] GAMBIT collaboration, A global fit of the MSSM with GAMBIT, Eur. Phys. J. C 77 (2017) 879 [arXiv: 1705.07917] [INSPIRE].

[103] GAMBIT collaboration, Combined collider constraints on neutralinos and charginos, Eur. Phys. J. C 79 (2019) 395 [arXiv:1809.02097] [INSPIRE].

[104] GAMBIT collaboration, Status of the scalar singlet dark matter model, Eur. Phys. J. C 77 (2017) 568 [arXiv: 1705. 07931] [InSPIRE].

[105] P. Athron, J.M. Cornell, F. Kahlhoefer, J. McKay, P. Scott and S. Wild, Impact of vacuum stability, perturbativity and XENON1T on global fits of $Z_{2}$ and $Z_{3}$ scalar singlet dark matter, Eur. Phys. J. C 78 (2018) 830 [arXiv:1806.11281] [INSPIRE].

[106] GAMBIT collaboration, Global analyses of Higgs portal singlet dark matter models using GAMBIT, Eur. Phys. J. C $\mathbf{7 9}$ (2019) 38 [arXiv: 1808.10465] [InSPIRE].

[107] S. Bloor et al., The GAMBIT universal model machine: from Lagrangians to likelihoods, Eur. Phys. J. C 81 (2021) 1103 [arXiv:2107.00030] [InSPIRE].

[108] GAMBIT collaboration, Thermal WIMPs and the scale of new physics: global fits of Dirac dark matter effective field theories, Eur. Phys. J. C 81 (2021) 992 [arXiv:2106.02056] [INSPIRE].

[109] S. Hoof, F. Kahlhoefer, P. Scott, C. Weniger and M. White, Axion global fits with Peccei-Quinn symmetry breaking before inflation using GAMBIT, JHEP 03 (2019) 191 [Erratum ibid. 11 (2019) 099] [arXiv:1810.07192] [INSPIRE].

[110] P. Athron et al., Global fits of axion-like particles to XENON1T and astrophysical data, JHEP 05 (2021) 159 [arXiv: 2007.05517] [INSPIRE]. 
[111] Gambit Cosmology Workgroup collaboration, Strengthening the bound on the mass of the lightest neutrino with terrestrial and cosmological experiments, Phys. Rev. D 103 (2021) 123508 [arXiv : 2009. 03287] [inSPIRE].

[112] F. Rajec, W. Su, M. White and A.G. Williams, Exploring the $2 H D M$ with global fits in GAMBIT, EPJ Web Conf. 245 (2020) 06022 [arXiv:2007.11942] [INSPIRE].

[113] GAMBIT Models Workgroup collaboration, SpecBit, DecayBit and PrecisionBit: GAMBIT modules for computing mass spectra, particle decay rates and precision observables, Eur. Phys. J. C 78 (2018) 22 [arXiv:1705.07936] [INSPIRE].

[114] F. Mahmoudi, SuperIso: a program for calculating the isospin asymmetry of $B \rightarrow K^{*} \gamma$ in the MSSM, Comput. Phys. Commun. 178 (2008) 745 [arXiv:0710.2067] [INSPIRE].

[115] F. Mahmoudi, SuperIso v2.3: a program for calculating flavor physics observables in supersymmetry, Comput. Phys. Commun. 180 (2009) 1579 [arXiv:0808.3144] [INSPIRE].

[116] F. Mahmoudi, SuperIso v3.0, flavor physics observables calculations: extension to NMSSM, Comput. Phys. Commun. 180 (2009) 1718 [inSPIRE].

[117] S. Neshatpour and F. Mahmoudi, Flavour physics with SuperIso, PoS TOOLS2020 (2021) 036 [arXiv:2105.03428] [INSPIRE].

[118] D. Eriksson, J. Rathsman and O. Stal, 2HDMC: two-Higgs-doublet model calculator physics and manual, Comput. Phys. Commun. 181 (2010) 189 [arXiv:0902.0851] [INSPIRE].

[119] J. Bhom and M. Chrzaszcz, HEPLike: an open source framework for experimental likelihood evaluation, Comput. Phys. Commun. 254 (2020) 107235 [arXiv: 2003. 03956] [INSPIRE].

[120] GAMBIT collaboration, Comparison of statistical sampling methods with ScannerBit, the GAMBIT scanning module, Eur. Phys. J. C 77 (2017) 761 [arXiv:1705.07959] [InSPIRE].

[121] S. Davidson and H.E. Haber, Basis-independent methods for the two-Higgs-doublet model, Phys. Rev. D 72 (2005) 035004 [Erratum ibid. 72 (2005) 099902] [hep-ph/0504050] [INSPIRE].

[122] S.L. Glashow and S. Weinberg, Natural conservation laws for neutral currents, Phys. Rev. D 15 (1977) 1958 [INSPIRE].

[123] J.F. Gunion, H.E. Haber, G.L. Kane and S. Dawson, The Higgs hunter's guide, Front. Phys. 80 (2000) 1 [INSPIRE].

[124] G.W.-S. Hou, Perspectives and outlook from HEP window on the universe, Int. J. Mod. Phys. A 34 (2019) 1930002 [arXiv: 1901.04033] [INSPIRE].

[125] H.E. Haber and D. O'Neil, Basis-independent methods for the two-Higgs-doublet model III: the CP-conserving limit, custodial symmetry, and the oblique parameters $S, T, U$, Phys. Rev. D 83 (2011) 055017 [arXiv: 1011.6188] [INSPIRE].

[126] J. Hernandez-Sanchez, S. Moretti, R. Noriega-Papaqui and A. Rosado, Off-diagonal terms in Yukawa textures of the type-III 2-Higgs doublet model and light charged Higgs boson phenomenology, JHEP 07 (2013) 044 [arXiv:1212.6818] [INSPIRE].

[127] A. Crivellin, A. Kokulu and C. Greub, Flavor-phenomenology of two-Higgs-doublet models with generic Yukawa structure, Phys. Rev. D 87 (2013) 094031 [arXiv:1303.5877] [INSPIRE].

[128] J.F. Gunion and H.E. Haber, The CP conserving two Higgs doublet model: the approach to the decoupling limit, Phys. Rev. D 67 (2003) 075019 [hep-ph/0207010] [INSPIRE]. 
[129] GFitTer Group collaboration, The global electroweak fit at NNLO and prospects for the LHC and ILC, Eur. Phys. J. C $\mathbf{7 4}$ (2014) 3046 [arXiv:1407.3792] [INSPIRE].

[130] B. Altunkaynak, W.-S. Hou, C. Kao, M. Kohda and B. McCoy, Flavor changing heavy Higgs interactions at the LHC, Phys. Lett. B 751 (2015) 135 [arXiv:1506.00651] [INSPIRE].

[131] W.-S. Hou and T. Modak, Probing top changing neutral Higgs couplings at colliders, Mod. Phys. Lett. A 36 (2021) 2130006 [arXiv:2012.05735] [INSPIRE].

[132] S. Iguro and K. Tobe, $R\left(D^{(*)}\right)$ in a general two Higgs doublet model, Nucl. Phys. B 925 (2017) 560 [arXiv: 1708.06176] [inSPIRE].

[133] C. Degrande, Automatic evaluation of $U V$ and $R_{2}$ terms for beyond the standard model Lagrangians: a proof-of-principle, Comput. Phys. Commun. 197 (2015) 239 [arXiv:1406.3030] [INSPIRE].

[134] V. Shtabovenko, R. Mertig and F. Orellana, New developments in FeynCalc 9.0, Comput. Phys. Commun. 207 (2016) 432 [arXiv:1601.01167] [INSPIRE].

[135] H.H. Patel, Package-X: a mathematica package for the analytic calculation of one-loop integrals, Comput. Phys. Commun. 197 (2015) 276 [arXiv:1503.01469] [INSPIRE].

[136] F. Mahmoudi and S. Neshatpour, SuperIso v4.1: a program for calculating flavour physics observables in SM, 2HDM and supersymmetry, http://superiso.in2p3.fr, (2020).

[137] A.J. Buras, Weak Hamiltonian, CP-violation and rare decays, in Les Houches summer school in theoretical physics, session 68: probing the standard model of particle interactions, (1998), pg. 281 [hep-ph/9806471] [INSPIRE].

[138] G. Buchalla, A.J. Buras and M.E. Lautenbacher, Weak decays beyond leading logarithms, Rev. Mod. Phys. 68 (1996) 1125 [hep-ph/9512380] [INSPIRE].

[139] A. Celis, M. Jung, X.-Q. Li and A. Pich, Sensitivity to charged scalars in $B \rightarrow D^{(*)} \tau \nu_{\tau}$ and $B \rightarrow \tau \nu_{\tau}$ decays, JHEP 01 (2013) 054 [arXiv: 1210.8443] [INSPIRE].

[140] A. Crivellin, C. Greub and A. Kokulu, Explaining $B \rightarrow D \tau \nu, B \rightarrow D^{*} \tau \nu$ and $B \rightarrow \tau \nu$ in a 2HDM of type-III, Phys. Rev. D 86 (2012) 054014 [arXiv:1206.2634] [INSPIRE].

[141] R. Alonso, B. Grinstein and J. Martin Camalich, Lifetime of $B_{c}^{-}$constrains explanations for anomalies in $B \rightarrow D^{(*)} \tau \nu$, Phys. Rev. Lett. 118 (2017) 081802 [arXiv:1611.06676] [INSPIRE].

[142] S. Descotes-Genon, T. Hurth, J. Matias and J. Virto, Optimizing the basis of $B \rightarrow K^{*} l l$ observables in the full kinematic range, JHEP 05 (2013) 137 [arXiv:1303.5794] [INSPIRE].

[143] W. Altmannshofer, P. Ball, A. Bharucha, A.J. Buras, D.M. Straub and M. Wick, Symmetries and asymmetries of $B \rightarrow K^{*} \mu^{+} \mu^{-}$decays in the standard model and beyond, JHEP 01 (2009) 019 [arXiv:0811.1214] [INSPIRE].

[144] A. Czarnecki and W.J. Marciano, Electroweak radiative corrections to $b \rightarrow s \gamma$, Phys. Rev. Lett. 81 (1998) 277 [hep-ph/9804252] [INSPIRE].

[145] M. Misiak et al., Estimate of $\mathcal{B}\left(\bar{B} \rightarrow X_{s} \gamma\right)$ at $O\left(\alpha_{s}^{2}\right)$, Phys. Rev. Lett. 98 (2007) 022002 [hep-ph/0609232] [INSPIRE].

[146] M. Misiak and M. Steinhauser, NNLO QCD corrections to the $\bar{B} \rightarrow X_{s} \gamma$ matrix elements using interpolation in $m_{c}$, Nucl. Phys. B 764 (2007) 62 [hep-ph/0609241] [INSPIRE]. 
[147] M. Czakon, P. Fiedler, T. Huber, M. Misiak, T. Schutzmeier and M. Steinhauser, The $\left(Q_{7}, Q_{1,2}\right)$ contribution to $\bar{B} \rightarrow X_{s} \gamma$ at $\mathcal{O}\left(\alpha_{\mathrm{s}}^{2}\right)$, JHEP 04 (2015) 168 [arXiv:1503.01791] [INSPIRE].

[148] M. Misiak and M. Steinhauser, Weak radiative decays of the $B$ meson and bounds on $M_{H^{ \pm}}$ in the two-Higgs-doublet model, Eur. Phys. J. C 77 (2017) 201 [arXiv:1702.04571] [INSPIRE].

[149] M. Misiak, A. Rehman and M. Steinhauser, Towards $\bar{B} \rightarrow X_{s} \gamma$ at the NNLO in $Q C D$ without interpolation in $m_{c}$, JHEP 06 (2020) 175 [arXiv:2002.01548] [INSPIRE].

[150] LHCb collaboration, Test of lepton universality in beauty-quark decays, arXiv:2103.11769 [INSPIRE].

[151] LHCb collaboration, Measurement of the $B_{s}^{0} \rightarrow \mu^{+} \mu^{-}$decay properties and search for the $B^{0} \rightarrow \mu^{+} \mu^{-}$and $B_{s}^{0} \rightarrow \mu^{+} \mu^{-} \gamma$ decays, arXiv:2108.09283 [INSPIRE].

[152] LHCb collaboration, Test of lepton universality with $B^{0} \rightarrow K^{* 0} \ell^{+} \ell^{-}$decays, JHEP 08 (2017) 055 [arXiv: 1705. 05802] [INSPIRE].

[153] LHCb collaboration, Combination of the ATLAS, CMS and LHCb results on the $B_{(s)}^{0} \rightarrow \mu^{+} \mu^{-}$decays, Tech. Rep. LHCb-CONF-2020-002, CERN, Geneva, Switzerland (2020).

[154] Particle Data Group collaboration, Review of particle physics, PTEP 2020 (2020) 083C01 [INSPIRE].

[155] Belle collaboration, Search for $B \rightarrow h \nu \bar{\nu}$ decays with semileptonic tagging at Belle, Phys. Rev. D 96 (2017) 091101 [Addendum ibid. 97 (2018) 099902] [arXiv:1702.03224] [INSPIRE].

[156] J. Bhom and M. Chrząszcz, HEPLikeData, https://github.com/mchrzasz/HEPLikeData, (2020).

[157] BeLLE collaboration, Measurement of the CKM matrix element $\left|V_{c b}\right|$ from $B^{0} \rightarrow D^{*-} \ell^{+} \nu_{\ell}$ at Belle, Phys. Rev. D 100 (2019) 052007 [Erratum ibid. 103 (2021) 079901] [arXiv: 1809.03290] [INSPIRE].

[158] C. Murgui, A. Peñuelas, M. Jung and A. Pich, Global fit to $b \rightarrow c \tau \nu$ transitions, JHEP 09 (2019) 103 [arXiv: 1904.09311] [InSPIRE].

[159] M. Tanaka and R. Watanabe, New physics in the weak interaction of $\bar{B} \rightarrow D^{(*)} \tau \bar{\nu}$, Phys. Rev. D 87 (2013) 034028 [arXiv: 1212.1878] [INSPIRE].

[160] M. Beneke and G. Buchalla, The B $B_{c}$ meson lifetime, Phys. Rev. D 53 (1996) 4991 [hep-ph/9601249] [INSPIRE].

[161] A.G. Akeroyd and C.-H. Chen, Constraint on the branching ratio of $B_{c} \rightarrow \tau \bar{\nu}$ from LEP1 and consequences for $R\left(D^{(*)}\right)$ anomaly, Phys. Rev. D 96 (2017) 075011 [arXiv: 1708.04072] [INSPIRE].

[162] M. Blanke et al., Impact of polarization observables and $B_{c} \rightarrow \tau \nu$ on new physics explanations of the $b \rightarrow c \tau \nu$ anomaly, Phys. Rev. D 99 (2019) 075006 [arXiv:1811.09603] [INSPIRE].

[163] J. Aebischer and B. Grinstein, A novel determination of the $B_{c}$ lifetime, arXiv:2108.10285 [INSPIRE]. 
[164] J. Aebischer and B. Grinstein, Standard model prediction of the $B_{c}$ lifetime, arXiv:2105.02988 [INSPIRE].

[165] LHCb collaboration, Measurement of the ratio of branching fractions $\mathcal{B}\left(B_{c}^{+} \rightarrow J / \psi \tau^{+} \nu_{\tau}\right) / \mathcal{B}\left(B_{c}^{+} \rightarrow J / \psi \mu^{+} \nu_{\mu}\right)$, Phys. Rev. Lett. 120 (2018) 121801 [arXiv: 1711.05623] [INSPIRE].

[166] Belle collaboration, Measurement of the $D^{*-}$ polarization in the decay $B^{0} \rightarrow D^{*-} \tau^{+} \nu_{\tau}$, in $10^{\text {th }}$ International workshop on the CKM unitarity triangle, (2019) [arXiv:1903.03102] [INSPIRE].

[167] S. Bhattacharya, S. Nandi and S. Kumar Patra, $b \rightarrow c \tau \nu_{\tau}$ decays: a catalogue to compare, constrain, and correlate new physics effects, Eur. Phys. J. C 79 (2019) 268 [arXiv: 1805.08222] [INSPIRE].

[168] BABAR collaboration, Measurement of an excess of $\bar{B} \rightarrow D^{(*)} \tau^{-} \bar{\nu}_{\tau}$ decays and implications for charged Higgs bosons, Phys. Rev. D 88 (2013) 072012 [arXiv:1303.0571] [INSPIRE].

[169] Fermilab Lattice and MILC collaborations, $B_{(s)}^{0}$-mixing matrix elements from lattice QCD for the standard model and beyond, Phys. Rev. D 93 (2016) 113016 [arXiv: 1602.03560] [INSPIRE].

[170] D.M. Straub, flavio: a python package for flavour and precision phenomenology in the standard model and beyond, arXiv:1810.08132 [INSPIRE].

[171] M. Jung, A. Pich and P. Tuzon, Charged-Higgs phenomenology in the aligned two-Higgs-doublet model, JHEP 11 (2010) 003 [arXiv: 1006.0470] [INSPIRE].

[172] Heavy Flavor Averaging Group collaboration, Averages of b-hadron and c-hadron properties at the end of 2007, arXiv:0808.1297 [INSPIRE].

[173] A.G. Akeroyd and F. Mahmoudi, Constraints on charged Higgs bosons from $D_{s}^{ \pm} \rightarrow \mu^{ \pm} \nu$ and $D_{s}^{ \pm} \rightarrow \tau^{ \pm} \nu$, JHEP 04 (2009) 121 [arXiv:0902.2393] [INSPIRE].

[174] Particle Data Group collaboration, Review of particle physics, PTEP 2020 (2020) 083C01 [INSPIRE].

[175] Y. Omura, E. Senaha and K. Tobe, $\tau$ - and $\mu$-physics in a general two Higgs doublet model with $\mu-\tau$ flavor violation, Phys. Rev. D 94 (2016) 055019 [arXiv: 1511.08880] [INSPIRE].

[176] W.-S. Hou and G. Kumar, Muon flavor violation in two Higgs doublet model with extra Yukawa couplings, Phys. Rev. D 102 (2020) 115017 [arXiv:2008.08469] [InSPIRE].

[177] Y. Kuno and Y. Okada, Muon decay and physics beyond the standard model, Rev. Mod. Phys. 73 (2001) 151 [hep-ph/9909265] [INSPIRE].

[178] CMS collaboration, Search for lepton-flavor violating decays of the Higgs boson in the $\mu \tau$ and eT final states in proton-proton collisions at $\sqrt{s}=13 \mathrm{TeV}$, Phys. Rev. D 104 (2021) 032013 [arXiv: 2105.03007] [INSPIRE].

[179] S. Bifani, S. Descotes-Genon, A. Romero Vidal and M.-H. Schune, Review of lepton universality tests in B decays, J. Phys. G 46 (2019) 023001 [arXiv:1809.06229] [INSPIRE].

[180] S.S. AbdusSalam et al., Simple and statistically sound strategies for analysing physical theories, arXiv:2012.09874 [INSPIRE].

[181] P. Scott, Pippi - painless parsing, post-processing and plotting of posterior and likelihood samples, Eur. Phys. J. Plus 127 (2012) 138 [arXiv:1206.2245] [INSPIRE]. 
[182] A. Arbey, F. Mahmoudi, O. Stal and T. Stefaniak, Status of the charged Higgs boson in two Higgs doublet models, Eur. Phys. J. C 78 (2018) 182 [arXiv:1706.07414] [InSPIRE].

[183] A. Crivellin, J. Heeck and D. Müller, Large $h \rightarrow b s$ in generic two-Higgs-doublet models, Phys. Rev. D 97 (2018) 035008 [arXiv:1710.04663] [InSPIRE].

[184] A. Wahab El Kaffas, P. Osland and O.M. Ogreid, Constraining the two-Higgs-doublet-model parameter space, Phys. Rev. D 76 (2007) 095001 [arXiv:0706. 2997] [INSPIRE].

[185] A. Arhrib, R. Benbrik, C.-H. Chen, R. Guedes and R. Santos, Double neutral Higgs production in the two-Higgs doublet model at the LHC, JHEP 08 (2009) 035 [arXiv: 0906.0387] [INSPIRE].

[186] CMS collaboration, Measurement of the $P_{1}$ and $P_{5}^{\prime}$ angular parameters of the decay $B^{0} \rightarrow K^{* 0} \mu^{+} \mu^{-}$in proton-proton collisions at $\sqrt{s}=8 \mathrm{TeV}$, Tech. Rep.

CMS-PAS-BPH-15-008, CERN, Geneva, Switzerland (2017).

[187] ATLAS collaboration, Angular analysis of $B_{d}^{0} \rightarrow K^{*} \mu^{+} \mu^{-}$decays in pp collisions at $\sqrt{s}=8 \mathrm{TeV}$ with the ATLAS detector, JHEP 10 (2018) 047 [arXiv:1805. 04000] [INSPIRE].

[188] LHCb collaboration, Measurement of CP-averaged observables in the $B^{0} \rightarrow K^{* 0} \mu^{+} \mu^{-}$ decay, Phys. Rev. Lett. 125 (2020) 011802 [arXiv:2003.04831] [InSPIRE].

[189] LHCb collaboration, Measurements of the S-wave fraction in $B^{0} \rightarrow K^{+} \pi^{-} \mu^{+} \mu^{-}$decays and the $B^{0} \rightarrow K^{*}(892)^{0} \mu^{+} \mu^{-}$differential branching fraction, JHEP 11 (2016) 047 [Erratum ibid. 04 (2017) 142] [arXiv: 1606.04731] [INSPIRE].

[190] LHCb collaboration, Differential branching fraction and angular analysis of the $B^{+} \rightarrow K^{+} \mu^{+} \mu^{-}$decay, JHEP 02 (2013) 105 [arXiv: 1209.4284] [InSPIRE].

[191] LHCb collaboration, Differential branching fractions and isospin asymmetries of $B \rightarrow K^{(*)} \mu^{+} \mu^{-}$decays, JHEP 06 (2014) 133 [arXiv:1403.8044] [INSPIRE].

[192] LHCb collaboration, Differential branching fraction and angular analysis of $\Lambda_{b}^{0} \rightarrow \Lambda \mu^{+} \mu^{-}$ decays, JHEP 06 (2015) 115 [Erratum ibid. 09 (2018) 145] [arXiv:1503.07138] [InSPIRE].

[193] W. Detmold and S. Meinel, $\Lambda_{b} \rightarrow \Lambda \ell^{+} \ell^{-}$form factors, differential branching fraction, and angular observables from lattice QCD with relativistic b quarks, Phys. Rev. D 93 (2016) 074501 [arXiv: 1602.01399] [INSPIRE].

[194] LHCb collaboration, Angular analysis and differential branching fraction of the decay $B_{s}^{0} \rightarrow \phi \mu^{+} \mu^{-}$, JHEP 09 (2015) 179 [arXiv:1506.08777] [INSPIRE].

[195] LHCb collaboration, Branching fraction measurements of the rare $B_{s}^{0} \rightarrow \phi \mu^{+} \mu^{-}$and $B_{s}^{0} \rightarrow f_{2}^{\prime}(1525) \mu^{+} \mu^{-}$decays, Phys. Rev. Lett. 127 (2021) 151801 [arXiv:2105.14007] [INSPIRE].

[196] LHCb collaboration, Search for lepton-universality violation in $B^{+} \rightarrow K^{+} \ell^{+} \ell^{-}$decays, Phys. Rev. Lett. 122 (2019) 191801 [arXiv:1903.09252] [INSPIRE].

[197] BeLLe collaboration, Test of lepton-flavor universality in $B \rightarrow K^{*} \ell^{+} \ell^{-}$decays at Belle, Phys. Rev. Lett. 126 (2021) 161801 [arXiv: 1904. 02440] [INSPIRE].

[198] BELLE collaboration, Test of lepton flavor universality and search for lepton flavor violation in $B \rightarrow$ Kll decays, JHEP 03 (2021) 105 [arXiv: 1908.01848] [INSPIRE].

[199] ATLAS collaboration, Search for top-quark decays $t \rightarrow H q$ with $36 \mathrm{fb}^{-1}$ of $p p$ collision data at $\sqrt{s}=13 \mathrm{TeV}$ with the ATLAS detector, JHEP 05 (2019) 123 [arXiv:1812.11568] [INSPIRE]. 
[200] D. Barducci and A.J. Helmboldt, Quark flavour-violating Higgs decays at the ILC, JHEP 12 (2017) 105 [arXiv:1710.06657] [INSPIRE].

[201] BeLle-II collaboration, The Belle II physics book, PTEP 2019 (2019) 123C01 [Erratum ibid. 2020 (2020) 029201] [arXiv: 1808.10567] [INSPIRE].

[202] W.-S. Hou and G. Kumar, Coming decade of $h \rightarrow \tau \mu$ and $\tau \rightarrow \mu \gamma$ interplay in $\tau$ flavor violation search, Phys. Rev. D 101 (2020) 095017 [arXiv: 2003.03827] [INSPIRE].

[203] LHCb collaboration, Physics case for an LHCb upgrade II - opportunities in flavour physics, and beyond, in the HL-LHC era, arXiv:1808.08865 [INSPIRE].

[204] A.I. Davydychev and J.B. Tausk, Two loop selfenergy diagrams with different masses and the momentum expansion, Nucl. Phys. B 397 (1993) 123 [inSPIRE].

[205] B. Capdevila, U. Laa and G. Valencia, Anatomy of a six-parameter fit to the $b \rightarrow s \ell^{+} \ell^{-}$ anomalies, Eur. Phys. J. C 79 (2019) 462 [arXiv:1811.10793] [INSPIRE]. 